Development of surface micromachined Aluminum Nitride airbridges for piezoelectric MEMS/NEMS applications by Metal Organic Vapor Phase Epitaxy techniques

\author{
Sridhar Kuchibhatla \\ West Virginia University
}

Follow this and additional works at: https://researchrepository.wvu.edu/etd

\author{
Recommended Citation \\ Kuchibhatla, Sridhar, "Development of surface micromachined Aluminum Nitride air-bridges for \\ piezoelectric MEMS/NEMS applications by Metal Organic Vapor Phase Epitaxy techniques" (2011). \\ Graduate Theses, Dissertations, and Problem Reports. 4743. \\ https://researchrepository.wvu.edu/etd/4743
}

This Dissertation is protected by copyright and/or related rights. It has been brought to you by the The Research Repository @ WVU with permission from the rights-holder(s). You are free to use this Dissertation in any way that is permitted by the copyright and related rights legislation that applies to your use. For other uses you must obtain permission from the rights-holder(s) directly, unless additional rights are indicated by a Creative Commons license in the record and/ or on the work itself. This Dissertation has been accepted for inclusion in WVU Graduate Theses, Dissertations, and Problem Reports collection by an authorized administrator of The Research Repository @ WVU. For more information, please contact researchrepository@mail.wvu.edu. 


\title{
Development of surface micromachined Aluminum Nitride air-bridges for piezoelectric MEMS/NEMS applications by Metal Organic Vapor Phase Epitaxy techniques
}

\author{
Sridhar Kuchibhatla \\ Dissertation \\ Submitted to the \\ College of Engineering and Mineral Resources \\ at West Virginia University \\ in partial fulfillment of the requirements \\ for the degree of
}

\author{
Doctor of Philosophy \\ in \\ Electrical Engineering \\ Dr. Dimitris Korakakis, Chair \\ Dr.Xian-An Cao \\ Dr.Parviz Famouri \\ Dr.Lawrence A. Hornak \\ Dr.Nianqiang Wu \\ Department of Electrical Engineering \\ Morgantown, West Virginia \\ 2011
}

Keywords: Aluminum Nitride, Micro-FTIR, Piezoelectric, MEMS/NEMS 


\title{
Abstract \\ Development of surface micromachined Aluminum Nitride air-bridges for piezoelectric MEMS/NEMS applications by Metal Organic Vapor Phase Epitaxy techniques
}

\author{
Sridhar Kuchibhatla
}

Group III-nitrides have attracted considerable attention for piezoelectric Micro/Nano electromechanical (MEMS/NEMS) applications due to their excellent bio compatibility, well developed growth techniques for high quality thin films and structural stability at high temperatures when compared to the commonly used piezoelectric metal oxides. Among the group III-nitrides Aluminum Nitride (AIN) possess superior material properties such as highest piezoelectric coefficient and good mechanical properties. Growth techniques for fabricating group III-nitride MEMS/NEMS by metal organic vapor phase epitaxy (MOVPE) techniques have involved sacrificial layers such as epitaxial group IIInitrides/alloys, nanocrystalline films and porous interlayers. However, the material properties of the MOVPE grown films on the amorphous sacrificial layers such as silicon oxide have not been adequately investigated to evaluate potential MEMS/NEMS devices such as piezoelectric micro/ nanofluidic channels.

This work demonstrates a process for the fabrication of Aluminum nitride (AlN) thin film air-bridges using MOVPE techniques on silicon templates. Micro-FTIR techniques were used to study the crystallographic orientation of the AIN thin film airbridges with lateral dimensions as low as $100 \mu \mathrm{m}$. FTIR results also show that the wet etching process to remove the underlying sacrificial layer also improves the material properties of the AlN films on $\mathrm{SiO}_{\mathrm{x}}$. The study indicates that AlN air-bridges are polycrystalline in nature and are preferentially c-axis oriented after wet etching. Lateral field excitation of the piezoelectric films and laser Doppler vibrometer techniques were combined to investigate the piezoelectric response of the AIN films on the sacrificial layer. Lateral field excitation of the AlN films grown on the amorphous sacrificial layer shows that the AlN films exhibit piezoelectric properties. The displacement of the AIN air-bridges obtained by lateral field actuation is around $1 \mathrm{~nm}$ over an air-gap of $130 \mathrm{~nm}$ after the removal of the sacrificial layer. However, the mismatch in the coefficient of thermal expansion between the substrate and thin films induces significant residual stress in the heterostructure. The AlN air-bridges on silicon substrate exhibit fracture due to the tensile residual stress exceeding the fracture limit. 


\section{Acknowledgements}

I would like to take this opportunity to thank my advisor Dr. Dimitris Korakakis for his guidance and support. I would also like to thank my committee members . Dr.Xian-An Cao, Dr.Parviz Famouri, Dr.Lawrence A. Hornak, Dr.Nianqiang Wu for their time and support. Dr. Kolin Brown and the microfabrication facility staff for the clean room training. Finally I would like to thank all the current and former students of the lab for the help and encouragement. 


\section{Table of Contents:}

Title page ..............................................................................................................................

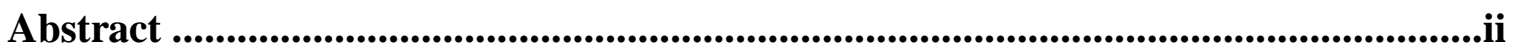

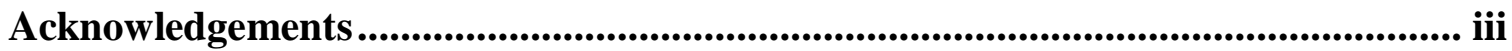

Table of Contents: ....................................................................................................................iv

Table of Figures/tables ......................................................................................................................vi

Chapter 1. Introduction.......................................................................................................1

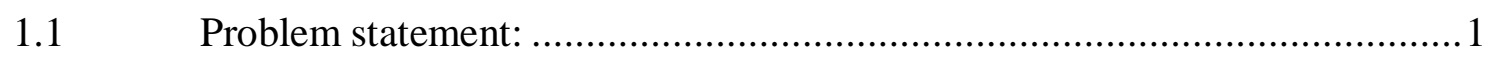

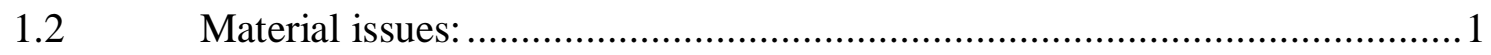

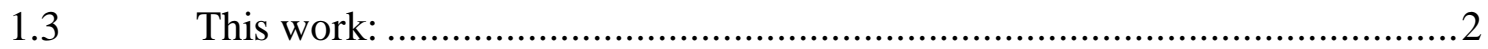

Chapter 2. Literature Review..............................................................................................3

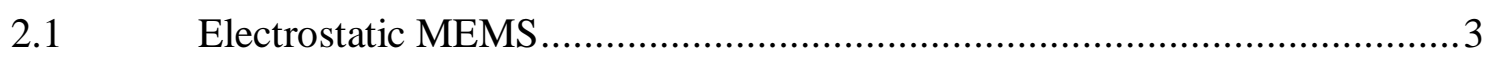

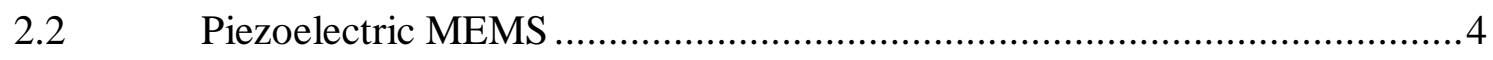

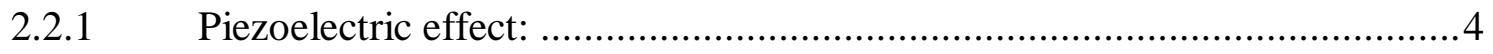

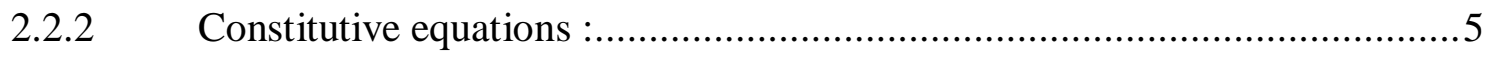

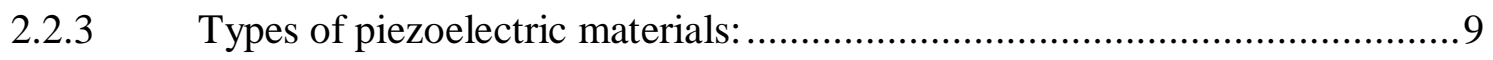

$2.3 \quad$ Introduction to III nitride MEMS .........................................................

2.3.1 Strategies to fabricate MEMS devices:...................................................12

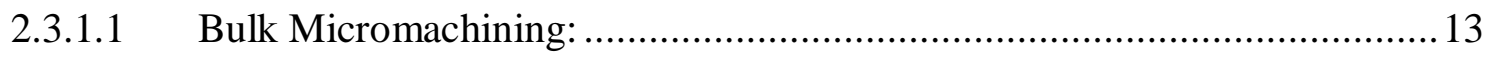

2.3.1.2 Surface Micromachining:.................................................................. 14

2.3.1.2.1 Types of sacrificial layers deposition and etching techniques: ....................16

2.3.2 Lateral growth techniques III nitrides:......................................................2 21

Chapter 3. Experimental Method ..................................................................................24

3.1 Flow process for fabricating AlN air-bridges: .....................................24

3.2 Growth conditions for AlN films: .....................................................24

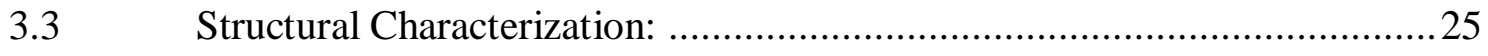




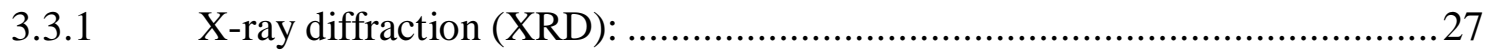

3.3.2 Micro-Raman spectroscopy: ...................................................... 27

3.3.2.1 Raman spectroscopy: .......................................................... 28

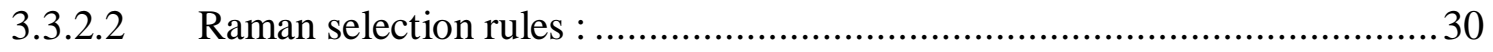

3.3.2.3 Raman scattering under polarized incident light .................................. 32

3.3.2.4 Lattice vibrations: ................................................................... 34

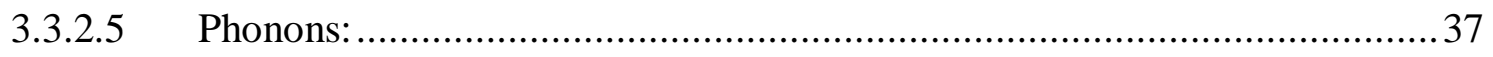

3.3.2.6 Zone center phonons of wurtzite Aluminum Nitride (AlN) ......................38

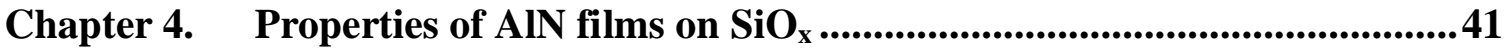

4.1 Determination of crystal orientation by polarized Micro-Raman

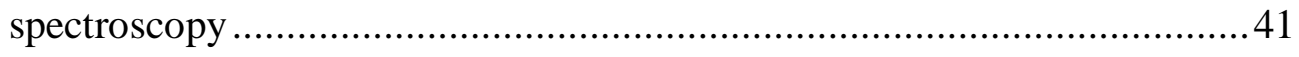

4.2 Etch rate of AIN in AZ 400K solution and crystal quality: .......................43

4.3 Identifying crystallographic orientation by Micro-FTIR techniques: .........45

4.3.1 Investigating the effect of buffered HF on AlN Micro-FTIR ....................51

4.4 Profile of $\mathrm{SiO}_{\mathrm{x}}$ sacrificial layer after etching in buffered $\mathrm{HF}$....................55

4.5 Effect of buffered HF on AlN films grown on $\mathrm{SiO}_{\mathrm{x}}-$ Micro Raman...........57

4.5.1 Hydrolysis of AlN in aqueous solutions: ....................................... 62

4.6 Residual stress in AlN air-bridges due to thermal mismatch: ....................64

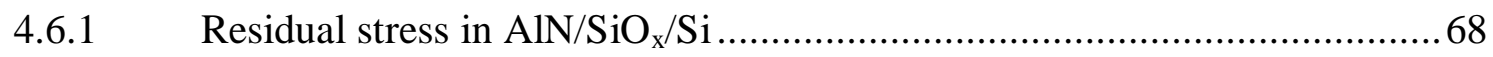

4.6.2 Residual stress in $\mathrm{AlN} / \mathrm{SiO}_{\mathrm{x}} / \mathrm{GaN} /$ Sapphire ......................................69

Chapter 5. Piezoelectric characterization of AIN films on $\mathrm{SiO}_{\mathrm{x}}$............................71

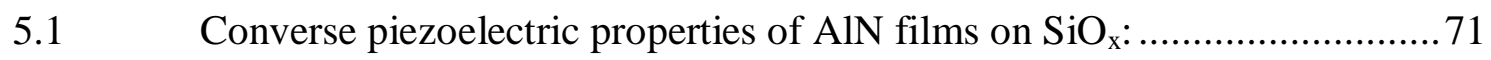

5.1.1 Measurement of longitudinal piezoelectric coefficient of AlN films by laser

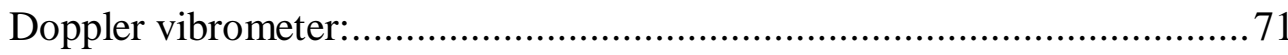


5.1.2 Measurement of longitudinal piezoelectric coefficient of AlN films by laser

Doppler vibrometer:

5.1.3 Piezoelectric response of AlN films on $\mathrm{SiO}_{\mathrm{x}}$ measured by laser Doppler

vibrometer:

5.1.4 Piezoelectric actuation of AlN air-bridges..................................... 84

5.1.5 Fabrication of AlN air-bridges with electrodes .................................... 86

Chapter 6. Conclusions and future work .............................................................91

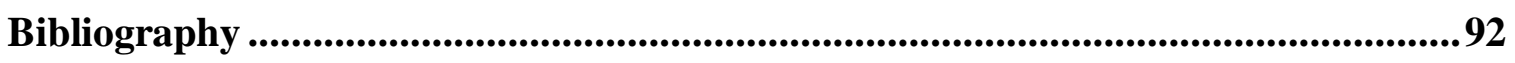

Appendix..........................................................................................................98

Table of Figures/tables

Figure 2.1: Electrostatic actuated parallel electrodes separated by an air-gap.

Figure 2.2: Molecular model explaining the piezoelectric effect (a) Undisturbed molecule

(b) Molecule subjected to external mechanical stress (c) Polarized material 4

Figure 2.3: Change in the dimensions of the piezoelectric material due time varying voltages.

Figure 2.4: Piezoelectric cube showing the coordinate axis in three-dimensional analysis

Figure 2.5: (a) Randomly aligned electric dipoles (b) and (c) Poling process

Figure 2.6: Common configuration of piezoelectric actuated beams (a) Bimorph cantilever (b) Doubly clamped beam

Figure 2.7: Commonly used strategies for fabricating suspended MEMS/NEMS structures .

Figure 2.8: Bulk micromachining process.

Figure 2.9: All-nitride MEMS heterostructure .14

Figure 2.10: Surface micromachining process.

Figure 2.11: Variation of emission wavelength and bandgap of III-nitrides with Al, Ga and In mole fraction 
Figure 2.12: Etch rate of AlN films grown by different methods

Figure 2.13: Process flow to fabricate GaN cantilever on sapphire substrate by PEC (a) masking from front side (b) masking from backside with $\mathrm{GaN}$ as light absorbing filter

Figure 2.14: Schematic of doping selective PEC etch process for fabrication of GaN microfluidic channels

Figure 2.15: Lateral growth process of GaN (a) Schematic (b) SEM cross section image

Figure 2.16: Lateral growth process of AlGaN (a) Schematic (b) SEM cross section image of $\mathrm{AlGaN}$ growth on $\mathrm{SiO}_{\mathrm{x}}$ with $40 \%$ aluminum

Figure 3.1: Flow process for the fabrication of thin film AlN air-bridge.

Figure 3.2: (a) Wurtzite crystallographic structure of AlN (b) Lattice points on the c plane .25

Figure 3.3: Atomic arrangement at the $\mathrm{AlN}(0001) / \mathrm{Si}(111)$ .27

Figure 3.4: Micro-Raman spectrometer .28

Figure 3.5: Raman scattering effect .29

Figure 3.6: Illustration of Raman .29

Figure 3.7: (a) Stokes shift (b) Anti-Stokes shift 30

Figure 3.8: Dipole induced in a molecule due to external electric field..... .31

Figure 3.9: Polarization direction of Raman scattered with the direction of polarizability of the molecule not parallel to the source polarization direction .33

Figure 3.10: Lattice structure of $\mathrm{NaCl}$ 34

Figure 3.11: A one dimensional linear chain with a diatomic lattice .35

Figure 3.12: Dispersion relation of $\omega_{-}$and $\omega_{+}$with respect to wave number $k$ in the first Brillouin zone of a linear chain of atoms . .36 
Figure 3.13: (a) Transverse optical (b) Longitudinal optical lattice vibrations modes in a diatomic unit cell

Figure 3.14: (a) Unit cell of AlN crystal [58] (b) Zone center optical phonons in a wurtzite crystal .38

Figure 3.15: Optical phonons in a wurtzite crystal .39

Figure 4.1: Flow process for the fabrication of an AlN thin film air-bridge. 41

Figure 4.2: Raman spectra of as-grown AlN film on $\mathrm{SiO}_{x}$ (a) GaN/Sapphire.

Figure 4.3: SEM images of as-grown AlN films on (a) GaN (b) Si (111) templates patterned with $\mathrm{SiO}_{\mathrm{x}}$

Figure 4.4: SEM cross-section of AlN films on (a) GaN (b) Si (111) after wet etching in AZ $400 \mathrm{~K}$ for 20 minutes .43

Figure 4.5: SEM surface image of $\mathrm{AlN}$ on $\mathrm{SiO}_{\mathrm{x}}$ and $\mathrm{Si}$ (111) after wet etching in $\mathrm{AZ400K}$

Figure 4.6: Infrared transmission spectra of a polar optical crystal in the Restrahelen band for thick films. .45

Figure 4.7 : Infrared transmission spectra of a polar optical crystal in the Restrahelen band for thin films.

Figure 4.8: (a) Micro-FTIR transmission measurement setup [Varian Inc] (b) Oblique angle of incidence on the infrared rays in a micro-FTIR .47

Figure 4.9: Process flow for the fabrication of an AIN thin film suspended structure. ....48

Figure 4.10: SEM images of the AlN film grown on Si (111) substrate patterned with $\mathrm{SiO}_{\mathrm{x}}$ stripes: (a) Surface (b) Cross-section. .49

Figure 4.11: FTIR transmission spectrum of the AlN film on $\mathrm{Si}(111)$ and $\mathrm{SiO}_{\mathrm{x}}$ .50

Figure 4.12: Variation in the FTIR transmission spectrum of $\mathrm{AlN} / \mathrm{SiO}_{\mathrm{x}}$ bilayer measured after etching the samples for different times in buffered $\operatorname{HF}(10: 1)$ solution.51

Figure 4.13: SEM surface images of AlN film on $\mathrm{SiO}_{\mathrm{x}}$ : (a) As-grown (b) Etched in buffered HF (10:1) for 3 hours. 
Figure 4.14: SEM cross section image of a suspended AlN structure after sacrificial layer etching.

Figure 4.15: FTIR transmission spectrum of as-grown AlN film on $\mathrm{SiO}_{\mathrm{x}}$. Dashed lines show deconvoluted Lorentz functions.

Figure 4.16: Ratio of the integrated area of the vibrational modes of etched samples with respect to unetched samples for $\mathrm{AlN}$ film on $\mathrm{SiO}_{\mathrm{x}}$

Figure 4.17: Process flow for investigating the etch profile of $\mathrm{SiO}_{\mathrm{x}}$ .55

Figure 4.18: Etch profile of $\mathrm{SiOx}$ after removal of photo resistmask .56

Figure 4.19: Optical images of the AIN bridges after etching in buffered HF (10:1) solution for $30 \mathrm{~min}$ (a) $\mathrm{Si}$ (111) (b) GaN.

Figure 4.20: Raman spectra collected from the discolored regions of AlN bridges after etching in buffered HF solution (10:1) showing the AIN E2 (high) peak (a) GaN (b) Si (111).

Figure 4.21: SEM cross-section image of an AIN air-bridge after sacrificial layer etching.

Figure 4.22: AlN air-bridge $80 \mu \mathrm{m}$ wide on GaN (a) Raman spectra after peak separation (b) Direction of line scan indicated by red arrow from left to right (c) peak position of E2 (high) mode along the line scan. .59

Figure 4.23: AlN air-bridge $80 \mu \mathrm{m}$ wide on Si (111) (a) Raman spectra after peak separation (b) Direction of line scan indicated by red arrow from left to right (c) peak position of E2 (high) mode along the line scan. .60

Figure 4.24: Texture growth model for thin films on amorphous substrates 62

Figure 4.25: Schematic diagram of oxidation state of aluminum nitride thin film

Figure 4.26: Optical images of full eteched AlN air bridges on Si (111) and GaN/Sapphire template 64

Figure 4.27: Examples of intrinsic and extrinsic stress in thin films .65 
Figure 4.28: Residual stress in thin film due to mismatch of the coefficient of thermal expansion between the film and the substrate

Figure 4.29: Peak position of the E1 (TO) mode of $\mathrm{AlN}$ films on $\mathrm{SiO}_{\mathrm{x}}$ as a function of etching time

Figure 4.30: Phone chord buckling delamination pattern 69

Figure 5.1: (a) Schematic of wurtzite crystal with electric field applied along the c-axis (b) Modified constitutive equations

Figure 5.2: Measurement of the thickness change of a piezoelectric film by laser Doppler vibrometer .73

Figure 5.3: (a) Piezoelectric measurement of the AlN films between $\mathrm{SiO}_{\mathrm{x}}$ stripes with platinum back and top contacts (b) Apparent piezoelectric coefficient of AlN films grown on silicon substrate.

Figure 5.4: Typical thin film substrate configuration showing the influence of substrate on $\mathrm{d}_{33}$ measurement

Figure 5.5: (a) Schematic of wurtzite crystal with electric field applied normal to the caxis (b) Modified constitutive equations.

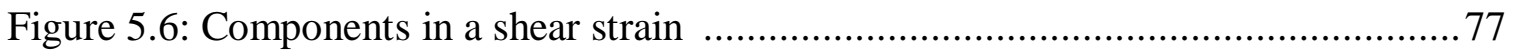

Figure 5.7: (a) Thickness shear strain (c) Length shear strain ..................................78

Figure 5.8: Electric field generated in a piezoelectric film with electrode gaps (a) $1 \mu \mathrm{m}$ (b) $10 \mu \mathrm{m}$. Modified from

Figure 5.9: Activation of thickness shear strain in AlN films through lateral field excitation .79

Figure 5.10: Piezoelectric response of AlN films actuated by lateral field excitation ......80

Figure 5.11: Displacement of top electrode for a laterally actuated AlN films grown on silicon. 81

Figure 5.12: (a) Longitudinal field actuation (b) Lateral field actuation of $\mathrm{AlN} / \mathrm{SiO}_{\mathrm{x}}$ with a platinum back and top electrode. 
Figure 5.13: Comparison of piezoelectric response of AlN films grown on Silicon substrate and $\mathrm{SiO}_{\mathrm{x}}$ sacrificial layer

Figure 5.14: (a) Longitudinal field actuation (b) Lateral field actuation of AlN air-bridge with a platinum back and top electrode.

Figure 5.15: Shear mode actuated piezoelectric membrane .85

Figure 5.16: Optical images of AlN films (a) $500 \mathrm{~nm}$ (b) $700 \mathrm{~nm}$ grown on $\mathrm{SiO}_{\mathrm{x}}$ after etching for 6.5 hours

Figure 5.17: Displacement of the AIN air-bridges observed of delaminated contacts after actuated by longitudinal field excitation.

Figure 5.18: Optical images of the AlN air-bridge after re-sputtering platinum contacts on the bridges .88

Figure 5.19: Displacement of the AlN air-bridge in the (a) lateral field actuation (b) Longitudinal field actuation configuration.

Figure 5.20: Slope of the (a) Lateral field actuated AlN air-bridges (b) Longitudinal field actuated AlN air-bridges. 90

Table 2.1. Piezoelectric coefficients and Curie temperature of commonly used materials

Table 3.1. Properties of AlN film grown on different substrates.................................26

Table 3.2: Raman configuration of allowed modes in wurtzite crystals ......................39

Table 4.1: Infrared activity of wurtzite crystals ................................................. 47

Table 4.2: FWHM values of E2 (high) peaks of AlN air-bridges.............................61

Table 4.3: Mean thermal expansion coefficients of materials used to fabricate AlN airbridges

Table 4.4: Residual stress in $\mathrm{SiO}_{\mathrm{x}}$ on various substrates at room temperature.

Table 4.5: Residual stress in AlN films on $\mathrm{SiO}_{\mathrm{x}}$ at room temperature .70

Table 5.1: Apparent $d_{33}$ value of the AlN film grown on silicon substrate .74 
Table 5.2: Slope of displacement of top electrode actuated by lateral field techniques ...81 


\section{Chapter 1. Introduction}

\subsection{Problem statement:}

To investigate the material properties of AlN thin films grown by metal organic vapor phase epitaxy techniques on amorphous sacrificial for evaluating potential MEMS/NEMS devices.

\subsection{Material issues:}

Wide bandgap group III-Nitride materials such as Aluminum Nitride (AlN) and its alloys have attracted considerable attention for the fabrication of Micro/Nano electromechanical systems (MEMS/NEMS) devices due to their excellent bio compatibility, well developed growth techniques for high quality thin films, and chemical stability at high temperatures in comparison to other common piezoelectric materials such as quartz, polyvinylidelflouride and metal oxides [1]. Among the group III-Nitrides, wurtzite AIN exhibits excellent piezoelectric properties and is expected to maintain its properties up to $1150{ }^{\circ} \mathrm{C}$ [2]. Even though the piezoelectric properties depend on the microstructure of the films, polycrystalline AIN films with preferred c-axis orientation are known to exhibit properties similar to crystalline AlN films [3], [4], [5], [6], [7]. Typically, reactive sputtering techniques have been used to deposit polycrystalline AIN films on a variety of sacrificial layers such as amorphous silicon oxide $\left(\mathrm{SiO}_{\mathrm{x}}\right)$ to fabricate free standing bridges [8]. However, the sputtered AIN system requires an underlying mechanical support structure such as silicon nitride $\left(\mathrm{Si}_{3} \mathrm{~N}_{4}\right)$ due to the low mechanical strength of the sputtered AIN films [9]. In the Metal Organic Vapor Phase Epitaxy (MOVPE) of certain III-Nitride materials, dielectric layers, such as $\mathrm{SiO}_{\mathrm{x}}$ or $\mathrm{Si}_{3} \mathrm{~N}_{4}$, can be used as a mask to promote selective growth as seen in the Selective Area Growth (SAG) or Epitaxial Lateral Overgrowth of GaN [10]. However, the high sticking coefficient and short diffusion length of Al species during MOVPE growth results in the nucleation of $\mathrm{Al}$ containing alloys on dielectric layers [11], [12]. Specifically, the growth of $\mathrm{Al}_{\mathrm{x}} \mathrm{Ga}_{1-\mathrm{x}} \mathrm{N}$ $(0<\mathrm{x}<1)$ on $\mathrm{SiO}_{\mathrm{x}}$ using MOVPE techniques has been reported in several experiments related to SAG techniques on Silicon and GaN/sapphire templates [13], [14], [15], [16], 
[17] . Growth or deposition of active piezoelectric MEMS/NEMS layer on sacrificial material is a well developed process for Si. However, the material properties of the MOVPE grown films on the amorphous sacrificial layers such as $\mathrm{SiO}_{\mathrm{x}}$ have not been thoroughly investigated to evaluate potential MEMS/NEMS devices such as piezoelectric micro/nanofluidic channels [18].

\subsection{This work:}

This work discusses the material properties of AlN thin films grown on patterned amorphous sacrificial layer on various substrates such as Si (111), sapphire. Chapter 2 discusses the literature relevant to theory of piezoelectricity and techniques to fabricate suspended structures on micromachineable and non micromachienable substrates. Chapter 3 details the process flow to fabricate AlN air-bridges addresses techniques such as Micro-Raman and Micro-FTIR to investigate the material properties of AlN thin films. In Chapter 4 we discuss the properties of $\mathrm{AlN}$ films grown on $\mathrm{SiO}_{\mathrm{x}}$. Chapter 5 discusses the actuation mechanism for the AlN air-bridges and the techniques to determine the piezoelectric coefficient of AlN. 


\section{Chapter 2. Literature Review}

Micro electro mechanical (MEMS) are a group of microsensors and actuators that can sense and react to changes in external environment through micro circuit control.

\subsection{Electrostatic MEMS}

Typically electrostatic actuation has been used to operate MEMS devices.

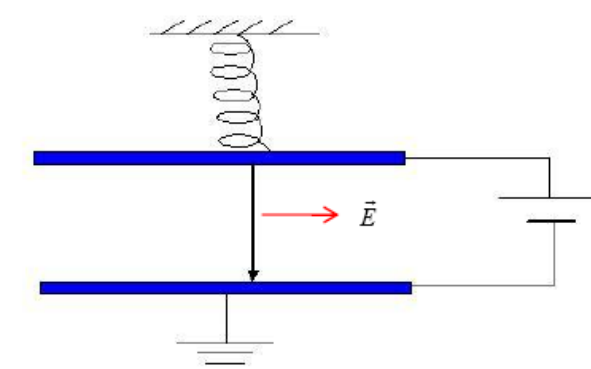

Figure 2.1: Electrostatic actuated parallel electrodes separated by an air-gap.

Shown in Figure 2.1 is a highly simplified model of an electrostatic actuator consisting of two parallel metal electrodes separated by an air-gap. Usually the lower electrode is fixed and the top electrode is free to move. Applying an electric field across the electrodes results in charges of opposite polarity on the electrodes. Oppositely charged electrodes are attracted towards each other resulting in the movement of the top electrode towards the fixed electrode thereby reducing the air-gap. To prevent an electrical short circuit between the metal electrodes and for increasing the force of attraction between the electrodes, dielectric materials such as silicon nitride, silicon-dioxide are included in-between the metal electrodes [19]. Some of the advantages of electrostatic MEMS include low power consumption, fast and simple process requirements for integrated circuit integration. Disadvantage of the electrostatic based MEMS arise from the high operating voltages which typically range between $20-80 \mathrm{~V}$ [20]. Separate on-chip power supplies or charge pumps are required to generate the high voltages. In order to improve the functionality and performance of MEMS devices, such 
as obtaining a low operating voltage, piezoelectric material systems have been synthesized and implemented in MEMS devices to realize smart MEMS devices [21].

\subsection{Piezoelectric MEMS}

\subsubsection{Piezoelectric effect:}

Piezoelectricity is a property of certain class of materials which produce charges on applying an external mechanical stress. Shown in Figure 2 is a 2D molecular model explaining piezoelectric effect [22]. Consider a molecule in which the dipoles are arranged symmetrically as shown in Figure 2.2 (a).
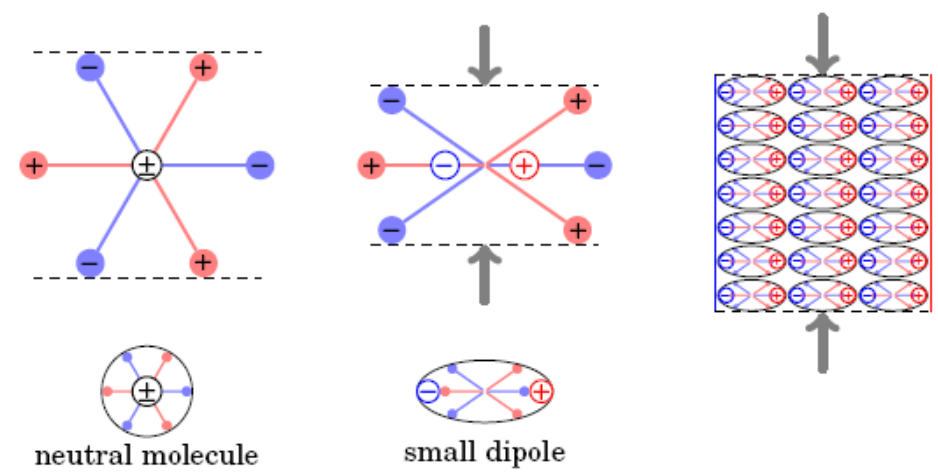

Figure 2.2: Molecular model explaining the piezoelectric effect (a) Undisturbed molecule (b) Molecule subjected to external mechanical stress (c) Polarized material [22]

Due to the symmetrical arrangement of the dipoles the electric fields cancel resulting in the positive and negative centers coinciding; as such the molecule remains electrically neutral. The positive and negative centers separate when a mechanical stress is applied externally. This leads to a net induced internal electric field as shown in Figure 2.2 (b) and the molecule is polarized. A piezoelectric material consisting of several such molecules arranged in a regular fashion and bounded by metal electrodes, when subjected to an external mechanical stress generates a net non-zero internal electric field as seen from Figure 2.2 (c). The internal electric field induces charges of opposite polarity on the electrodes. This effect is commonly referred to as a direct piezoelectric effect. The 
effect is reversible, in other words on applying an external electric field to a piezoelectric material the dipoles change length resulting in a mechanical strain and thereby a change in the dimensions of the material as shown in Figure 2.3. This is called the converse or the inverse piezoelectric effect.

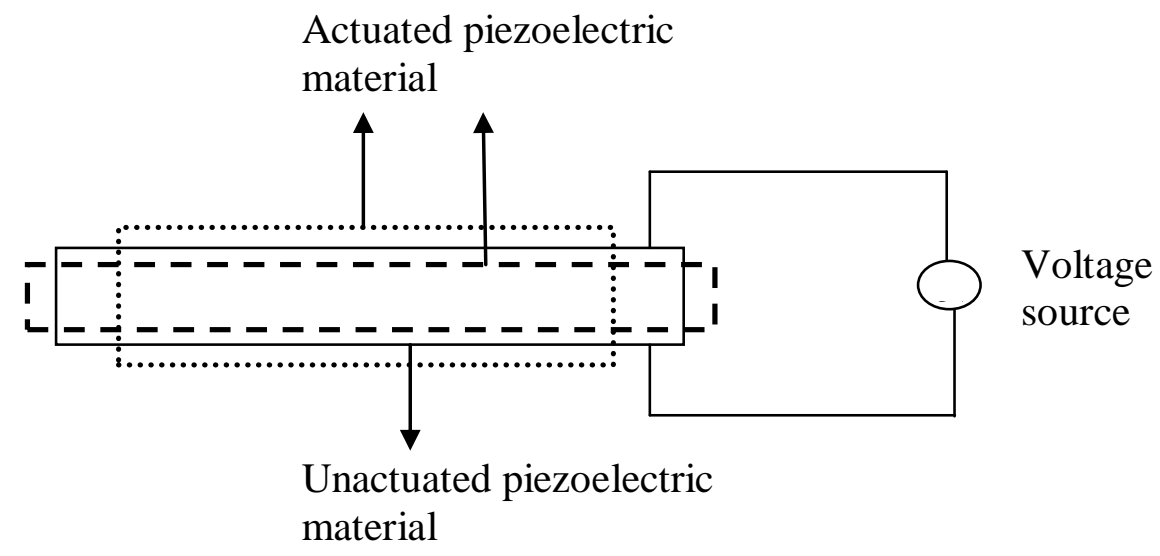

Figure 2.3: Change in the dimensions of the piezoelectric material due time varying voltages.

Harmonic mechanical vibrations can be generated by applying an AC electric field across the thickness of piezoelectric material. The AC field induces compression and expansion of the piezoelectric material alternatively, depending on the polarity of the applied voltage.

\subsubsection{Constitutive equations [23]:}

\section{Direct piezoelectric effect:}

Applying a stress to an elastic material produces an elongation or compression in the direction of the applied stress. Within the elastic limits the stress ' $\mathrm{T}$ ' is proportional to the strain produced ' $\mathrm{S}$ ' expressed mathematically as:

$$
T=Y_{S}
$$

$\mathrm{T}$ - Stress applied $\left(\mathrm{N} / \mathrm{m}^{2}\right)$

Y - Young's modulus of the material $\left(\mathrm{N} / \mathrm{m}^{2}\right)$ 
$\mathrm{S}$ - Strain produced $(\mathrm{m} / \mathrm{m})$

When a piezoelectric material is subjected to a mechanical stress in addition to an elongation or compression similar to the elastic material a charge is also produced at the electrodes at the ends of the specimen. This charge flow is produced by the motion of the dipoles in the piezoelectric material. This can be expressed mathematically as:

$$
D=d T
$$

D - Electric displacement $\left(\mathrm{C} / \mathrm{m}^{2}\right)$

d - Piezoelectric strain coefficient $(\mathrm{C} / \mathrm{N})$

$\mathrm{T}-$ Stress applied $\left(\mathrm{N} / \mathrm{m}^{2}\right)$

\section{Converse piezoelectric effect:}

On applying a constant potential across the electrodes of a piezoelectric material an electric field is produced. The electric field produces an attraction between applied charge and the dipoles resulting in displacement current. At sufficiently low electric fields the relation between the electric field and displacement can be expressed as:

$$
D=\varepsilon E
$$

D - Electric displacement $\left(\mathrm{C} / \mathrm{m}^{2}\right)$

$\epsilon$ - Dielectric permittivity $(\mathrm{F} / \mathrm{m})$

E - Applied electric field (V/m)

The attraction between the charge and dipoles leads to a change in length of the dipoles resulting in a strain in the material. In the linear regime the relation can be expressed as :

$$
s=d E
$$

$\mathrm{S}$ - Strain produced $(\mathrm{m} / \mathrm{m})$

$\mathrm{d}$ - Piezoelectric strain coefficient $(\mathrm{m} / \mathrm{V})$

E - Applied electric field (V/m)

The electromechanical effects describing the basic properties of a piezoelectric material can be expressed together in a mathematical know as the constitutive equations: 


$$
\left[\begin{array}{l}
s \\
D
\end{array}\right]=\left[\begin{array}{ll}
c & d \\
d & \varepsilon
\end{array}\right]\left[\begin{array}{l}
T \\
E
\end{array}\right]
$$

The electromechanical coupling is represented by the off diagonal term. A larger value of " $d$ " produces a higher strain for the same applied electric field. Therefore the piezoelectric coefficient is used as a parameter for comparing the piezoelectric strength of different materials.

In a three - dimensional case as shown in Figure 2.4 the properties of a material can vary with the direction vector.

There are three directions in which the electric field can be applied. These directions are labeled are $E_{n}$, where $n=1,2,3$.

Similarly there are three directions in which electric displacement can be produced and are labeled as $\mathrm{D}_{\mathrm{m}}$, where $\mathrm{m}=1,2,3$.

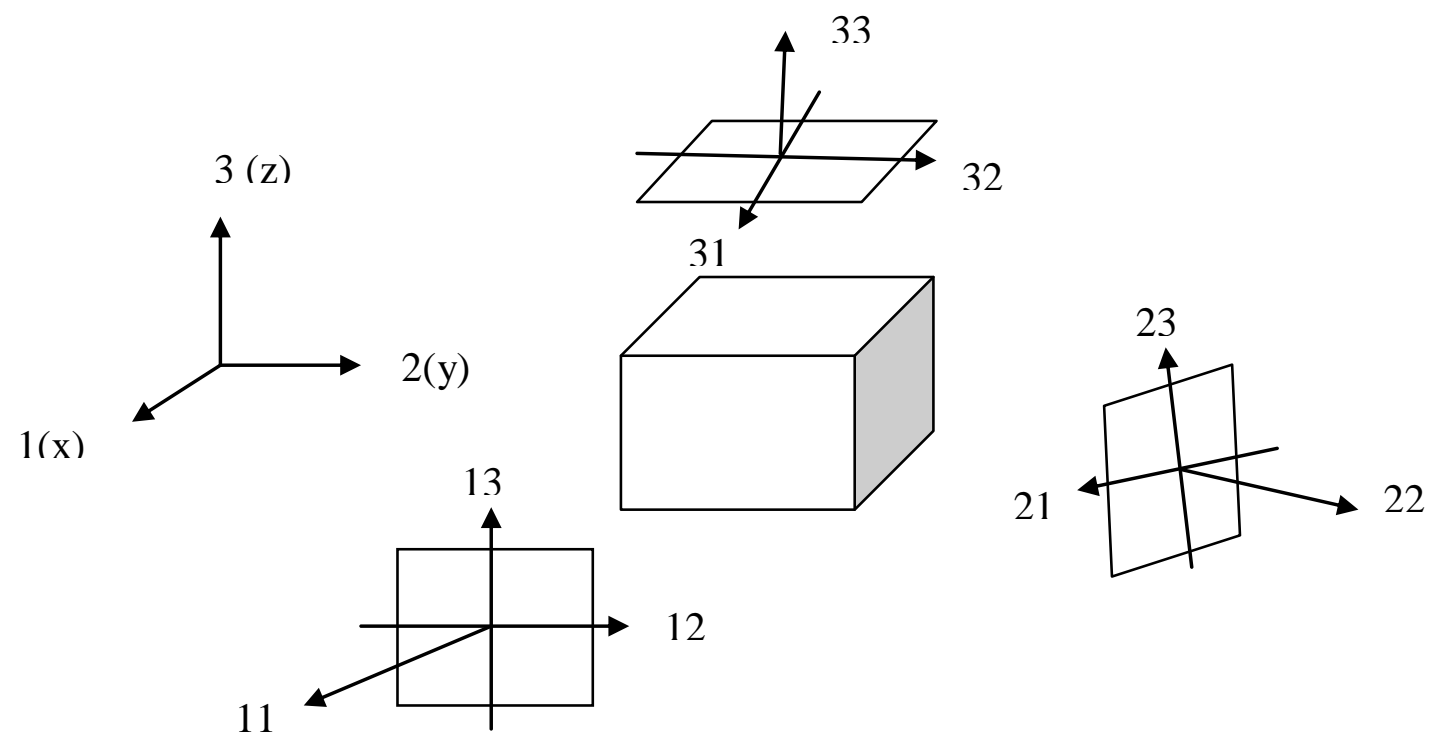

Figure 2.4: Piezoelectric cube showing the coordinate axis in three-dimensional analysis [23]. 
The strain components are labeled as $\varepsilon_{m n}$, where $\mathrm{m}, \mathrm{n}=1,2,3$. On each face of the cube three strain components can be defined. One strain component is perpendicular to the face and the remaining two are parallel to the surface of the face. The first index refers to the face upon which the strain component acts and the second subscript represents the component directions.

The general relationship between the electric displacement and electric field is now given by:

$$
\begin{array}{ll}
D_{1}=\varepsilon_{11}^{T} E_{1}+\varepsilon_{12}^{T} E_{2}+\varepsilon_{13}^{T} E_{3} & \text { Eq } 2.6(\mathrm{a}) \\
D_{1}=\varepsilon_{21}^{T} E_{1}+\varepsilon_{22}^{T} E_{2}+\varepsilon_{23}^{T} E_{3} & \text { Eq } 2.7(\mathrm{~b}) \\
D_{1}=\varepsilon_{31}^{T} E_{1}+\varepsilon_{32}^{T} E_{2}+\varepsilon_{33}^{T} E_{3} & \text { Eq } 2.8(\mathrm{c})
\end{array}
$$

In tensor form the above equations can be expressed as:

$$
D_{m}=\varepsilon_{m n}^{T} E_{n}
$$

Similarly the stress strain relation for a linear elastic material in tensor form is given by:

$$
s_{i j}=c_{i j k l}^{E} T_{k l}
$$

The nine states of strain are related to the three applied electric field through tensor form as follows:

$$
s_{i j}=d_{i j n} E_{n}
$$

The electric displacement can be related to the mechanical stress through:

$$
D_{m}=d_{m k l} T_{k l}
$$

Finally the constitutive equations for a linear piezoelectric material is expressed in the form of tensors as follows: 
Eq 2.13(a)

Eq 2.14 (b)

Where the superscripts indicate the boundary conditions under which the measurements were taken

$\mathrm{c}$ - Compliance

E - At constant electric field

$\mathrm{T}-$ At constant stress

$\mathrm{i}, \mathrm{j}, \mathrm{k}, \mathrm{l}, \mathrm{m}, \mathrm{n}=1,2,3$

\section{Compact notation for piezoelectric constitutive equations:}

The complex tensor forms can be further simplified by taking into account the symmetry. From the symmetry of the stress and strain tensors the constitutive equations can be written as:

$$
\begin{aligned}
& T_{i j}=T_{j i} \\
& S_{i j}=S_{j i}
\end{aligned}
$$

Following the above representation new stress and strain components can be defined.

$$
\begin{gathered}
\mathrm{S}_{11}=\mathrm{S}_{1}, \\
\mathrm{~S}_{22}=\mathrm{S}_{2} \\
\mathrm{~S}_{33}=\mathrm{S}_{3} \mathrm{~S}_{4}=\mathrm{S}_{23}+\mathrm{S}_{32} \\
\mathrm{~S}_{5}=\mathrm{S}_{31}+\mathrm{S}_{13} \\
\mathrm{~S}_{6}=\mathrm{S}_{12}+\mathrm{S}_{21}
\end{gathered}
$$

Eq $2.21(\mathrm{e})$

\subsubsection{Types of piezoelectric materials:}

Piezoelectric materials belong to a class of crystalline solids where the atoms are arranged in a periodic fashion throughout the bulk material. Piezoelectric materials occur naturally in the world. Examples of naturally occurring piezoelectric crystals are quartz, Rochelle salt etc. In order to increase the electromechanical coupling synthetic piezoelectric material systems have been realized which have a significantly higher $d$ value when compared to naturally occurring crystals. 


\section{Poling process:}

Generally the synthetically processed materials do not exhibit piezoelectricity due to the randomly oriented electric dipoles as shown in Figure 2.5 (a). In order to align the dipoles in a regular fashion a process called poling is performed.

a

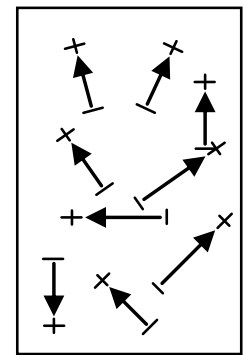

b

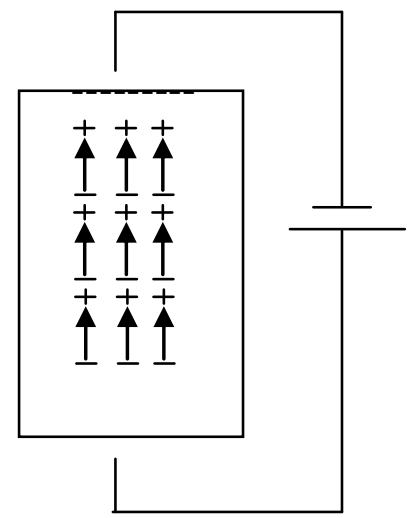

$\mathrm{c}$

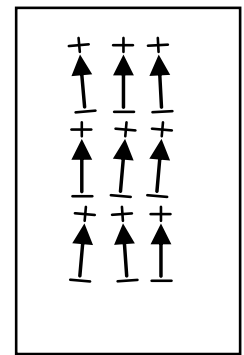

Figure 2.5: (a) Randomly aligned electric dipoles (b) and (c) Poling process [23]

In this process the material is heated to a high temperature typically above its Curie temperature Figure 2.5 (b). At this temperature the dipoles are easy to align as the material is softer. The alignment of the dipoles is achieved by applying an external electric field. The electric field produces an alignment of the dipoles along the direction of the electric field. Upon reducing the temperature quickly and removing the external electric field results in a material where the dipoles are oriented along a preferential axis as shown in Figure 2.5 (c). This axis is called the poling axis [23]. When working in the Cartesian co-ordinate system the z-axis (3) is usually taken along the poling axis.

\section{Spontaneously polarized piezoelectric crystals:}

There are certain classes of synthetic crystals which possess a net non-zero internal electric field after crystal growth without the necessity of the poling process. Such crystals are said to be spontaneously polarized. Aluminum Nitride (AlN), Gallium Nitride $(\mathrm{GaN})$, Zinc oxide $(\mathrm{ZnO})$ are some examples of spontaneously polarized piezoelectric materials. 


\section{Mode of operation of piezoelectric devices:}

Piezoelectric devices are typically designed to operate in either $d_{33}$ (thickness mode) or $d_{31}$ (transverse mode). In the $d_{33}$ mode an external electric field is applied along the polarization direction (3) and the strain produced along the polarization axis is used for the device operation. In the $\mathrm{d}_{31}$ mode of operation the external electric field is applied in the direction of polarization (3) and the strain produced in the direction perpendicular the poling axis i.e "1" or " 2 " is utilized to generate motions such as bending.

\subsection{Introduction to III nitride MEMS}

Of late wide band gap piezoelectric group III-nitrides (AlInGa-N) have attracted considerable attention for MEMS/NEMS applications. Compared to metal oxides wide bandgap materials have excellent bio compatibility, well developed growth techniques for high quality thin films and stability at high temperatures [1]. In addition metal oxide systems are not compatible with Si technology and degrade electronic circuits through $\mathrm{Zn}$ and $\mathrm{Pb}$ contamination. Unlike wide band gap semiconductors such as $\mathrm{GaN}, \mathrm{AlN}$ thin film deposition techniques for metal oxides are not well developed. Therefore metal oxide systems are difficult to integrate with NEMS. Among the group III-nitrides AIN has largest piezoelectric coefficient with $\mathrm{d}_{33}$ varying from $4-7 \mathrm{pm} / \mathrm{V}$ and possesses good mechanical strength. Thus, AlN films are suitable building blocks for fabricating electromechanical devices in micro and nanometer scale.

Commonly used piezoelectric materials and the piezoelectric coefficients are shown in Table 2.1. Curie temperature $\left(\mathrm{T}_{\mathrm{C}}\left({ }^{\circ} \mathrm{C}\right)\right)$ is the temperature at which the material loses its piezoelectric properties. Even though the piezoelectric coefficients of AlN are 
Table 2.1. Piezoelectric coefficients and Curie temperature of commonly used materials [1].

\begin{tabular}{lllll}
\hline Material & \multicolumn{3}{c}{ Piezoelectric coefficients $\left(\mathrm{pm} \mathrm{V}^{-1}\right)$} & $T_{C}\left({ }^{\circ} \mathrm{C}\right)$ \\
\hline Quartz, $\mathrm{SiO}_{2}$ & $d_{11}=2.3$ & $d_{14}=-0.67$ & & 570 \\
$\mathrm{ZnO}$ & $d_{33}=12.3$ & $d_{31}=-5.1$ & $d_{15}=-8.3$ & - \\
$\mathrm{PbTi}_{x} \mathrm{Zr}_{1-x} \mathrm{O}_{3}$ PZT-5A & $d_{33}=374$ & $d_{31}=-171$ & $d_{15}=584$ & 365 \\
$\mathrm{PbTi}_{x} \mathrm{Zr}_{1-x} \mathrm{O}_{3} \mathrm{PZT}-4$ & $d_{33}=289$ & $d_{31}=-123$ & $d_{15}=496$ & 328 \\
$\mathrm{BaZiO}_{3}$ & $d_{33}=82$ & $d_{31}=-33$ & $d_{15}=150$ & 120 \\
$\mathrm{BaPbTiO}_{3}$ & $d_{33}=120$ & $d_{31}=-50$ & $d_{15}=0.9$ & 150 \\
Polyvinylidenfluoride, PVDF & $d_{33}=27$ & $d_{31}=20$ & $d_{32}=0.9$ & 80 \\
\hline
\end{tabular}

lower than most of the materials listed in Table 1, AlN is expected to maintain its piezoelectric properties upto $1150{ }^{\circ} \mathrm{C}$ [24].

Bimorph cantilever and doubly clamped beam are some of the typical configurations of piezoelectric devices are shown in Figure 2.6.

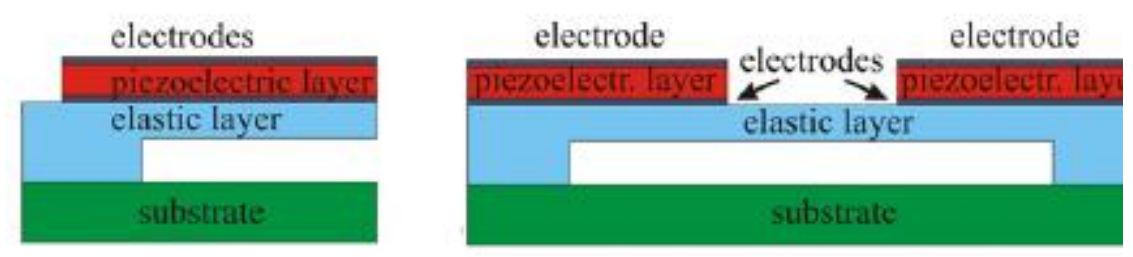

Figure 2.6: Common configuration of piezoelectric actuated beams (a) Bimorph cantilever (b) Doubly clamped beam [1].

\subsubsection{Strategies to fabricate MEMS devices:}

Some of the commonly used strategies to fabricate wide bandgap piezoelectric actuators are show in Figure 2.7. These are broadly classified into bulk micromachining and surface micromachining. 


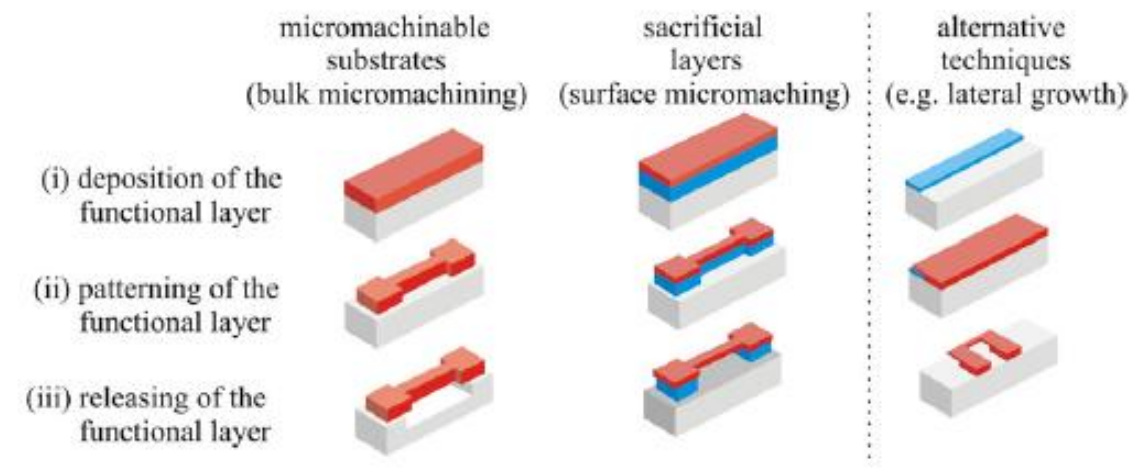

Figure 2.7: Commonly used strategies for fabricating suspended MEMS/NEMS structures [1].

\subsubsection{Bulk Micromachining:}

Bulk micromachining is the process of etching material from the bulk substrate to form suspended micro structures. As shown in Figure 2.7 in the first step a piezoelectric layer also called the functional layer is grown on the substrate. In the next step the functional layer is patterned to get the desired design. In the final step the patterned sample is subjected to a wet etching process to etch the underlying bulk substrate. Wet etching is the process of selective dissolution of materials in liquid chemicals. The wet etching process leads to the removal of the bulk material under the functional layer. This process leads to the formation of a suspended micro structure. An alternate technique to fabricate suspend structures is shown in Figure 2.8. The functional layer grown on top of the substrate is used as the etch stop layer while the substrate is etched from the rear side. Additionally the rear side is masked with etch resistant materials to form a cavity in the substrate after etching.
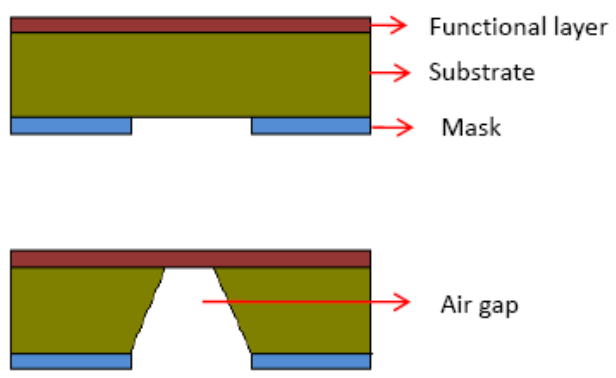

Figure 2.8: Bulk micromachining process. 
However, this technique has been developed for $\mathrm{Si}$ and $\mathrm{SiC}$ substrates only. Typically group III nitride thin films are grown on bulk sapphire substrate. The growth technique is called homoepitaxy if a crystalline functional film is grown on the substrate of the same material. The growth is referred to as heteroepitaxy if the crystalline functional layer and substrate are different materials. But techniques to selectively etch sapphire have not been developed, as such bulk micromachining techniques cannot be used to realize allnitride heteroepitaxial MEMS structures. An example of an all-nitride MEMS structure is shown in Figure 2.9.

But the advantage of sapphire is its chemical stability at high temperatures and is optically transparent. Group III-nitride thin film heterostructres grown on Si substrate result in films with high defect density. The defects degrade the material properties of the films and thereby the performance of the device. With SiC being a very expensive substrate sapphire is the only available choice for realizing all-nitride MEMS/ NEMS devices.

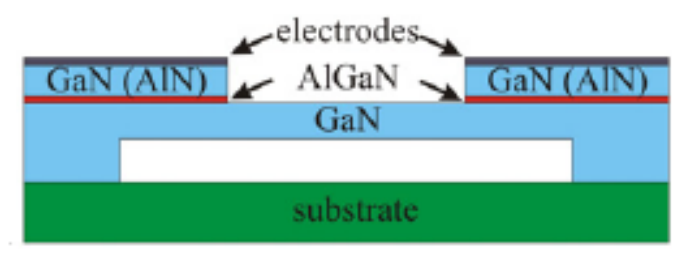

Figure 2.9: All-nitride MEMS heterostructure [25].

\subsubsection{Surface Micromachining:}

Surface micromachining is the process of selective removal of a sacrificial layer from underneath the functional layer to create suspended microstructures. As shown in Figure 2.10 a sacrificial layer is grown on the substrate. A functional layer is later grown on the sacrificial layer. In the next step the functional layer and sacrificial layer are patterned according to the design requirements. The patterned sample is subjected to wet etching. The wet etching process selectively under etches the sacrificial layer relative to the substrate and the functional layer. This results in the formation of a suspended 
microstructure. An alternate process for fabricating surface micromachined structures is shown in Figure 2.10.

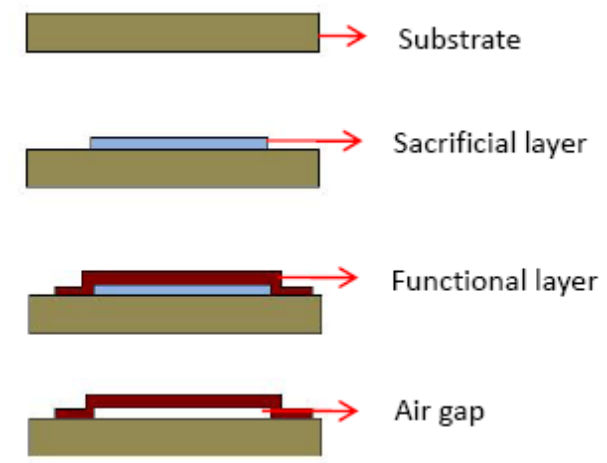

Figure 2.10: Surface micromachining process.

In this process the substrate is patterned with a sacrificial layer. In the next step a functional layer is grown on the patterned substrate. Finally the underlying sacrificial layer is selectively removed by wet etching.

Advantages of surface micromachining technique [26]:

- Ability to control the air-gap height down to few monolayers in heteroepitaxial systems.

- A wide variety of substrates and sacrificial layers can be used including sapphire substrates.

- Precise control of the undercut of the functional layers by dimensions of the sacrificial layer.

- Possibility of using epitaxial layers as sacrificial layer, which implies a high quality functional layers and thereby better device performance.

Surface micromachining is the only available technique to fabricate MEMS/NEMS structures on non micro machineable substrates such as sapphire. Consequently for realizing all-nitride heteroepitaxial MEMS devices on sapphire substrates selective etching techniques are required. 


\section{Epitaxial functional layers:}

Group III-nitride thin films can be deposited on a variety of substrates owing to the various techniques by which the films can be deposited. Techniques range from low temperature such as reactive sputtering to high temperature techniques such as metal organic vapor phase epitaxy (MOCVD or MOVPE), Molecular beam epitaxy (MBE) [7]. MOCVD and MBE are the generally preferred techniques to grow epitaxial group IIInitride thin films. Thin films deposited by reactive sputtering techniques at room temperature usually result in polycrystalline films. Epitaxial films are generally preferred for MEMS devices due to their excellent optical, mechanical and electrical properties when compared to polycrystalline films.

\subsection{Types of sacrificial layers deposition and etching techniques:}

The functional layers can be deposited on a range of sacrificial layers. The choice of the sacrificial layer depends on the technique to remove the sacrificial layer after the deposition of the functional layer. The sacrificial layer also controls the crystal quality of the functional layer. The sacrificial layers can be broadly classified into the following list:

- Epitaxial layers corresponding to group III-nitrides.

- Porous substrate or thin film.

- Nanocrysatlline thin films.

- Oxides, metals, polymers.

Oxides and polymers are usually used as sacrificial layer for the deposition amorphous and polycrystalline thin films.

\section{Epitaxial layers:}

High quality functional layers can be obtained by overgrowing the functional layers on single crystal sacrificial layers. In group III-nitrides the sacrificial layer is a combination of $\mathrm{Al}, \mathrm{In}, \mathrm{Ga}$ elements in different proportions. By varying the mole fraction of $\mathrm{Al}, \mathrm{Ga}$ or In the properties of the material system such as the lattice constant can be varied as shown in Figure 2.11. Photoelectrochemical (PEC) chemical etching and wet etching AlN are some well developed techniques to etch sacrificial group III-nitrides. 


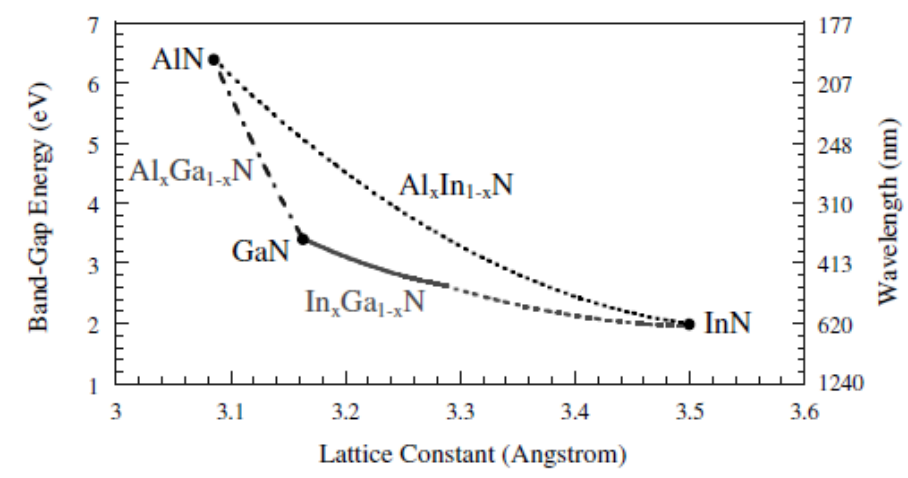

Figure 2.11: Variation of emission wavelength and bandgap of III-nitrides with Al, Ga and In mole fraction [27].

\section{Wet chemical etching:}

Due to the high chemical stability of group III-nitride films it is difficult to etch the films in corrosive solutions. GaN can be wet etched in molten salts such as $\mathrm{KOH}$, $\mathrm{NaOH}$ only at temperatures above $250^{\circ} \mathrm{C}$ [28]. While AlN films can be etched in hydroxyl based solutions [29], [30] and phosphoric acid at $80^{\circ} \mathrm{C} \mathrm{[31].} \mathrm{The} \mathrm{etch} \mathrm{rate} \mathrm{of} \mathrm{the}$ AlN films depends on the crystalline quality of the films. The etch rate of crystalline AlN films is much lower than polycrystalline films as shown in Figure 2.12. As shown in Figure 2.12 the etch rate of the AlN films decreases with increasing mean grain size of the films. The etching process starts at the defects. The higher etch rates in polycrystalline films is due to the existence of a higher defect concentration in polycrystalline films when compared single crystal films.

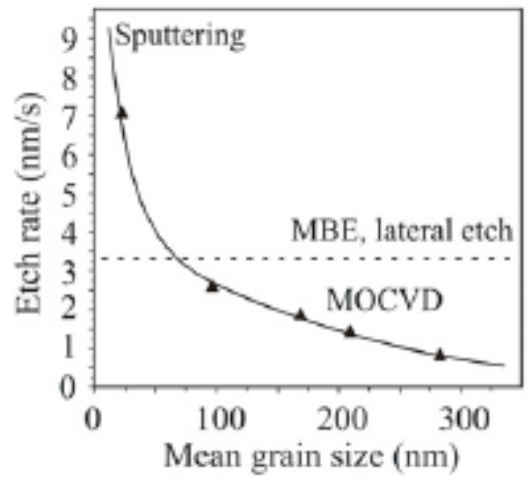

Figure 2.12: Etch rate of AlN films grown by different methods [30]. 
Further the etch rate of the AlN and GaN films is dependent on the polarity of the films. The N-phase is etched strongly than the metal phase [32].

\section{PEC etching [33], [34], [35]:}

In order to overcome the wet etching limitations due to the crystal quality and polarity of the sacrificial layer PEC techniques have been developed. PEC process is based on the enhancement of wet etching by ultraviolet (UV) radiation. The etching proceeds via oxidation of the semiconductor surface and subsequent dissolution of the surface oxides. The chemical equation explaining the etching process for $\mathrm{GaN}$ is shown below:

$$
\begin{aligned}
& 2 \mathrm{GaN}+6 h^{+}+6 \mathrm{OH}^{-} \rightarrow \mathrm{Ga}_{2} \mathrm{O}_{3}+3 \mathrm{H}_{2} \mathrm{O}+\mathrm{N}_{2} \uparrow \\
& \mathrm{Ga}_{2} \mathrm{O}_{3}+6 \mathrm{OH}^{-} \rightarrow 2 \mathrm{GaO}_{3}^{-3}+3 \mathrm{H}_{2} \mathrm{O}
\end{aligned}
$$

From the above chemical equation it can be seen that generation of holes $\left(\mathrm{h}^{+}\right)$is necessary for oxidation to occur. Irradiation of semiconductors by UV light with photon energy higher than the band gap leads to the excitation electrons to the conduction band. The excitation process also creates holes in the valence band. It is these holes which participate in the oxidation process. Further the oxidation process is enhanced through proper doping of the semiconductor. Therefore the photon energy and doping concentration are the two fundamental parameters which determine the etch rate and etch selectivity of the PEC process.

Bandgap selective etching can be performed by changing the emission wavelength of the UV source. Irradiation of a heterostructure, in which the bandgap of the functional and sacrificial layers are higher and lower than the photon energy respectively, results in the generation of holes in the sacrificial layer. The generation of holes in the sacrificial layer leads to a selective etching of the sacrificial layer. Figure 2.13 shows that lower band gap material have a larger lattice constant. Therefore the functional layer is usually tensile stressed which is beneficial for MEMS fabrication as it avoids buckling or out of plane deformation of the MEMS device. The process flow for the fabrication of a GaN cantilever by PEC is illustrated in Figure 2.13. 


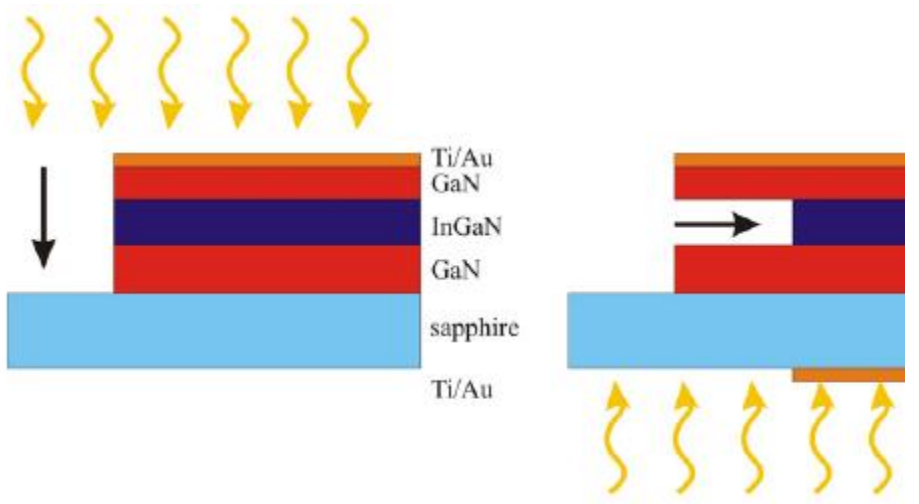

Figure 2.13: Process flow to fabricate GaN cantilever on sapphire substrate by PEC (a) masking from front side (b) masking from backside with GaN as light absorbing filter [1], [36].

This process is a fully PEC based etching technique. In the first step front side illumination is used to etch down to the sapphire by using the metal as a mask as shown in Figure 2.13 (a). The metal does not allow the light to propagate down to the underlying layers. Due to the high photon energy used, the light is absorbed by the all the layers under the unmasked area. The layers are unselectively etched down to the sapphire substrate surface. In the second step the etched structure is subjected to band gap selective etching through backside illumination to etch the InGaN layer. In addition the backside is patterned with metal to control the lateral etching. Further the thick GaN layer on the substrate absorbs photon energies close to the $\mathrm{GaN}$ bandgap from reaching the top GaN layer.

Difference in the doping of the functional and sacrificial layer can also be used to fabricate suspended structures using the PEC process. This process was used to realize an $\mathrm{n}-\mathrm{GaN}$ microfluidic channel on a p-GaN sacrificial layer [37]. The lateral etching was controlled by masking through Ni/Au electrodes as shown in Figure 2.14. 

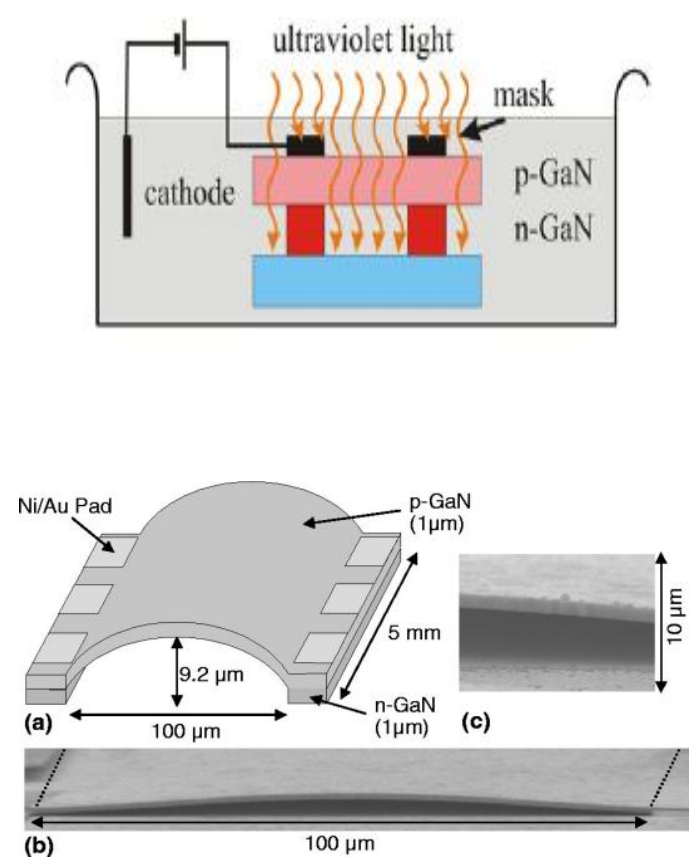

Figure 2.14: Schematic of doping selective PEC etch process for fabrication of GaN microfluidic channels [37]

This technology, which is an extension of photo-electrochemical etching, allows for the controlled and rapid undercutting of $p$-GaN epilayers. The control is achieved through the use of opaque metal masks to prevent etching in designated areas, while the high lateral etch rates are achieved by biasing the sample relative to the solution. For GaN microchannel structures processed in this way, undercutting rates in excess of $30 \mu \mathrm{m} / \mathrm{min}$ have been attained [37]. Based on the above techniques it can be concluded that IIInitride single crystal sacrificial layers can used for fabricating surface micromachined allnitride heteroepitaxial MEMS/NEMS devices on sapphire substrates. PEC techniques can be applied to selectively etch the underlying sacrificial layers both laterally and vertically. While PEC technique can be used to etch III-nitrides at reasonable at rates the technique does have some drawback:

Some of the drawbacks of the PEC techniques include:

- $\quad$ PEC is possible only when the energy of incident photon is higher than the energy of the band gap of the sacrificial layers. 
- The etching process may result in rough edges [38].

Nanocrysatlline thin films, oxides, metals, polymers are some of the commonly used sacrificial layers for fabricating MEMS devices. But MOVPE based III-nitride growth on these sacrificial layers has not been thoroughly investigated to fabricate MEMS/NEMS devices.

\section{Advantages of $\mathrm{SiO}_{\mathrm{x}}$}

Polymers, metals are not stable at high temperatures and are thus unsuitable for MOVPE growth. The nanocrystalline sacrificial films tend to recrystallize during the high temperature growth and thereby reduce the etch rates during the wet etching process. The recrystallization process also limits the growth temperature of the epitaxially overgrown layer [1]. Oxide based sacrificial layers such as silicon oxide $\left(\mathrm{SiO}_{\mathrm{x}}\right)$ has the potential to be used as sacrificial layers as they can sustain at high temperatures. Further the oxides can be deposited using several techniques such as PECVD, sputtering, e-beam evaporation etc and the wet etching techniques for silicon oxides sacrificial layers are well developed. Taking into consideration the aforementioned advantages of silicon oxides, this work demonstrates a process for fabricating MOVPE based thin film AIN airbridges using $\mathrm{SiO}_{\mathrm{x}}$ as a sacrificial layer.

\subsubsection{Lateral growth techniques III nitrides:}

Lateral growth of III-nitrides using $\mathrm{SiO}_{x}$ shown in Figure 2.7 allows for the reduction in the number of processing steps to etch the sacrificial layer. Lateral growth has been primarily developed for GaN. Shown in Figure 2.15 is a lateral growth process of GaN. 


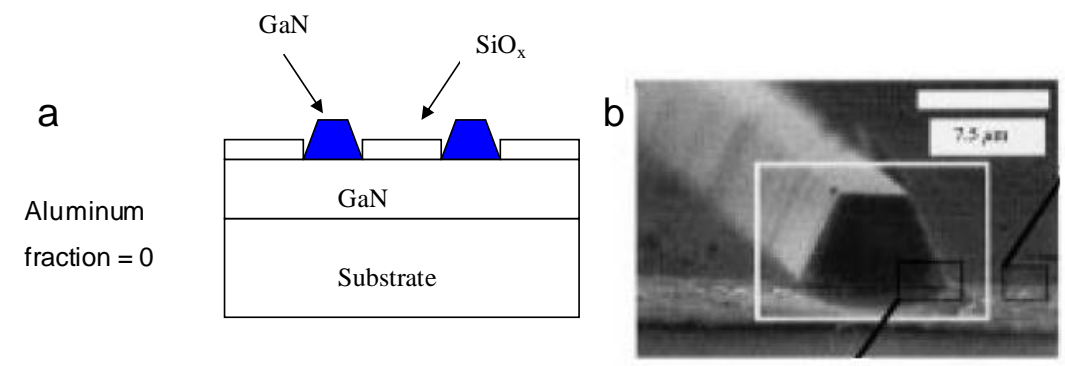

Figure 2.15: Lateral growth process of GaN (a) Schematic (b) SEM cross section image [39]

In this process the $\mathrm{GaN}$ surface is patterned with $\mathrm{SiO}_{\mathrm{x}}$ stripes. In the subsequent steps GaN growth is performed by MOCVD techniques. Due to the low sticking coefficient and high mobility of the Gallium adatoms on $\mathrm{SiO}_{x}$ GaN does not grow on $\mathrm{SiO}_{x}$ but preferably grows on $\mathrm{GaN}$ surface i.e in between the $\mathrm{SiO}_{\mathrm{x}}$ stripes as shown in Figure 9. However during the growth $\mathrm{Al}_{\mathrm{x}} \mathrm{Ga}_{1-\mathrm{x}} \mathrm{N}$ fims on $\mathrm{GaN}$ surface patterned with $\mathrm{SiO}_{\mathrm{x}}$ stripes ,AlGaN growth was observed on the stripes as well as shown in Figure 2.16. The growth on $\mathrm{SiO}_{\mathrm{x}}$ is due to the high sticking coefficient and low adatom mobility of aluminum on $\mathrm{SiO}_{\mathrm{x}}$. Moreover with increasing $\mathrm{Al}$ fraction in $\mathrm{Al}_{\mathrm{x}} \mathrm{Ga}_{1-\mathrm{x}} \mathrm{N}$ the thickness of of the film on $\mathrm{SiO}_{\mathrm{x}}$ was reported to be the same as that of the film on $\mathrm{GaN}$ surface between the stripes [39].

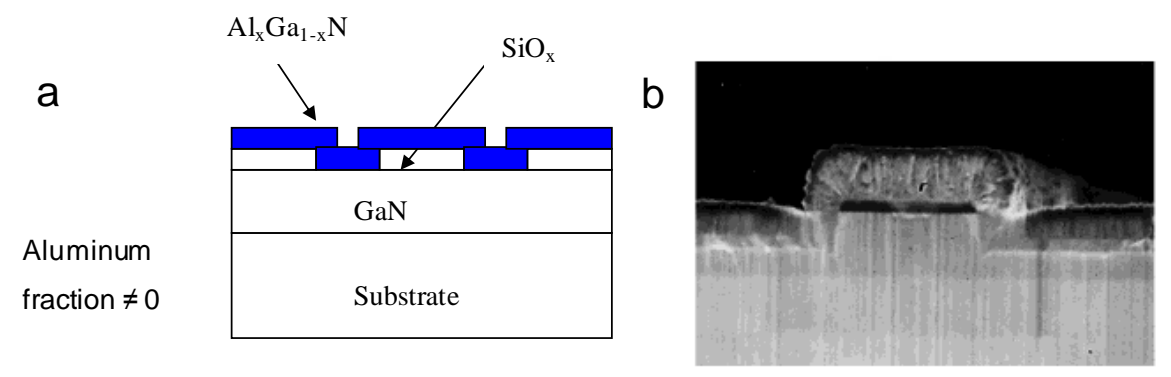

Figure 2.16: Lateral growth process of AlGaN (a) Schematic (b) SEM cross section image of $\mathrm{AlGaN}$ growth on $\mathrm{SiO}_{\mathrm{x}}$ with $40 \%$ aluminum [40] 
In the current work the lateral growth technique with AlN has been explored to fabricate suspended AlN air-bridges. 


\section{Chapter 3. Experimental Method}

\subsection{Flow process for fabricating AIN air-bridges:}

The flow process for fabricating AIN air-bridges is shown in Figure 3.1.

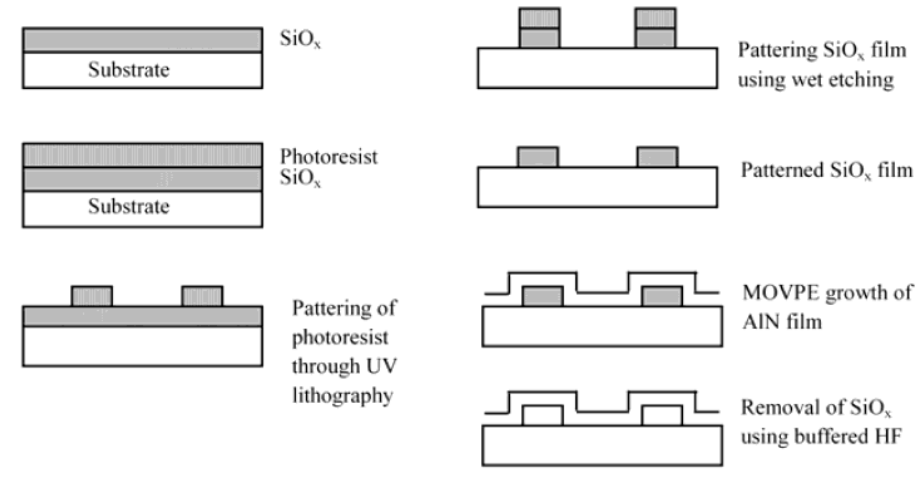

Figure 3.1: Flow process for the fabrication of thin film AlN air-bridge.

Initially $\mathrm{SiO}_{\mathrm{x}}$ films of thickness $130 \mathrm{~nm}$ were deposited on the substrates such as Si (111) and GaN/Sapphire templates using plasma enhanced chemical vapor deposition (PECVD) techniques. Due to the nature of the deposition technique the films were amorphous in nature. In the next step photo resist was used as a mask to etch the underlying $\mathrm{SiO}_{\mathrm{x}}$ sacrificial layer. The photo resist was patterned into stripes using standard UV lithography techniques. The width of the stripes ranged from $70 \mu \mathrm{m}$ to 100 $\mu \mathrm{m}$. The patterned samples were hard baked and subsequently etched in buffered HF $(10: 1)$ solution to remove the exposed $\mathrm{SiO}_{\mathrm{x}}$ layer. After the removal of the photo resist layer AlN films of thickness $300 \mathrm{~nm}$ were grown by MOVPE technique.

\subsection{Growth conditions for AIN films:}

AlN films were grown on the patterned substrates in an AIXTRON 200/4 RF-S MOVPE horizontal reactor. Trimethylaluminum and ammonia were used as the precursors with typical flows around $12 \mu \mathrm{mol} / \mathrm{min}$ and $1.5 \mathrm{slm}$ respectively. Hydrogen was used as the carrier gas with the total flow into the reactor being approximately 9000 
slm. All growths were conducted at a reactor pressure of 50 mbar and a temperature around $1100{ }^{\circ} \mathrm{C}$ resulting in an approximate growth rate of $4 \mathrm{~nm} / \mathrm{min}$. AlN thin films were grown on $\mathrm{Si}$ and $\mathrm{GaN} / \mathrm{Sapphire}$ substrates patterned with $\mathrm{SiO}_{\mathrm{x}}$ sacrificial layer. In order to fabricate suspended AlN structures it is necessary to remove the underlying sacrificial layer. The samples were etched in buffered HF (10:1) to remove the sacrificial layer to realize suspended AIN structures.

\subsection{Structural Characterization:}

Thermodynamically AlN is stable in a wurtzite phase. In order to fully exploit the piezoelectric properties of the AlN films for MEMS/NEMS device operation it is necessary that the AlN films exhibit a c-axis oriented wurtzite crystallography for optimum electromechnical coupling. The wurtzite structure consists of a hexagonal unit cell. In the wurtzite structure the $\mathrm{Al}$ and $\mathrm{N}$ atoms are tetrahedrally bonded to four $\mathrm{N}$ and four $\mathrm{Al}$ atoms respectively as shown in Figure 3.2. The wurtzite structures are characterized by lattice constants "a" and "c".

(a)
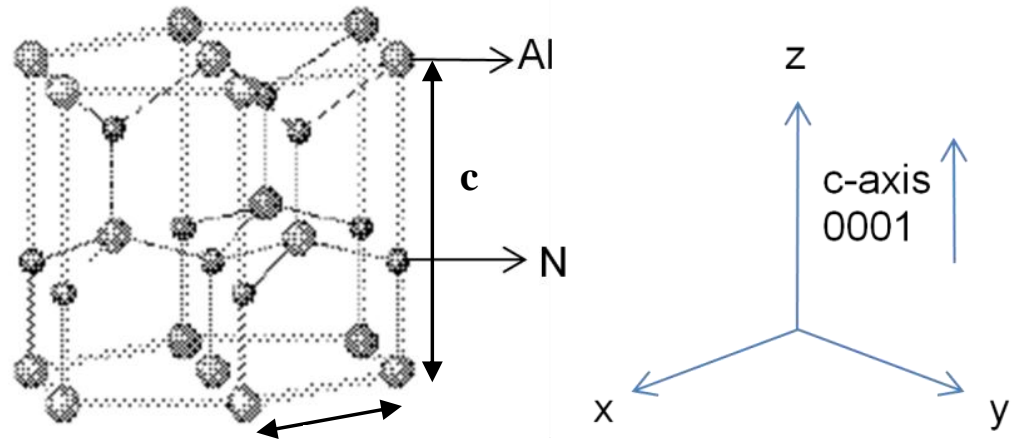

(b)

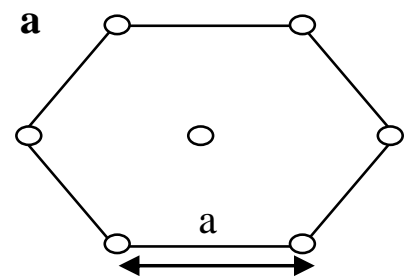

Figure 3.2: (a) Wurtzite crystallographic structure of AIN (b) Lattice points on the c plane [41]. 
It is known that in MOVPE growth techniques, the crystallographic orientation and crystal quality of the films primarily depend on the crystallographic structure of the surface on which the film is grown. Table 3.1 lists the properties of the AlN films grown by MOVPE techniques on $\mathrm{Si} \mathrm{(111)} \mathrm{and} \mathrm{GaN} \mathrm{(0001)} \mathrm{templates.}$

Table 3.1. Properties of AlN film grown on different substrates

\begin{tabular}{|l|l|l|}
\hline AIN & $\begin{array}{l}\text { "a - lattice } \\
\text { parameter lattice } \\
\text { mismatch }\end{array}$ & $\begin{array}{l}\text { Crystal } \\
\text { orientation }\end{array}$ \\
\hline $\mathrm{GaN}(0001)$ & $2.4 \%$ & c-axis \\
\hline $\mathrm{Si}(111)$ & $19 \%$ & c-axis \\
\hline $\mathrm{SiO}_{\mathrm{x}}$ & $?$ & $?$ \\
\hline
\end{tabular}

GaN films with c-axis oriented perpendicular to the thickness are preferred for the growth of c-axis oriented AlN films due to the similar wurtzite lattice structure. Further the lattice mismatch between AlN (0001) and GaN (0001) is only $2.4 \%$. The low lattice mismatch results in a heteroepitaxial high quality AlN film on GaN. For the growth of AlN(0001) films on $\mathrm{Si}$ substrates, which possesses a cubic crystallography, substrates oriented in (111) direction are preferred. Si (111) substrates offer a hexagonal symmetry which is similar in structure to c-axis oriented wurtzite crystals. However there exists a large lattice mismatch between AlN (0001) and Si (111). The mismatch results in misfit dislocations as shown in Figure 3.3. This results in films with inferior properties. 


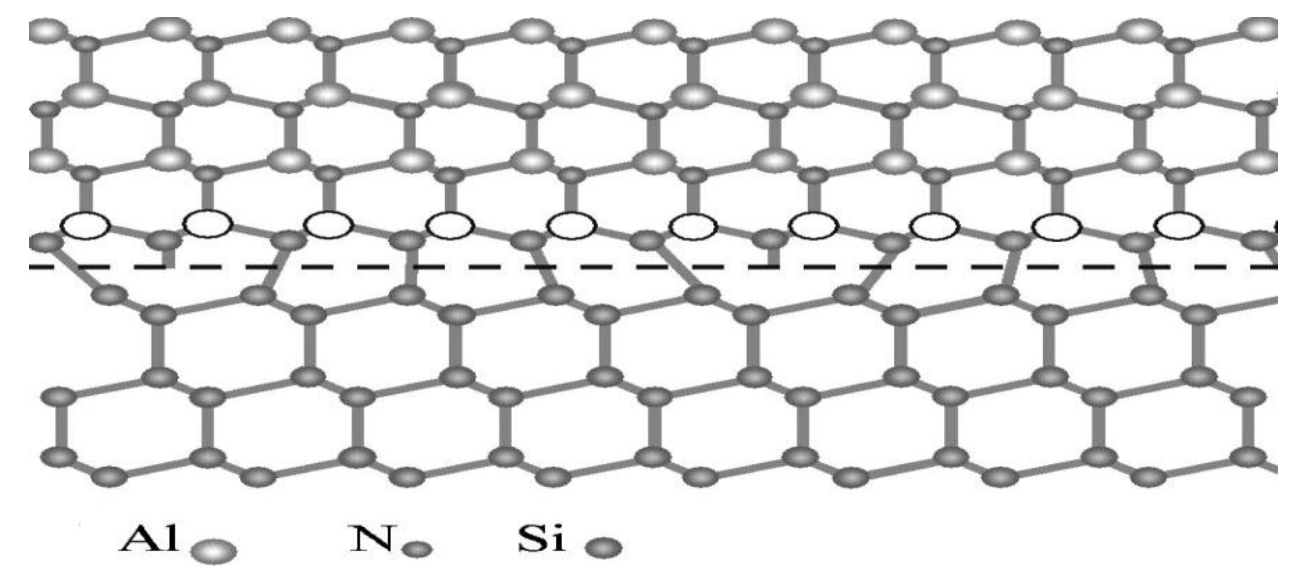

Figure 3.3: Atomic arrangement at the $\operatorname{AlN}(0001) / \mathrm{Si}(111)[42]$

Despite the large lattice mismatch, c-axis oriented AlN films with good structural properties and smooth surface morphology can be grown on $\mathrm{Si}$ (111) [43], [44]. In comparison to GaN and $\mathrm{Si}(111)$ the growth of AlN by MOVPE techniques on $\mathrm{SiO}_{\mathrm{x}}$ is expected to give films with inferior properties due to the amorphous nature of $\mathrm{SiO}_{\mathrm{x}}$. But the material properties of $\mathrm{AlN}$ films on $\mathrm{SiO}_{\mathrm{x}}$ such as the crystallographic orientation or piezoelectricity are unknown and have been rarely reported.

\subsubsection{X-ray diffraction (XRD):}

Typically XRD techniques have been used to study the crystal structure of thin films. This requires samples with lateral dimensions in the range of a few millimeters. But the lateral dimensions of a typical microfluidic/nanofluidic channels are much less than a millimeter. These dimensions do not allow the XRD techniques to unambiguously determine the crystal properties of the channels.

\subsubsection{Micro-Raman spectroscopy:}

A micro-Raman spectrometer combines both the aspects of a microscope and a Raman spectrometer. The setup allows the study of the material properties on the microscopic scale. 


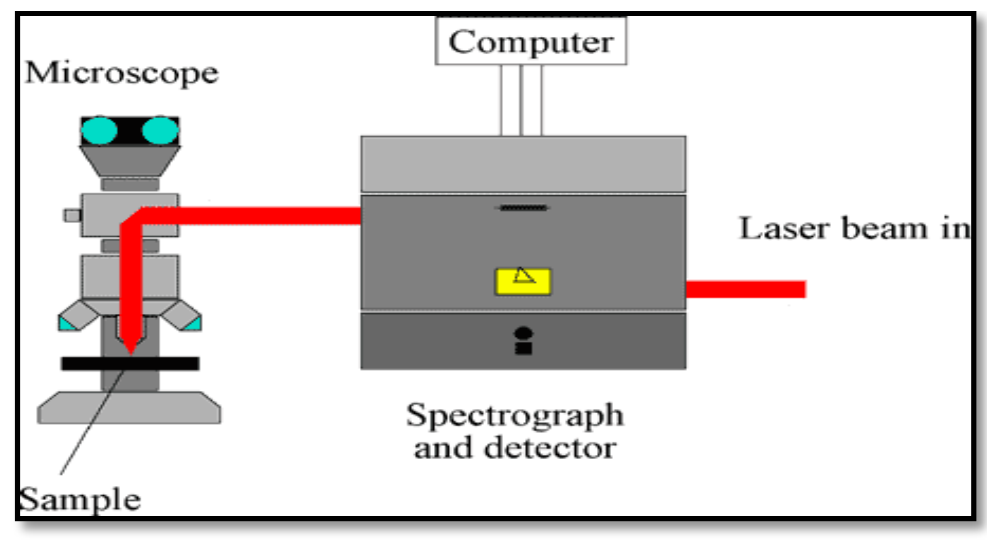

Figure 3.4: Micro-Raman spectrometer [45]

\subsubsection{Raman spectroscopy:}

The Raman spectroscopy is based on the optical phenomenon of inelastic scattering of light and was discovered by Sir C.V. Raman. When light is scattered from molecules most of the photons have same energy as that of the incident photons and this phenomenon is called the Rayleigh scattering. However, 1 in $10^{6}-10^{7}$ photons is scattered inelastically resulting in an exchange of energy between photon and molecules of the sample [46] as shown in Figure 3.5. The inelastic scattering of light is called the Raman scattering effect. Raman scattering is essentially an excitation of a molecule from a ground state to virtual state achieved through the process of photon-molecule interaction. 


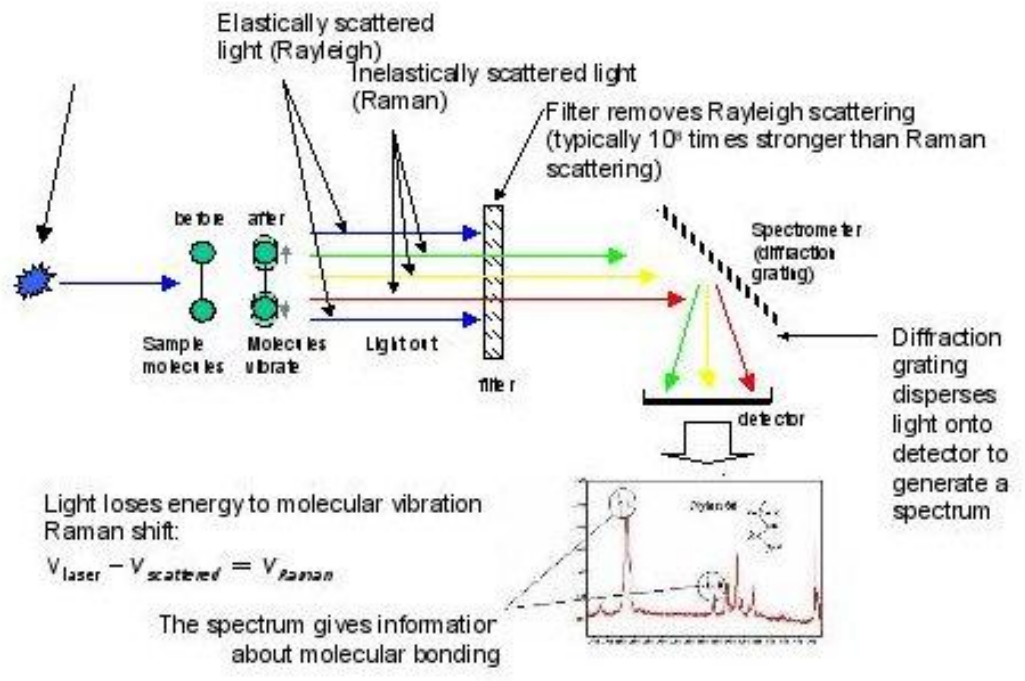

Figure 3.5: Raman scattering effect [47]

A change in the polarizability of the electron cloud in a molecule or the ability to induce an electric diople in the molecule is an essential condition for the molecule to exhibit the Raman scattering effect. The inelastic scattering of the incident photons leads to a change in the vibrational energy of the molecule. The energy levels of a molecule are quantized. The energy difference between the incident and scattered photon energies is therefore equal to the difference in the vibrational energies of the molecule as shown in Figure 3.6 through the Morse curves.

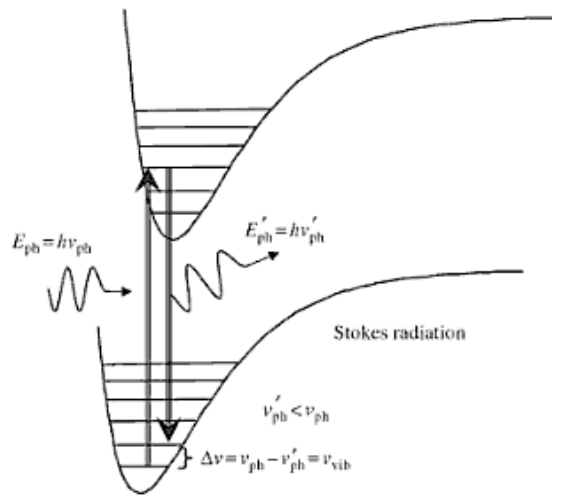

Figure 3.6: Illustration of Raman [48] 
Raman scattering is of two types namely Stokes and anti-Stokes as shown in Figure 3.7.

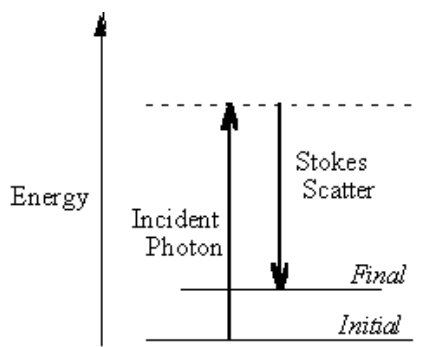

(a)

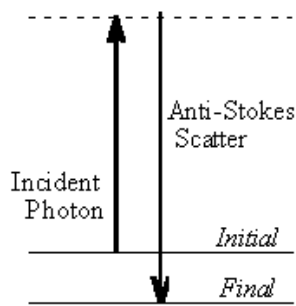

(b)

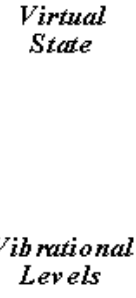

hationat

(1)

Figure 3.7: (a) Stokes shift (b) Anti-Stokes shift [49]

Stokes shift: In stokes shift, a molecule is excited by a photon the final vibrational state of the molecule has a higher energy than the initial vibrational state of the molecule, due to the exchange of energy between the molecule and the photon. The net result is that reemitted photons have lower energies than the incident photons. In the anti-Stokes shift the molecule absorbs photon and the emitted photon has higher energy.

The energy difference between the incident and scattered photons is usually expressed in terms of wavenumber. The Raman shift is given by:

$$
\bar{v}=\frac{1}{\lambda_{\text {incident }}}-\frac{1}{\lambda_{\text {scattered }}}
$$

The separation of the vibrational energy levels depends on the molecular structure. Some of the factors affecting the separation of the molecular vibration energy levels are the atomic mass, the bond order, molecular geometry etc. Typically a polyatomic molecule has more than one Raman vibrational mode resulting in multiple peaks in the Raman spectra. Each of the vibrational modes has a unique Morse curve.

\subsubsection{Raman selection rules [50]:}

Show in Figure 3.8 is a simplified molecular model in which the positive and negative centers of a molecule coincide as such the molecule is non polar. On applying 
an external electric field to the molecule the electric field induces dipoles in the molecule as shown in Figure 3.8. The molecule does not remain electrically neutral is essentially polarized in the presence of an external electric field.

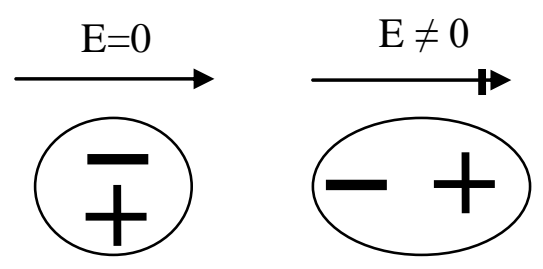

Figure 3.8: Dipole induced in a molecule due to external electric field

The polarizability of an electron cloud in a molecule is given by:

$$
P=\alpha E
$$

\section{$P$ : Induced dipole moment}

$\alpha$ : Polarizability

$E$ : Electric field

The time varying electric field is governed by the relation:

$$
E=E_{0} \cos \left(2 \pi v_{0} t\right)
$$

Where $v_{0}$ : Frequency of the incident electromagnetic wave.

Substituting Eq 3.3 into Eq 3.2 yields the time varying dipole moment:

$$
P=\alpha E_{0} \cos \left(2 \pi v_{0} t\right)
$$

The ability to perturb an electron cloud by an external electric field depends on the relative position of constitutive atoms in a molecule it follows that the polarizability also depends on the instantaneous position of the individual atoms. The displacement, $d Q$, of atoms in a time varying electric field can be expressed as:

$$
d Q=Q_{0} \cos \left(2 \pi v_{v i b} t\right)
$$

$v_{v i b}$ : is the frequency of vibration of the molecule frequency.

The displacement of the atoms is typically small compared to the bond length and for such small displacements the polarizability can be expressed using Taylor series as:

$$
\alpha=\alpha_{0}+\frac{\partial \alpha}{\partial Q} d Q
$$

where $\alpha_{0}$ is the polarizability of the molecule at equilibrium. By substituting Eq 3.5 into Eq 3.6 the polarizability can be further expressed as: 


$$
\alpha=\alpha_{0}+\frac{\partial \alpha}{\partial Q} Q_{0} \cos \left(2 \pi v_{v i b} t\right)
$$

Substituting the equation dipole moment equation yields

$$
P=\left(\alpha_{0}+\frac{\partial \alpha}{\partial Q} Q_{0} \cos \left(2 \pi v_{v i b} t\right)\right) E_{0} \cos \left(2 \pi v_{0} t\right)
$$

Using trigonometric identity Eq 3.8 can be further reduced to:

$$
P=\alpha_{0} E_{0} \cos \left(2 \pi v_{0} t\right)+\left(\frac{\partial \alpha}{\partial Q} \frac{Q_{0} E_{0}}{2}\right) \operatorname{eps}\left(2 \pi t\left(v_{v i b}-v_{0}\right)\right)+\cos \left(2 \pi t\left(v_{v i b}-v_{0}\right)\right)
$$

From Eq 3.9 it can be seen that an electromagnetic wave when incident on a molecule can induce oscillating dipoles which re-radiate light at three different frequencies at $\left(v_{v i b}-v_{0}\right),\left(v_{v i b}+v_{0}\right), v_{0}$. The first term is the Rayleigh scattering in which the scattered light has the same frequency as that of the incident light. The next two terms represent the Raman scattering, in which light is scattered at a frequency different from the incident light frequency. But the necessary condition for the inelastic

scattering to occur is that $\frac{\partial \alpha}{\partial Q} \neq 0$. In other words a change in polarizability of the molecule must occur along with a change in the displacement of the individual atoms. The term $v_{v i b}-v_{0}$ represents the stokes shift and the term $v_{v i b}+v_{0}$ represents the antistokes shift.

\subsubsection{Raman scattering under polarized incident light}

For a particular molecular vibration the polarization of the Raman scattered light is in the direction of the induced dipole moment. When the incident light has a polarization in the direction of the molecular vibration the scattered light will have the same polarization as the incident light. If the incident light induces a polarization in different direction the scattered light will have a polarization in the polarization induced direction. In the example show in Figure 3.9, the incident light has a polarization direction that is not aligned parallel to the direction of the induced dipole moment in the molecule [51]. 


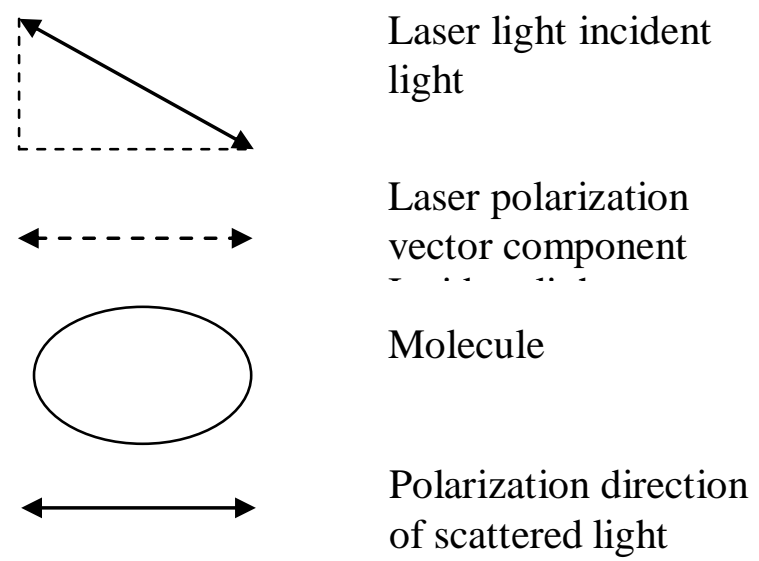

Figure 3.9: Polarization direction of Raman scattered with the direction of polarizability of the molecule not parallel to the source polarization direction [48]

However a component of the incident light electric field has a polarization direction parallel to the direction of polarizability of the molecule and it is this component that drives the Raman scattering process. The net result is that the scattered light has a polarization in the same direction as the polarizability of the molecule but different from the polarization direction of the incident light.

When polarized light interacts with a molecules, dipoles can be induced in each of the three Cartesian co-ordinates $\mathrm{x}, \mathrm{y}$, and $\mathrm{z}$. Thus the polarizability of a molecule is a tensor and can be expressed by following equation [52] :

$$
\left[\begin{array}{c}
P_{x} \\
P_{y} \\
P_{z}
\end{array}\right]=\left[\begin{array}{lll}
\alpha_{x x} & \alpha_{x y} & \alpha_{x z} \\
\alpha_{y x} & \alpha_{y y} & \alpha_{y z} \\
\alpha_{z x} & \alpha_{z y} & \alpha_{z z}
\end{array}\right]\left[\begin{array}{l}
E_{x} \\
E_{y} \\
E_{z}
\end{array}\right]
$$

where the first subscript in $\alpha$ represents the direction of polarizability of the molecule and the second subscript refers to the polarization of the incident light. If a molecule has a tendency to polarize in more than one direction then the scattered light will have polarization in the respective directions. 


\subsubsection{Lattice vibrations:}

Typically a solid sample under analysis consists of a large collection of molecules, ions or atoms. In a crystalline sample the atoms, molecules or ions are bonded together and shown in Figure 3.10 is the example of $\mathrm{NaCl}$ lattice structure. This periodic arrangement is called a crystal lattice.

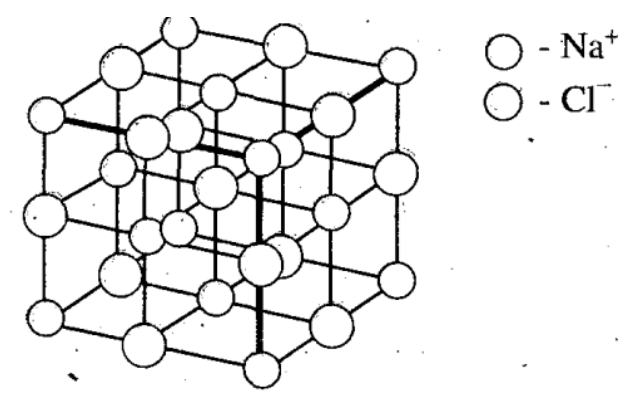

Figure 3.10: Lattice structure of $\mathrm{NaCl}[53]$

Molecules or atoms in a gas are free to move in random direction. But in a crystal these species are not free to move, as they are bound to each other through electrostatic forces. At absolute zero temperature or $0 \mathrm{~K}$ the species are not free to move. As the temperature of the sample rises above $0 \mathrm{~K}$ the species are set into thermal vibrations [54]. The energy is distributed throughout the lattice through mutual interaction between the species. These collective vibrations propagating as waves through the lattice are called lattice vibrations.

Typically a solid sample under analysis consists of a large collection of molecules, ions or atoms. In a crystalline sample the atoms, molecules or ions are bonded together and arranged in an orderly repeating pattern in a three dimensional space. This periodic arrangement is called a crystal lattice. The concept of lattice vibrations can be understood by considering a simple one dimensional linear chain consisting of two atoms with mass $\mathrm{m}$ and $\mathrm{M}$, connected by springs. The diatomic atoms are placed alternately and separated by a distance $a$ as shown in Figure 3.11. 


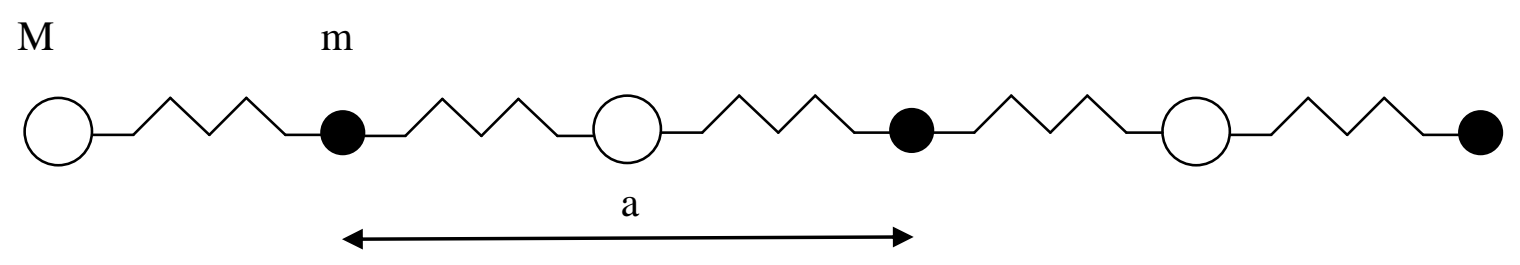

Figure 3.11: A one dimensional linear chain with a diatomic lattice [55].

In this arrangement of atoms the displacement of an atom from its equilibrium created in an atom will lead to a disturbance in the neighboring atoms. Assuming that the atom is influenced by its nearest neighbor and that the displacement from equilibrium position obeys Hookes law. The equation for the motion of nth atom is given by [55]:

$$
\begin{gathered}
M \frac{d^{2} u_{n}}{d t^{2}}=-q\left(u_{n}-v_{n}\right)-q\left(u_{n}-v_{n-1}\right)=-q\left(2 u_{n}-v_{n}-v_{n-1}\right) \\
m \frac{d^{2} v_{n}}{d t^{2}}=-q\left(v_{n}-n_{n+1}\right)-q\left(v_{n}-u_{n}\right)=-q\left(2 v_{n}-u_{n+1}-u_{n}\right)
\end{gathered}
$$

where $u_{n}, v_{n}$ are the displacements of the nth atoms $\mathrm{M}$ and $\mathrm{m}$ respectively.

As the waves are traveling waves the displacements can be expressed as

$$
\begin{gathered}
u_{n}=A \exp j\left(\omega t-k x_{n}\right) \\
v_{n}=B \exp j\left(\omega t-k\left(x_{n}+\frac{1}{2} a\right)\right)
\end{gathered}
$$

substituting Eq 3.11 in Eq 3.10 gives:

$$
\begin{aligned}
& -\left(M \omega^{2}+2 q\right) u_{n}=\left(-m \omega^{2}+2 q\right) v_{n} \\
& q\left(1+e^{-j k a}\right) u_{n}=\left(-m \omega^{2} 2 q\right) v_{n}
\end{aligned}
$$

Solution for these simultaneous equations gives :

$$
\omega^{2}=q\left(\left(\frac{1}{M}+\frac{1}{m}\right) \pm q \sqrt{\left(\frac{1}{M}+\frac{1}{m}\right)^{2}-\frac{4 \sin ^{2}(k a / 2)}{M m}}\right.
$$


where $\omega$ is the angular frequency of the traveling wave and $k$ is the wave vector. relation between $\omega$ and $k$ and is called the dispersion relation. Of the possible solutions for the above equation the positive solutions for $\omega$ give the traveling wave equations. For small $k$ we can approximate

$$
\begin{gathered}
\sin \left(\frac{k a}{2}\right)=\frac{k a}{2} \\
\omega_{+}=\omega_{0}\left[1-\frac{m M(k a)^{2}}{8(M+m)^{2}}\right] \\
\omega_{0}=\sqrt{2 q\left(\frac{1}{M}+\frac{1}{m)}\right.} \\
\omega_{-}=k a \sqrt{\frac{q}{(M+m)}}
\end{gathered}
$$

A plot of $\omega$ vs $k$ within the first Brillouin zone i.e from $k=\frac{\pi}{a} t o-\frac{\pi}{a}$,for a linear chain of atoms, is shown in Figure 3.12. Each k represents a lattice vibration mode.

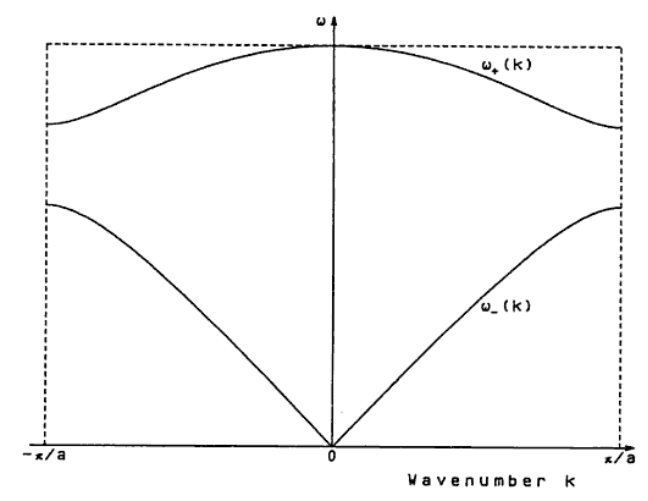

Figure 3.12: Dispersion relation of $\omega_{-}$and $\omega_{+}$with respect to wave number $k$ in the first Brillouin zone of a linear chain of atoms [55].

The top branch is called the optical branch and the lower branch is the optical mode. At $k$ $\approx 0$

$$
\begin{aligned}
& \frac{v_{n}}{u_{n}} \cong-\frac{M}{m}-\text { Optical branch } \\
& \frac{v_{n}}{u_{n}} \cong 1 \quad \text { - Acoustic branch }
\end{aligned}
$$


In the optical mode the atoms displace in opposite direction. If the two atoms are oppositely charged the lattice vibrations can be activated by infrared optical radiation. Therefore the lattice vibrational mode is called optical vibrational mode. The vibrations are of two types transverse optical (TO) and longitudinal optical (LO) as shown in Figure 3.13

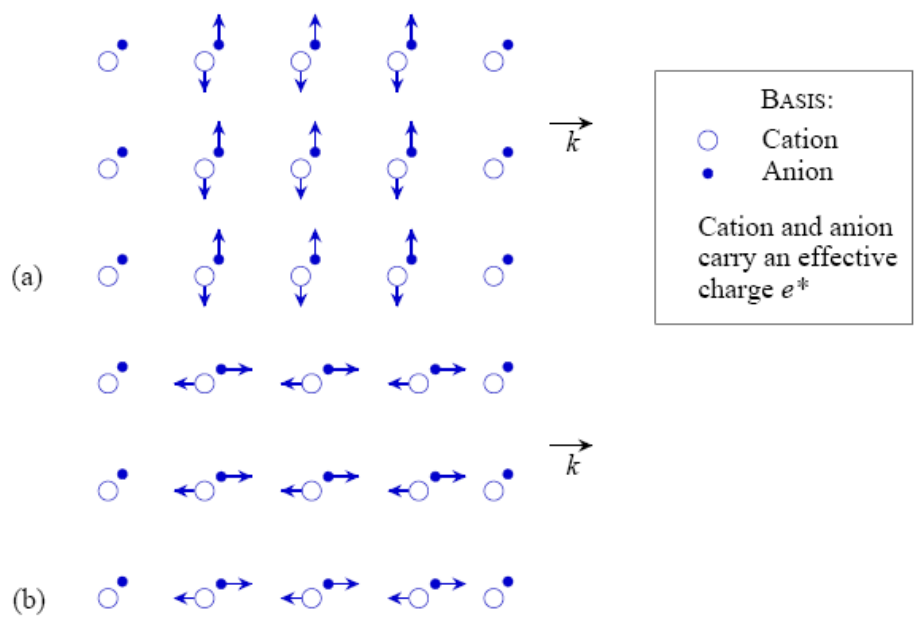

Figure 3.13: (a) Transverse optical (b) Longitudinal optical lattice vibrations modes in a diatomic unit cell [56]

\subsubsection{Phonons:}

According to the classical theory, a lattice vibrations exists for every $k$, however the quantum theory predicts that the energy of the lattice vibrations is quantized. These quantized lattice vibrations are called phonons. The allowed energies for lattice vibrations are given by the following equation [56]:

$$
E_{n}=h v\left(n+\frac{1}{2}\right), \text { where }(\mathrm{n}=0,1,2,3 \ldots)
$$

In order to observe the phonons the necessary condition for inelastic process to occur is the conservation of energy and momentum [57].

$$
\begin{gathered}
\vec{k}_{i}=\vec{k}_{s}+k \\
\hbar \omega_{i}=\hbar \omega_{s}+\hbar \Omega
\end{gathered}
$$


Compared to the energy of the visible or infrared radiation the energy of a phonon is less small this leads to $\omega_{i}$ and $\omega_{s}$ being almost equal. The lattice parameter of a crystal is typically a few angstroms and the wave vector of these electromagnetic waves is comparable to the wave vector of the phonons only at the zone center i.e at $k=0$. Thus phonons at the zone center only can be investigated by optical techniques such as Raman scattering.

\subsubsection{Zone center phonons of wurtzite Aluminum Nitride (AIN)}

Wurtzite AIN has four atoms per unit cell and is a uniaxial crystal as shown in Figure 3.14(a). For a wurtzite structure, group theory predicts the existence eight phonon modes at the zone center. The eight phonon modes are, viz. $2 \mathrm{~A}_{1}+2 \mathrm{E}_{1}+2 \mathrm{~B}_{1}+2 \mathrm{E}_{2}$ as shown in Figure 3.14(b) [41].

(a)

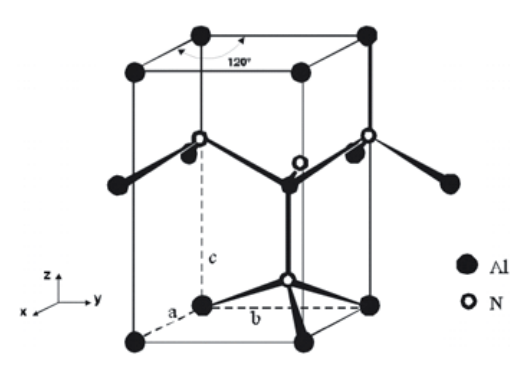

(b)

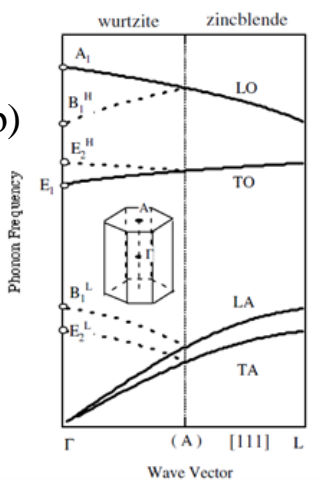

Figure 3.14: (a) Unit cell of AlN crystal [58] (b) Zone center optical phonons in a wurtzite crystal [41]

Among these zone center phonon modes one set of $A_{1}$ and $E_{1}$ modes are acoustic modes while the remaining six i.e $\mathrm{A}_{1}+\mathrm{E}_{1}+2 \mathrm{~B}_{1}+2 \mathrm{E}_{2}$ are the optical phonon modes. The atomic displacements of the optical modes are shown in Figure 3.15. The $A_{1}$ and $B_{1}$ modes represent atomic displacements parallel to the c-axis, while the $E_{1}$ and $E_{2}$ modes represent atomic displacements perpendicular to the c-axis. The $A_{1}$ and $E_{1}$ modes are both Raman and infrared active modes, while both the $\mathrm{E}_{2}$ modes are only Raman active and the $B_{1}$ mode is neither Raman active nor infrared active [59]. Further $A_{1}$ and $E_{1}$ split into TO and LO modes due to long range electrostatic forces. 

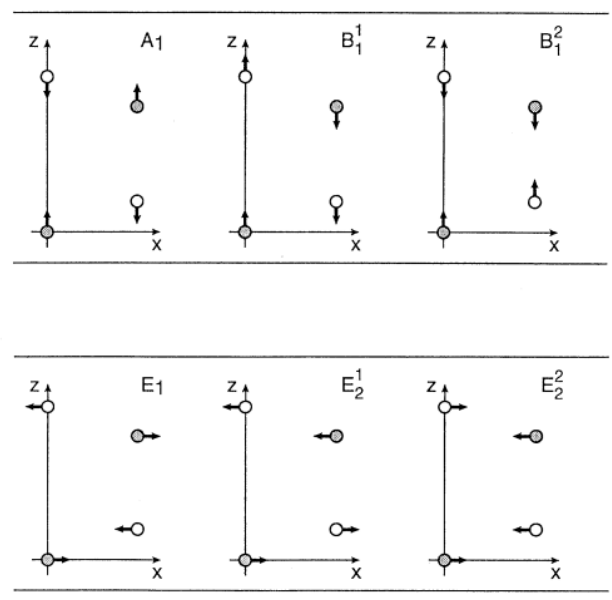

Figure 3.15: Optical phonons in a wurtzite crystal [60].

For a unit cell having $\mathrm{N}$ atoms the number of optical modes are $3 \mathrm{~N}-3$ [61]. A wurtzite structure has four atoms per unit cell which is expected to give rise to nine optical phonon modes. The optical phonon modes are A1 (LO), two $\mathrm{E}_{1}$ (TO), two $\mathrm{E}_{2}$ (low), two $\mathrm{E}_{2}$ (high), one $\mathrm{B}_{1}$ (low) and one $\mathrm{B}_{1}$ (high). Due to the degeneracy of the $\mathrm{E}_{1}$ (TO), $E_{2}$ (low), and $E_{2}$ (high) pairs only six optical phonon modes exist. Of the two acoustic phonon modes two E1 (TA) modes are degenerate.

The Raman selection rules for observing the optical phonons modes are listed in Table 3.2 as shown below :

Table 3.2: Raman configuration of allowed modes in wurtzite crystals [41]

\begin{tabular}{ll}
\hline Configuration & Mode \\
\hline$x(y, y) \bar{x}$ & $\mathrm{~A}_{1}(\mathrm{TO}), \mathrm{E}_{2}$ \\
$x(z, z) \bar{x}$ & $\mathrm{~A}_{1}(\mathrm{TO})$ \\
$x(z, y) \bar{x}$ & $\mathrm{E}_{1}(\mathrm{TO})$ \\
$x(y, z) y$ & $\mathrm{E}_{1}(\mathrm{TO}), \mathrm{E}_{1}(\mathrm{LO})$ \\
$x(y, y) z$ & $\mathrm{E}_{2}$ \\
$z(y, x) \bar{z}$ & $\mathrm{E}_{2}$ \\
$z(y, y) \bar{z}$ & $\mathrm{~A}_{1}(\mathrm{LO}), \mathrm{E}_{2}$ \\
\hline
\end{tabular}

The above notation is the porto notation. For example when considering the backscattering geometry $z(y, x) \bar{z}$ implies that the incident laser is travelling along $\mathrm{z}$ direction with polarization in $y$ direction and the detected light is travelling back in the 
negative $\mathrm{z}$ direction and is being inspected for electric fields polarized in the $y$ direction. Typically the c-axis of the wurtzite crystal is taken as the positive $\mathrm{z}$ direction. $x$ and $y$ are any two perpendicular axis in the a-plane and normal to the c-axis. 


\section{Chapter 4. Properties of AIN films on $\mathrm{SiO}_{\mathrm{x}}$}

\subsection{Determination of crystal orientation by polarized Micro-Raman spectroscopy}

$\mathrm{SiO}_{\mathrm{x}}$ films of thickness $130 \mathrm{~nm}$ were deposited on substrates using Plasma Enhanced Chemical Vapor Deposition (PECVD). The films were patterned into stripes, with widths of $80 \mu \mathrm{m}$ to $110 \mu \mathrm{m}$, using standard photolithography and wet etching techniques. AlN films $300 \mathrm{~nm}$ thick were subsequently grown on the patterned substrates by MOVPE. The flow process for the fabrication of an AIN thin film air-bridge is shown in Figure 4.1.
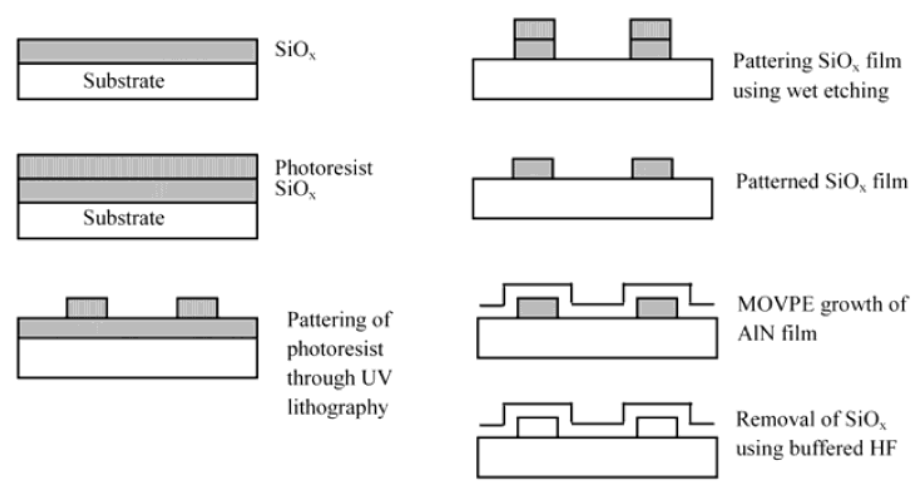

Figure 4.1: Flow process for the fabrication of an AlN thin film air-bridge.

The growth conditions for the AlN films have been reported elsewhere [62]. In this study Renishaw in via Raman microscope was used to conduct micro-Raman analysis at room temperature. The laser excitation wavelength was $514 \mathrm{~nm}$ and was focused to a spot having a diameter around $3 \mu \mathrm{m}$. To identify the Raman phonon modes corresponding to wurtzite crystal structure the measurements were taken in the $z(x, y) \bar{z}$ backscattering geometry [41].

For wurtzite crystals exhibiting a c-axis orientation the Raman modes that are observed in the backscattering geometry $z(x, y) \bar{z}$ are the $\mathrm{E}_{2}$ (high) and $\mathrm{E}_{2}$ (low) modes respectively. The Raman spectra of $\mathrm{AlN} / \mathrm{SiO}_{\mathrm{x}}$ bilayer on $\mathrm{GaN}$ and $\mathrm{Si}$ (111) templates are shown in Figure 4.2. 

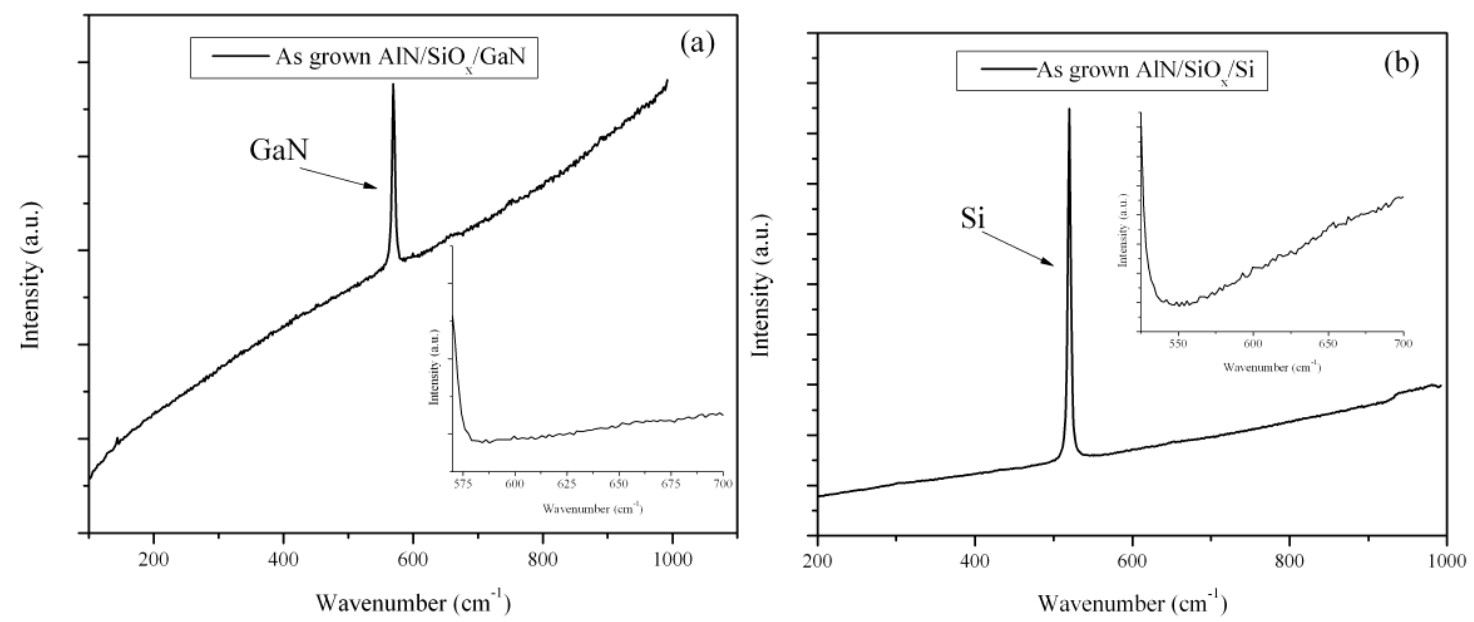

Figure 4.2: Raman spectra of as-grown AlN film on $\mathrm{SiO}_{\mathrm{x}}$ (a) $\mathrm{GaN} / \mathrm{Sapphire}$

(b) Si (111).

The dominant peaks superimposed on the baseline in Figure 4.2 (a) and (b) correspond to the Raman peaks from the $\mathrm{GaN}$ and $\mathrm{Si}$ templates respectively. As the band gap of $\mathrm{Si}$ is lower than the incident photon energy of $2.4 \mathrm{eV}$ the Raman scattering intensity from $\mathrm{Si}$ is higher than that of $\mathrm{GaN}$ as shown in Figure 4.2. It can also be seen from the inset spectra in Figure 4.2 (a) and Figure 4.2 (b) that the Raman modes of the AlN film, which were expected to be superimposed on the fluorescence background between $600 \mathrm{~cm}^{-1}$ to $700 \mathrm{~cm}^{-1}$ [63] cannot be clearly discerned. Therefore the Raman peaks corresponding to AlN on $\mathrm{SiO}_{x}$ could not be identified immediately after growth. But under the same measurement conditions the Raman modes corresponding to c-axis oriented AlN films grown directly on $\mathrm{Si}$ (111) and GaN surfaces were clearly observed. The apparent absence of the AIN Raman modes is most likely due to the lower crystal quality of the AlN film. Scanning electron microscope (SEM) images shown in Figure 4.3 indicate that the AlN films on $\mathrm{SiO}_{\mathrm{x}}$ contain randomly oriented crystals typical of a polycrystalline film. The degradation in the crystal quality is expected and attributed to the amorphous nature of the sacrificial layer. 


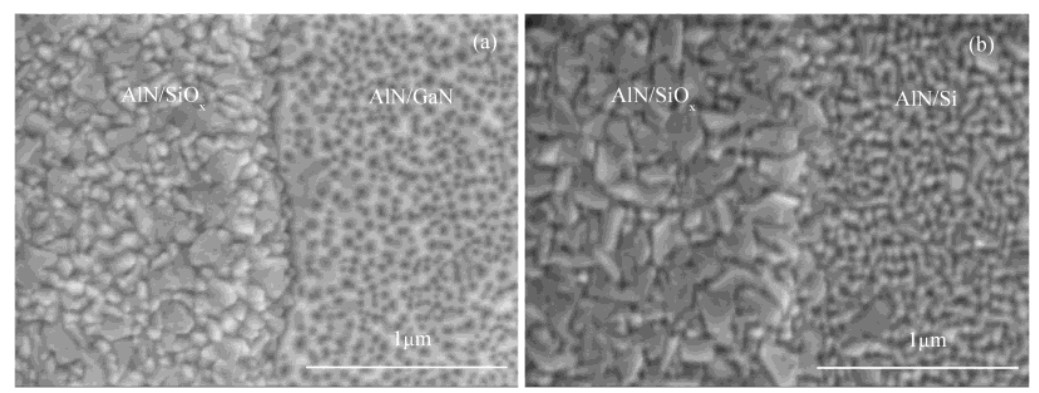

Figure 4.3: SEM images of as-grown AlN films on (a) GaN (b) Si (111) templates patterned with $\mathrm{SiO}_{\mathrm{x}}$.

\subsection{Etch rate of AIN in $\mathrm{AZ} \mathrm{400K}$ solution and crystal quality:}

Literature has reported that the etch rate of AIN in AZ400K is dependent on the material properties of the AlN films. Polycrystalline films are etched more rapidly than crystalline films [64]. In order to qualitatively asses the crystal quality of the AlN films grown on $\mathrm{SiO}_{\mathrm{x}}$ the as-grown samples were etched in undiluted $\mathrm{AZ400K}$. AZ400K is a known photoresist developer. Figure 4.4 shows the SEM cross section images of the samples after etching in AZ400K solution for 20 min.
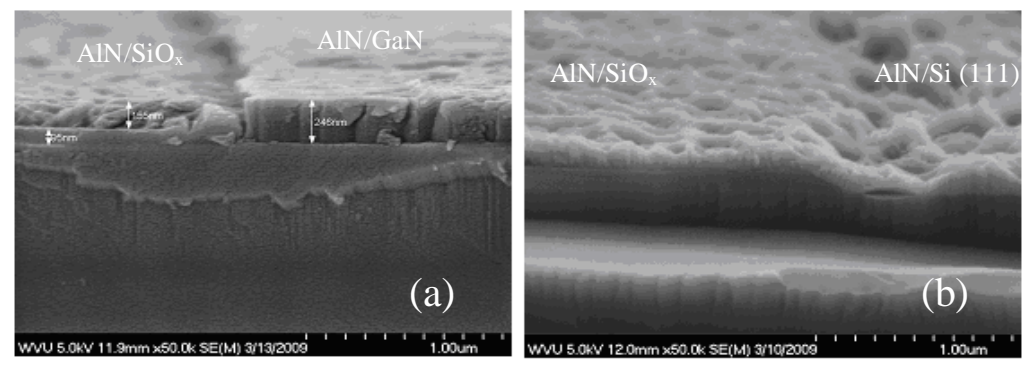

Figure 4.4: SEM cross-section of AlN films on (a) GaN (b) Si (111) after wet etching in AZ 400K for 20minutes

Thickness of the AlN films grown on these samples was equal to $300 \mathrm{~nm}$ on both the $\mathrm{SiO}_{\mathrm{x}}$ and the substrate. From Figure 4.4 (a) it can be seen that, after a 20 min etching process in AZ400K, the thickness of AlN on GaN has been reduced by only $60 \mathrm{~nm}$ while the AlN films on $\mathrm{SiO}_{\mathrm{x}}$ has been significantly reduced. Observation of SEM cross-section images of AlN films grown on $\mathrm{Si}$ (111) substrates shows that the AlN films on $\mathrm{SiO}_{\mathrm{x}}$ and $\mathrm{Si}$ (111) have been significantly etched. It is known that the "a lattice parameter' lattice 
mismatch between AlN and GaN is only $2.4 \%$. Therefore the growth of AlN on GaN is expected to yield high quality crystalline films with fewer defects. But the in plane lattice mismatch between AlN on Si (111) is around $19 \%$ and therefore the growth of AlN on Si (111) results in films with more defects. Due to the above reasons the AlN films on GaN have a lower etch rate than the AlN films on Si (111). Based on the etch rate crystal quality relation it can be concluded that due the AlN films on $\mathrm{SiO}_{\mathrm{x}}$ have a lower crystal quality than the AlN films grown on GaN. Figure 4.5 shows the SEM surface images of the AlN films on $\mathrm{SiO}_{\mathrm{x}}$ and $\mathrm{Si}$ (111) after a 20 min etching. It can be seen that the AlN films on Si (111) have geometrical structures and these regular structures on the surface can be attributed to the influence of the underlying crystalline $\mathrm{Si}$ (111) substrate.

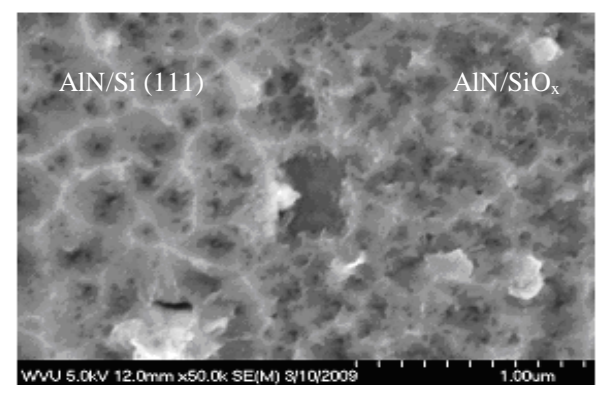

Figure 4.5: SEM surface image of $\mathrm{AlN}$ on $\mathrm{SiO}_{\mathrm{x}}$ and $\mathrm{Si}$ (111) after wet etching in AZ400K

But the AlN film on $\mathrm{SiO}_{\mathrm{x}}$ does not appear to have any geometrical shapes and it follows that the AlN films take on the crystal structure of the underlying $\mathrm{SiO}_{\mathrm{x}}$ and therefore have a lower crystal properties when compared to AlN films grown on $\mathrm{Si}$ (111) and GaN templates.

The wet etching-crystal quality relation provides further insight into the reasons for the degradation in the quality of the AlN films on amorphous sacrificial layers. The degradation of the AlN films was shown to produce weak Raman spectra [65]. Further due to the background noises originating from strong fluoresce of the underlying amorphous layer the observation of Raman spectra is made even more difficult, as the relatively weak Raman modes were lost in the background noise. The fluorescence from 
the underlying amorphous sacrificial layer can be avoided by using long wavelength light. Due to the non availability of longer wavelengths on the current Raman setup alternate techniques had to be investigated.

\subsection{Identifying crystallographic orientation by Micro-FTIR techniques:}

The material properties of AlN thin films such as the crystallographic orientation of have been extensively studied by Raman spectroscopic techniques [66], [67], [68] [69]. But the study of crystallographic orientation of AlN thin films by infrared transmission techniques has been investigated to a lesser extent [70], [71]. Further Micro-FTIR techniques have been rarely applied to investigate the properties of AlN thin film MEMS/NEMS structures.

\section{Berreman effect:}

For a polar crystal the frequency dependent refractive index is given by:

$$
n^{2}(\omega)=\epsilon_{\infty}+\epsilon_{\infty} \frac{\omega_{\mathrm{LO}}^{2}-\omega_{\mathrm{TO}}^{2}}{\omega_{\mathrm{TO}}^{2}-\omega^{2}-i \Gamma \omega}
$$

Where and are the transverse optical and longitudinal optical infrared phonon modes. For the refractive index of the material are negative. Therefore at these frequencies no light can propagate through the film as shown in Figure 4.6.

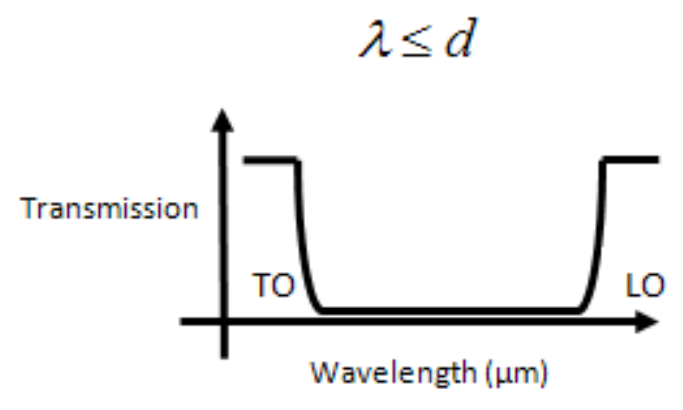

Figure 4.6: Infrared transmission spectra of a polar optical crystal in the Restrahelen band for thick films. 
D.W. Berreman in 1963 demonstrated that for polar films whose thickness is much less than the wavelength of the incident infrared light and at an oblique angle of incidence the transmission is significantly improved. Under the above conditions the natural resonance modes i.e the TO and LO modes can be clearly observed in the transmission mode as shown in Figure 4.7.

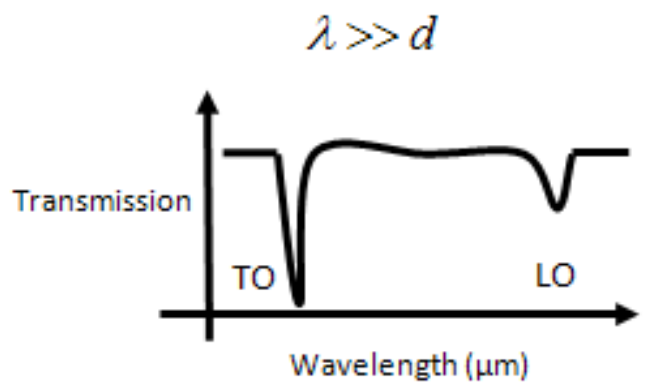

Figure 4.7 : Infrared transmission spectra of a polar optical crystal in the Restrahelen band for thin films.

This condition is easily satisfied for films having thickness of a few hundred nanometers.

However due to the uniaxial crystallography of the wurtzite structure the optical response depends on the crystallographic orientation of the films. Figure 3.25 shows the relative displacements of the $\mathrm{Al}$ and $\mathrm{N}$ atoms in the unit cell of a wurtzite AlN structure. In the unit cell the $\mathrm{Al}$ atom has a relatively positive charge with respect to the $\mathrm{N}$ atom due to the difference in the electronegativity between the $\mathrm{Al}$ and the $\mathrm{N}$ atoms. Figure 3.25 shows the $\mathrm{E} 1$ mode in which the $\mathrm{Al}$ and the $\mathrm{N}$ atom tend to displace in the direction opposite to each other and also perpendicular to the c-axis. This results in a change in the net dipole moment in the unit cell, which is the necessary condition for a molecule to be infrared active. Therefore the E1 mode is infrared active. Based on similar analysis the other infrared active mode is the A1 mode. Table 4.1 shows the observable infrared active phonon modes in a wurtzite crystal according to the Berreman effect 
Table 4.1: Infrared activity of wurtzite crystals

\begin{tabular}{lcccccc}
\hline \hline & \multicolumn{2}{c}{$\theta_{i}=0$} & \multicolumn{2}{c}{$\theta_{i} \neq 0, s$ polarization } & \multicolumn{2}{c}{$\theta_{i} \neq 0, p$ polarization } \\
& $\mathbf{E} \| \hat{c}$ & $\mathbf{E} \perp \hat{c}$ & $\hat{c} \| \mathbf{I}$ & $\hat{c} \perp \mathbf{I}$ & $\hat{c} \| \mathbf{I}$ & $\hat{c} \perp \mathbf{I}$ \\
\hline$\hat{c} \| f$ & $A_{1}(\mathrm{TO})$ & $\mathrm{E}_{1}(\mathrm{TO})$ & $E_{1}(\mathrm{TO})$ & $A_{1}(\mathrm{TO})$ & $A_{1}(\mathrm{TO}), E_{1}(\mathrm{LO})$ & $E_{1}(\mathrm{TO}), E_{1}(\mathrm{LO})$ \\
$\hat{c} \perp f$ & & $E_{1}(\mathrm{TO})$ & $E_{1}(\mathrm{TO})$ & & $E_{1}(\mathrm{TO}), A_{1}(\mathrm{LO})$ & \\
\hline \hline
\end{tabular}

[73]

For investigating structures with lateral dimension as low as $100 \mu \mathrm{m}$ infrared microsampling can be used as shown in Figure. In the micro-infrared technique, rays from an unpolarized infrared source converge on the sample at an oblique angle of incidence. Therefore for a c-axis oriented film, infrared transmission measurements under an oblique angle of incidence must show the E1 (TO) and A1 (LO) modes only.
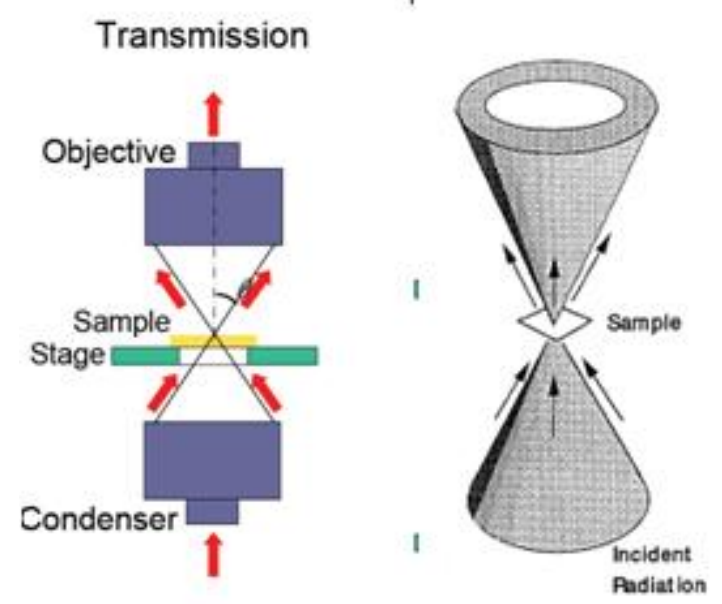

Figure 4.8: (a) Micro-FTIR transmission measurement setup [Varian Inc] (b) Oblique angle of incidence on the infrared rays in a micro-FTIR [74]

\section{Experimental details}

$\mathrm{SiO}_{\mathrm{x}}$ films of thickness $130 \mathrm{~nm}$ were deposited on $\mathrm{Si}$ (111) substrates using plasma enhanced chemical vapor deposition (PECVD). The films were patterned into stripes, with widths of $110 \mu \mathrm{m}$, using a standard photolithography and wet etching process. Using MOVPE growth techniques, AIN films $300 \mathrm{~nm}$ thick were grown on the patterned substrates. The process flow for the fabrication of a nanofluidic channel is shown in Figure 4.9. The growth conditions for the AlN films are presented elsewhere 
[62]. The samples were post processed by etching in buffered HF (10:1) solution for different etching times.

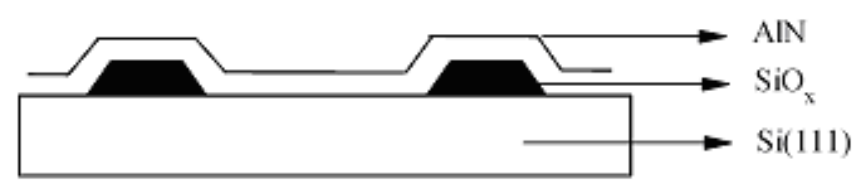

Wet etching

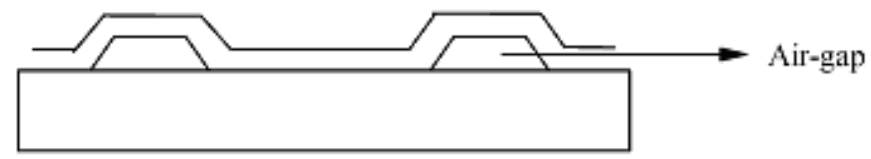

Figure 4.9: Process flow for the fabrication of an AlN thin film suspended structure.

Infrared transmission spectra were taken at room temperature using a Digilab UMA 600 microscope under unpolarized conditions from $400-4000 \mathrm{~cm}^{-1}$ with a spectral resolution of about $2 \mathrm{~cm}^{-1}$. The dimension of the aperture was fixed at $100 \mu \mathrm{m} \times 1000 \mu \mathrm{m}$ for all conditions. Absorption bands from bare Si (111) substrate were subtracted from the AlN/Si (111) spectra. The transmission spectrum is the response of the sample over a range of incident angles within the cone of illumination with a solid angle of $30^{\circ}$. Lorentz functions were fitted to the AIN absorption bands to determine the peak position and integrated area. For performing nonlinear peak separation the software analysis package PeakFit was utilized. During the fitting process the PeakFit program was allowed to automatically detect the peaks and subsequently least square minimization was performed to obtain the best fit. FTIR measurements were taken at several areas and the weighted average of the integrated areas under each peak was calculated. Hitachi S4700 field emission scanning electron microscope (FE-SEM) operated at $5.0 \mathrm{kV}$ was used to image the surface and cross-section of the AlN bridges before and after wet etching.

Figure. 4.10 shows the surface scanning electron microscope (SEM) image of the AlN films grown on $\mathrm{Si}$ (111) substrate patterned with $\mathrm{SiO}_{x}$ stripes. It appears that the 
AlN film on $\mathrm{SiO}_{x}$ contains tilted grains, i.e. wurtzite grains, with the $\mathrm{c}$-axis at an angle to surface normal, typical of a polycrystalline films. From the SEM image of the crosssection in Figure 4.10 (b) it can be seen that the growth of AlN on Si is more ordered when compared to the AlN film on $\mathrm{SiO}_{\mathrm{x}}$.

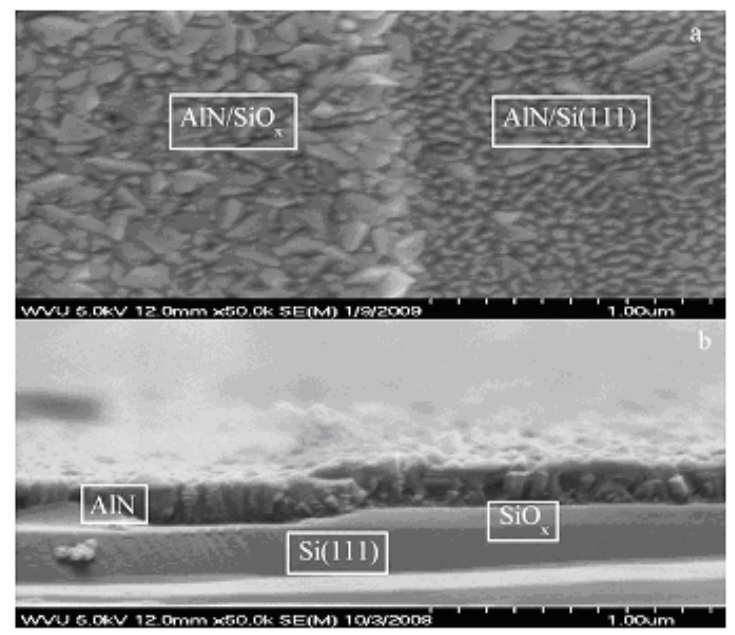

Figure 4.10: SEM images of the AIN film grown on $\mathrm{Si}$ (111) substrate patterned with $\mathrm{SiO}_{\mathrm{x}}$ stripes: (a) Surface (b) Cross-section.

This difference in growth can be attributed to the formation of an oxidized aluminum phase during the initial stages of the AlN film growth on $\mathrm{SiO}_{\mathrm{x}}$. The favorable reaction between oxygen and aluminum coupled with the amorphous nature of the PECVD deposited $\mathrm{SiO}_{\mathrm{x}}$ film lead to such a preferential growth of tilted $\mathrm{AlN}$ grains on $\mathrm{SiO}_{\mathrm{x}}$ [75], [76]. The AlN films on the substrate and the $\mathrm{SiO}_{x}$ layer were further characterized by micro FTIR transmission techniques. The FTIR absorption spectrum of a c-axis oriented AlN on $\mathrm{Si}$ (111) after Si background subtraction is shown in Figure 4.11. 


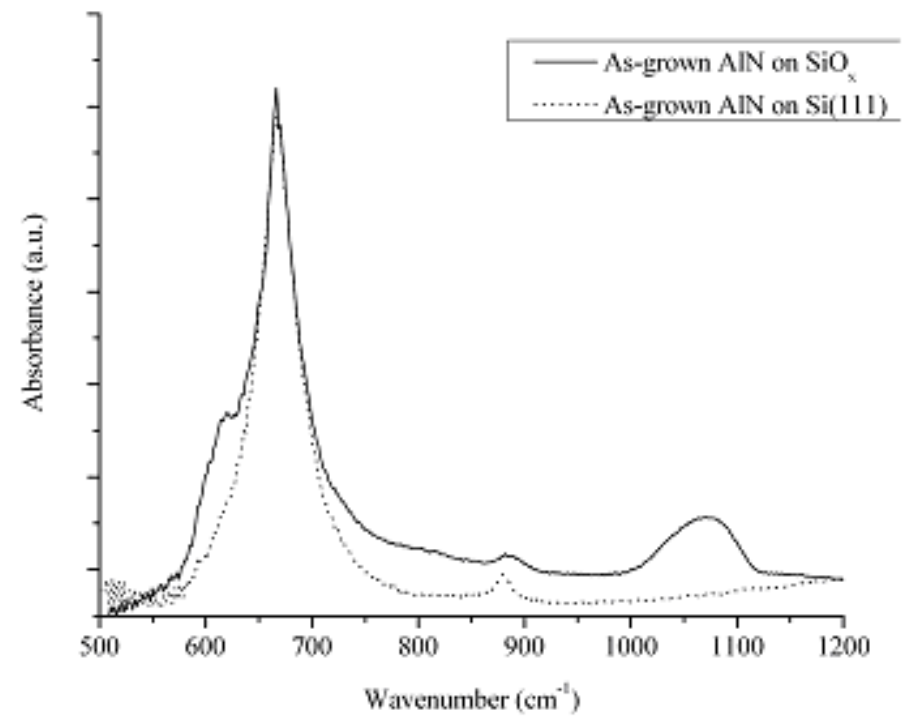

Figure 4.11: FTIR transmission spectrum of the AlN film on $\mathrm{Si}(111)$ and $\mathrm{SiO}_{\mathrm{x}}$.

The spectrum is dominated by a phonon absorption peak at $667 \mathrm{~cm}^{-1}$, corresponding to the c-axis oriented AlN film on the Si (111) substrate and this can be assigned to the transverse optical (TO) mode E1 (TO) of the AlN film [69]. As the transmission spectrum of the sample is at an oblique angle of incidence, the FTIR microscope setup also allows the observation of the longitudinal optical (LO) mode A1 (LO) in addition to the E1 (TO) mode in the absorption spectrum as shown in Figure 4.11 [72]. In comparison, the spectrum of $\mathrm{AlN} / \mathrm{SiO}_{\mathrm{x}}$ bilayer shows a broad band between $1000 \mathrm{~cm}^{-1}$ and $1100 \mathrm{~cm}^{-1}$ due to the asymmetric stretching mode of the oxygen atom in the $\mathrm{Si}-\mathrm{O}-\mathrm{Si}$ chain of amorphous $\mathrm{SiO}_{\mathrm{x}}$ [77]. The SEM images show a disordered AlN growth on $\mathrm{SiOx}$. The disordered growth led to the degradation in the crystal quality of $\mathrm{AlN}$ on $\mathrm{SiO}_{\mathrm{x}}$. A distinct shoulder appears to the left of the dominant peak in the AlN film grown on $\mathrm{SiO}_{\mathrm{x}}$. The appearance of the shoulder is related to the A1 (TO) mode of tilted wurtzite AlN grains in the AlN films on $\mathrm{SiO}_{\mathrm{x}}$ [70]. The presence of $\mathrm{A} 1$ (TO) modes is indicative of AlN grains with mixed orientation in which the c-axis is at an angle to the surface normal [70]. Further the onset of an absorption band around $885 \mathrm{~cm}^{-1}$ corresponding to the A1(LO) mode of wurtzite AlN [69] along with the E1 (TO) peak at $667 \mathrm{~cm}^{-1}$ in the $\mathrm{AlN} / \mathrm{SiO}_{\mathrm{x}}$ transmission spectrum implies that the AlN films predominantly contain a 
wurtzite phase [78]. An asymmetry and a shift also appear in the A1 (LO) mode related to the tilted AIN grains, providing a further indication that such grains are present [79]. The shift in the peak positions could be due to the residual stress variation in the AlN film on $\mathrm{Si}(111)$ and $\mathrm{AlN}$ film on $\mathrm{SiO}_{\mathrm{x}}$ stripes.

\subsubsection{Investigating the effect of buffered HF on AIN Micro-FTIR}

The samples were etched in buffered HF (10:1) solution for different times and FTIR transmission spectra were collected from the etched samples. Figure 4.12 shows the FTIR transmission spectra of AlN/SiOx after Si (111) background subtraction. From Figure 4.12 it can be seen that the width of the dominant peak decreases with increasing etch time.

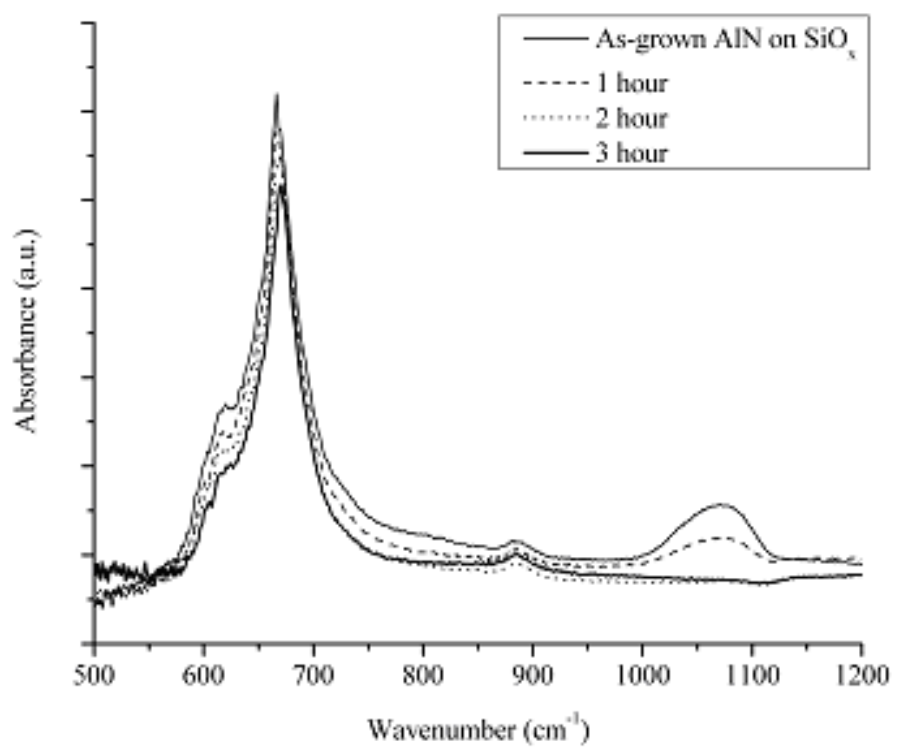

Figure 4.12: Variation in the FTIR transmission spectrum of $\mathrm{AlN} / \mathrm{SiO}_{\mathrm{x}}$ bilayer measured after etching the samples for different times in buffered $\mathrm{HF}(10: 1)$ solution.

The decrease in the width is a result of a change in the material properties of the AlN film on $\mathrm{SiO}_{\mathrm{x}}$ when etched. The SEM images of the $\mathrm{AlN} / \mathrm{SiO}_{\mathrm{x}}$ surface before and after etching from Figure 4.13 (a) and Figure 4.13 (b) indicate that the AlN film corrodes in buffered HF (10:1) solution. A review of the FTIR spectra from Figure 4.12 also shows a decrease 
in the intensity of the $\mathrm{SiO}_{\mathrm{x}}$ peak, which ultimately disappears after 2 hours of etching in buffered HF (10:1) solution.

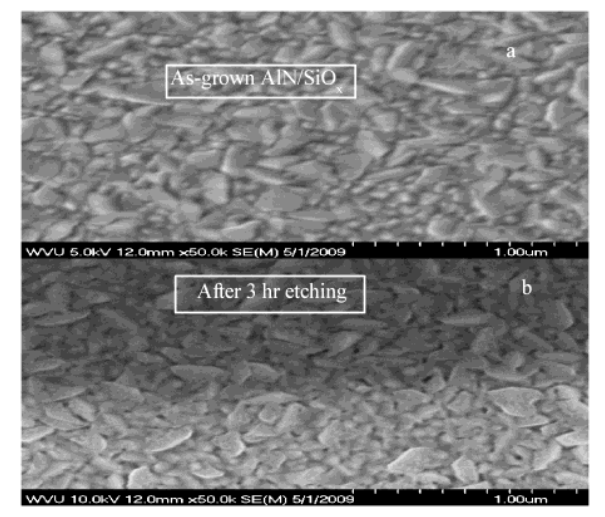

Figure 4.13: SEM surface images of AlN film on $\mathrm{SiO}_{x}$ : (a) As-grown (b) Etched in buffered HF (10:1) for 3 hours.

The wet etching mechanism that removes the underlying $\mathrm{SiO}_{\mathrm{x}}$ and forms channels several hundred micrometers in length within such a short time is unclear at this point, as the experimentally determined etch rate of $\mathrm{SiO}_{\mathrm{x}}$ in buffered $\mathrm{HF}$ (10:1) solution is only about $130 \mathrm{~nm} / \mathrm{min}$. On further etching the samples the absorbance spectrum shows a decrease in the width of the spectrum on the lower wavenumber side around the dominant (AIN) peak. Figure 4.14 shows the cross section of the films after 3 hours etching.

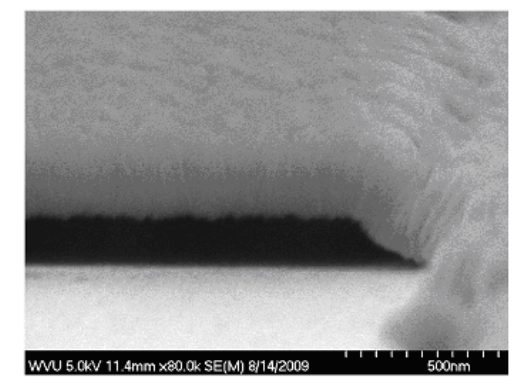

Figure 4.14: SEM cross section image of a suspended AIN structure after sacrificial layer etching 
The $\mathrm{SiO}_{\mathrm{x}}$ sacrificial was removed and in the process formed suspended AlN structures.

In order to quantify the observations, Lorentz functions were fitted to the spectra to obtain the peak position and the integrated area. Figure 4.15 shows that the Lorentz function fitted the FTIR transmission spectra of $\mathrm{AlN} / \mathrm{SiO}_{\mathrm{x}}$ bilayer in the region between $500 \mathrm{~cm}^{-1}$ and $850 \mathrm{~cm}^{-1}$.

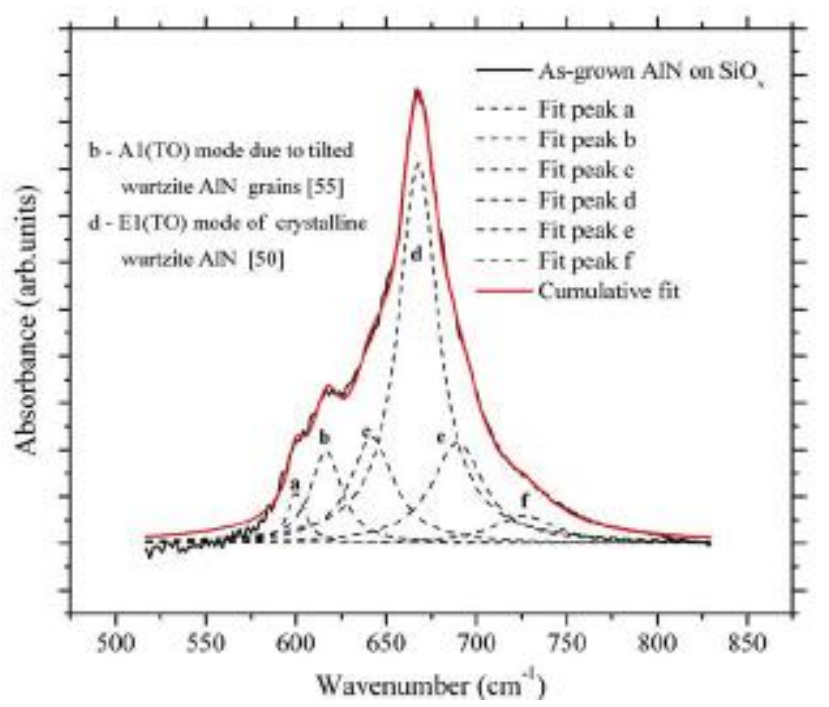

Figure 4.15: FTIR transmission spectrum of as-grown AlN film on $\mathrm{SiO}_{\mathrm{x}}$. Dashed lines show deconvoluted Lorentz functions.

The deconvoluted spectrum reveals several peaks. Peaks b and d correspond to the A1 (TO) and E1 (TO) modes of wurtzite AlN films respectively. The origin of the other peaks namely a, e and f could possibly be due to the formation of oxidized AlN [80], nonstoichiometric AlN [81] and nanocrystalline AlN [82]. Peak c could correspond to cubic AlN or non-stoichiometric AlN. Similarly the FTIR transmission spectra of AlN on $\mathrm{SiO}_{\mathrm{x}}$ etched for 1, 2, and $3 \mathrm{~h}$ were quantified using Lorentz functions to obtain the integrated areas. A plot of the integrated areas under A1 (TO) and E1 (TO) modes of the AlN film on $\mathrm{SiO}_{\mathrm{x}}$ as a function of etching time is shown in Figure 4.16. 


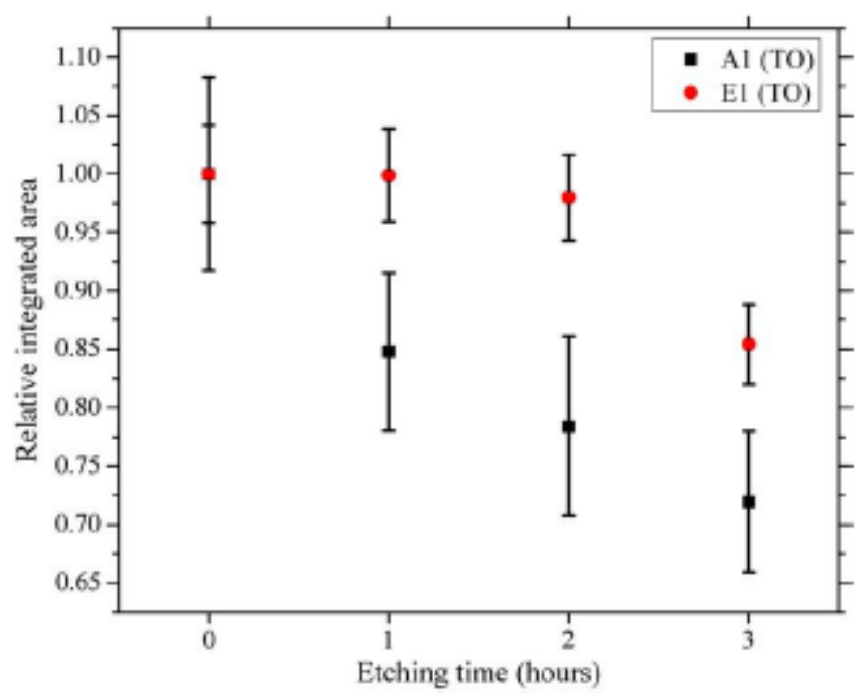

Figure 4.16: Ratio of the integrated area of the vibrational modes of etched samples with respect to unetched samples for $\mathrm{AlN}$ film on $\mathrm{SiO}_{\mathrm{x}}$.

The integrated areas under A1 (TO) and E1 (TO) were normalized with respect to the integrated areas of the vibrational modes A1 (TO) and E1 (TO) of as-grown AlN films on $\mathrm{SiO}_{\mathrm{x}}$ respectively.

The A1 (TO) mode representing the tilted AlN grains showed a reduction in the relative integrated area after $1 \mathrm{~h}$ of etching. This shows that the mixed crystallographic phases in the film are reduced to some extent. No significant reduction was observed after 2 and $3 \mathrm{~h}$ of etching. The relative integrated area under the E1 (TO) mode shows a reduction after a 3 hour etching. From the etching trends it can be concluded that the AlN grains corroded in buffered HF when exposed for extended time leading to a change the material properties of the AlN air bridges. The mixed interphases co-exist with caxis oriented crystals. After etching, the non c-axis oriented domains are mostly etched away. Prior work showed that the integrated area representing the tilted AlN grains is proportional to the amount of tilted grains in the film and the presence of the grains leads to a degradation in the piezoelectric properties [70]. As the $\mathrm{AlN}$ film on $\mathrm{SiO}_{\mathrm{x}}$ was shown to contain tilted grains prior to post processing, it is 
possible that the as-grown AlN films on $\mathrm{SiO}_{x}$ possesses inferior piezoelectric properties though electrical characterization is required to determine the piezoelectric properties. However, post processing the films by wet etching led to a reduction in the amount of tilted AlN grains which could improve the piezoelectric properties of the AlN films. Further work is required to study the effect of wet etching on the material properties of the AlN films grown on $\mathrm{SiO}_{\mathrm{x}}$.

\subsection{Profile of $\mathrm{SiO}_{\mathrm{x}}$ sacrificial layer after etching in buffered $\mathrm{HF}$}

FTIR analysis shows that AIN suspended structures with lengths of few hundred microns were found within 3 hours of etching. Amorphous $\mathrm{SiO}_{x}$ etches istropically in buffered HF (10:1) solution. The experimentally determined etch rates of $\mathrm{SiO}_{\mathrm{x}}$ deposited by PECVD are between $130-400 \mathrm{~nm} / \mathrm{min}$ depending on the deposition conditions such as temperature of the substrate. Prior to etching the sacrificial layer the samples are cleaved to expose the sacrificial layer. The etching of the $\mathrm{SiO}_{\mathrm{x}}$ layer is therefore expected to proceed laterally. But based on the etch rate of $\mathrm{SiO}_{\mathrm{x}}$ it is highly unlikely that channels of a few millimeters can be fabricated in such a short time. FTIR measurements have shown that the as-grown AlN films on $\mathrm{SiO}_{\mathrm{x}}$ etch in buffered $\mathrm{HF}$ solution. Therefore, in order to determine the etching profile of the sacrificial layer for the dimension of $\mathrm{SiO}_{\mathrm{x}}$ under consideration hard baked photo resist was used to mask the $\mathrm{SiO}_{\mathrm{x}}$ layer. Photo resist is a polymer and is known to be chemically stable in buffered HF solution. The flow process is shown Figure 4.17.

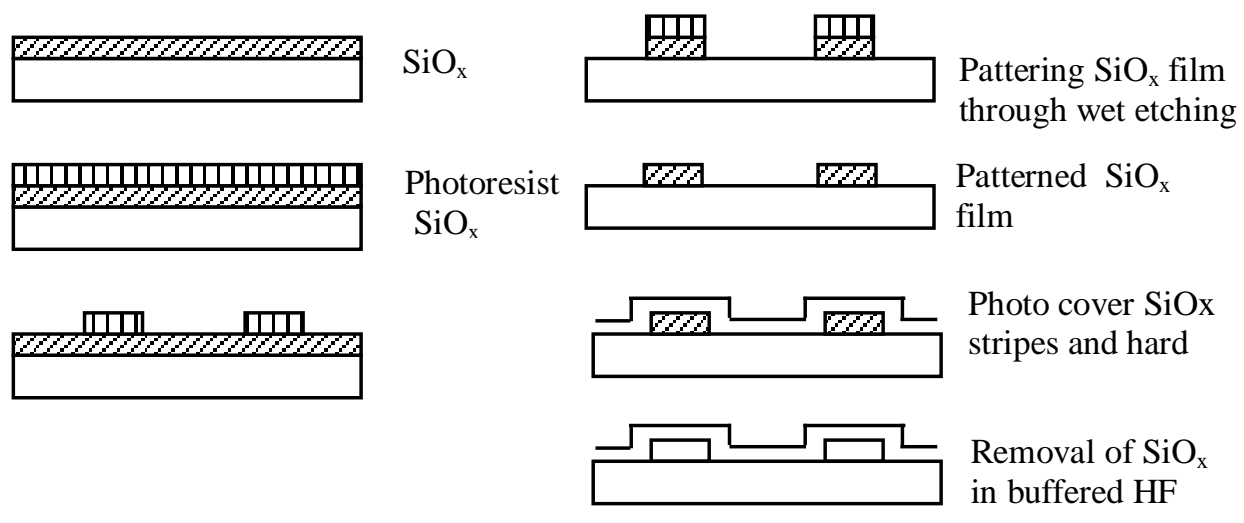

Figure 4.17: Process flow for investigating the etch profile of $\mathrm{SiO}_{\mathrm{x}}$ 
$\mathrm{SiO}_{\mathrm{x}}$ films were deposited by PECVD techniques on a silicon substrate. The film was patterned into stripes with varying widths between $40-100 \mu \mathrm{m}$ using standard lithography process and wet etching. AZ5214 photo resist was spun uniformly on the patterned sample yielding a photo resist thickness of $1.4 \mu \mathrm{m}$. The sample was then baked at $110^{\circ} \mathrm{C}$ for 30 minutes to harden the photo resist as a mask. Baking also enhances the adhesion of the photo resist to the sacrificial layer. The samples were cleaved into several pieces and to expose the underlying $\mathrm{SiO}_{\mathrm{x}}$ layer. The samples were etched for different times in buffered HF solution. Finally the photo resist was stripped in a photo resist stripper to reveal the etch profile of the $\mathrm{SiO}_{\mathrm{x}}$ layer. Shown in Figure 4.18 are some etch profiles for different widths and etching times. The thickness of $\mathrm{SiO}_{\mathrm{x}}$ used for the experiments is around $920 \mathrm{~nm}$.

$\mathrm{SiO}_{2}$ width $40 \mu \mathrm{m}$

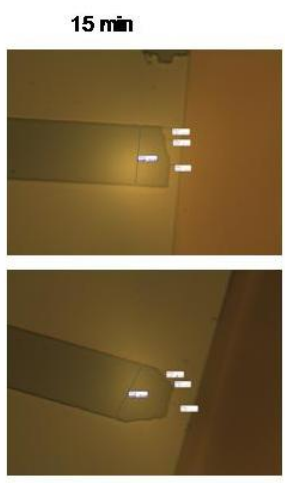

$25 \mathrm{~min}$
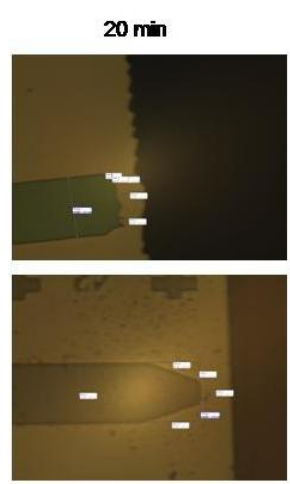

$30 \mathrm{~min}$

$\mathrm{SiO}_{2}$ width $70 \mu \mathrm{m}$

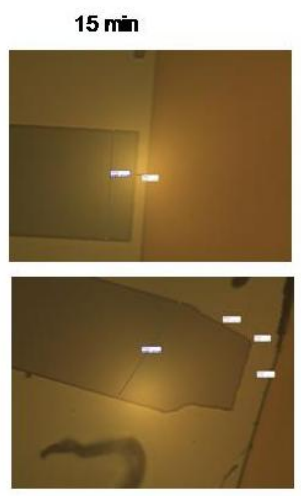

$25 \mathrm{~min}$

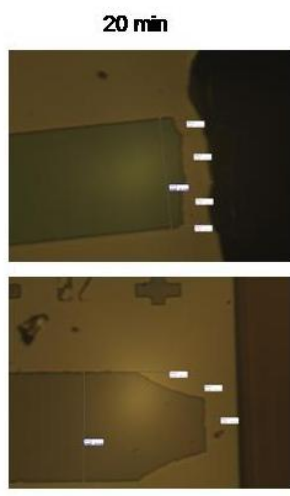

$30 \mathrm{~min}$

Figure 4.18: Etch profile of SiOx after removal of photo resistmask

It is clear from the optical images that the etch profile is not uniform. The profile tapers off with increasing etch time. However the etch mechanism for the AlN suspended structure is expected to be different due to the fact that the AlN films corrode in buffered HF solution. The etching mechanism was investigated by using Micro-Raman techniques. 


\subsection{Effect of buffered HF on AIN films grown on $\mathrm{SiO}_{\mathrm{x}}$ - Micro Raman}

The as-grown samples were cleaved to expose the sacrificial layer and were then etched in buffered HF (10:1) solution. At distances of tens of microns to hundreds of microns from the cleaved edges the $\mathrm{AlN}$ film on $\mathrm{SiO}_{\mathrm{x}}$ displayed an apparent change in the texture when inspected under an optical microscope. The optical images of $\mathrm{AlN} / \mathrm{SiO}_{\mathrm{x}}$ bilayers on $\mathrm{GaN}$ and $\mathrm{Si}(111)$ templates after a $30 \mathrm{~min}$ wet etching are shown in figure 4. Regions of discoloration are clearly evident from Figure 4.19. The lateral etching of the sacrificial layer initiated from the cleaved edges could not have caused the discoloration, as the measured etch rate of $\mathrm{SiO}_{\mathrm{x}}$ was only $130 \mathrm{~nm} / \mathrm{min}$. A similar change in texture was observed for all the widths. It appears that the $\mathrm{AlN}$ films on $\mathrm{SiO}_{\mathrm{x}}$ corroded in buffered $\mathrm{HF}$ solution. Henceforth the AlN films on $\mathrm{SiO}_{\mathrm{x}}$ will be addressed as $\mathrm{AlN}$ bridges.

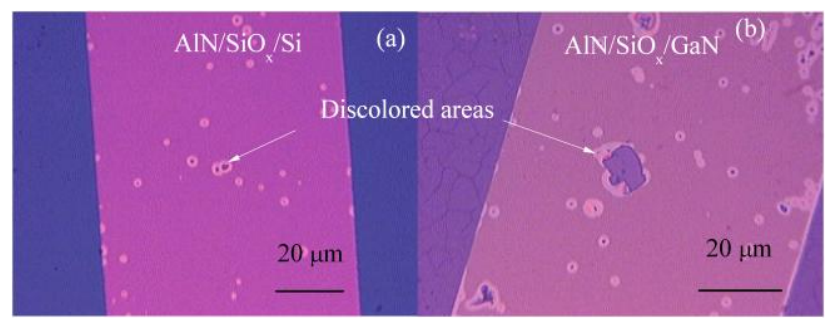

Figure 4.19: Optical images of the AlN bridges after etching in buffered HF (10:1) solution for $30 \mathrm{~min}$ (a) $\mathrm{Si}$ (111) (b) GaN.

The Raman spectra collected from the discolored regions revealed peaks within the range $600 \mathrm{~cm}^{-1}$ to $700 \mathrm{~cm}^{-1}$ and a reduction in the background noise as shown in Figure 4.20. It has been shown that the as-grown $\mathrm{AlN}$ films on $\mathrm{SiO}_{\mathrm{x}}$ are in a predominantly wurtzite phase and that the average quality of AlN bridges improved as a result of wet etching in buffered HF solution [83]. The micro-FTIR spectra collected over an area containing several such discolored regions shows a gradual removal of the sacrificial layer as well due to the wet etching process. The observed discoloration of the AlN film on $\mathrm{SiO}_{\mathrm{x}}$ is therefore due to the change in the material properties of the AlN film and also results from the etching of the underlying sacrificial layer. 

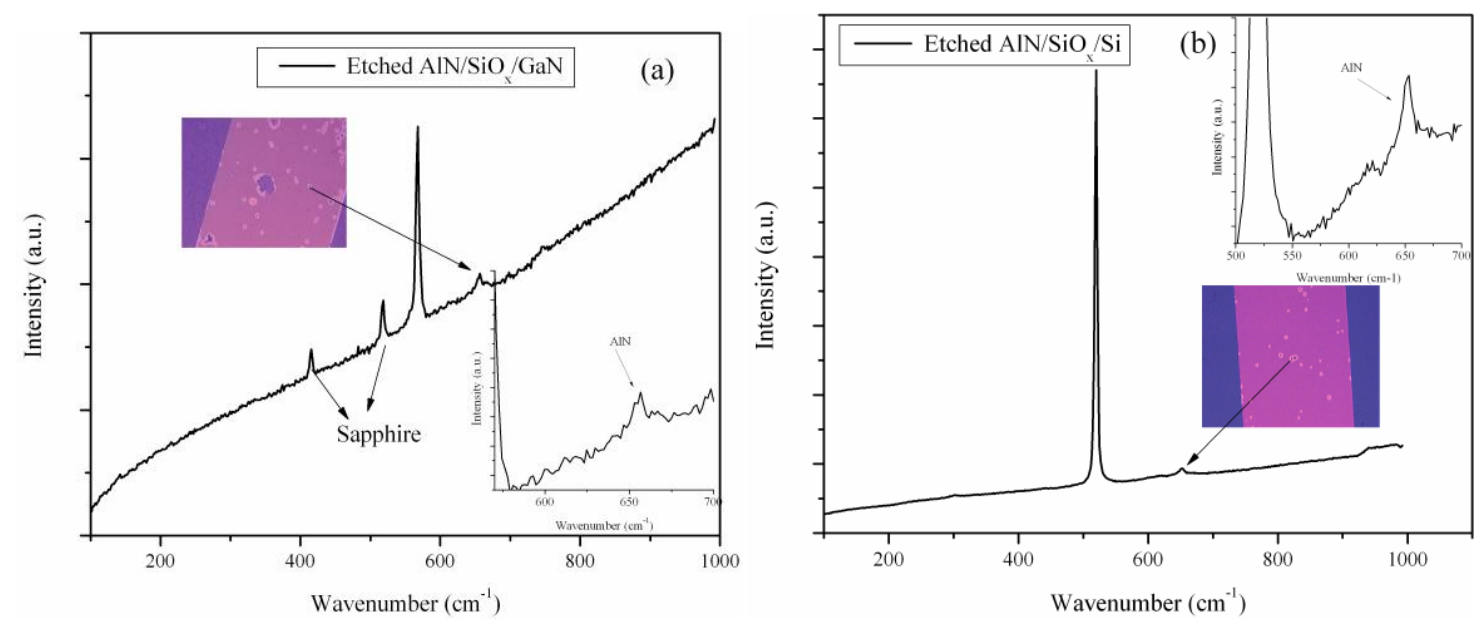

Figure 4.20: Raman spectra collected from the discolored regions of AlN bridges after etching in buffered HF solution (10:1) showing the AlN E2 (high) peak (a) GaN (b) Si (111).

The inset spectra of Figure 4.20 show a prominent peak within the range $600 \mathrm{~cm}^{-1}$ to 700 $\mathrm{cm}^{-1}$ indicating the presence of wurtzite AlN. The spectra collected from the bridges on $\mathrm{GaN}$ also show the emergence of sapphire peaks after etching. Which indicates a change in transmission properties of the AlN film.

The samples were etched for extended time to completely remove the underlying sacrificial layer from across the width and length thereby forming an air-bridge. SEM image of an air-bridge is shown in Figure 4.21.

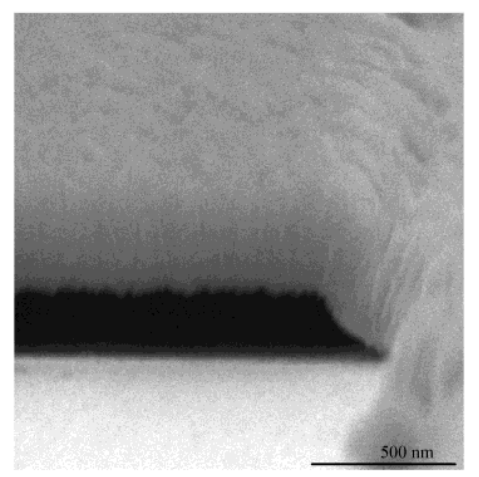

Figure 4.21: SEM cross-section image of an AlN air-bridge after sacrificial layer etching. 
The optical images of AlN air-bridges are shown as inset images in Figure 4.22 (a) and Figure 4.22 (b). Raman spectra were collected from across the width of the airbridges. The spectra obtained at uniformly spaced points along the line scan were deconvoluted by fitting Lorentz functions to identify the peaks as shown in Figure 4.31(a). The deconvoluted spectra show a major peak between $654 \mathrm{~cm}^{-1}$ and $656 \mathrm{~cm}^{-1}$ along with a relatively weak peak at $669 \mathrm{~cm}^{-1}$ corresponding to E2 (high) and E1 (TO) mode of wurtzite AlN crystals respectively.

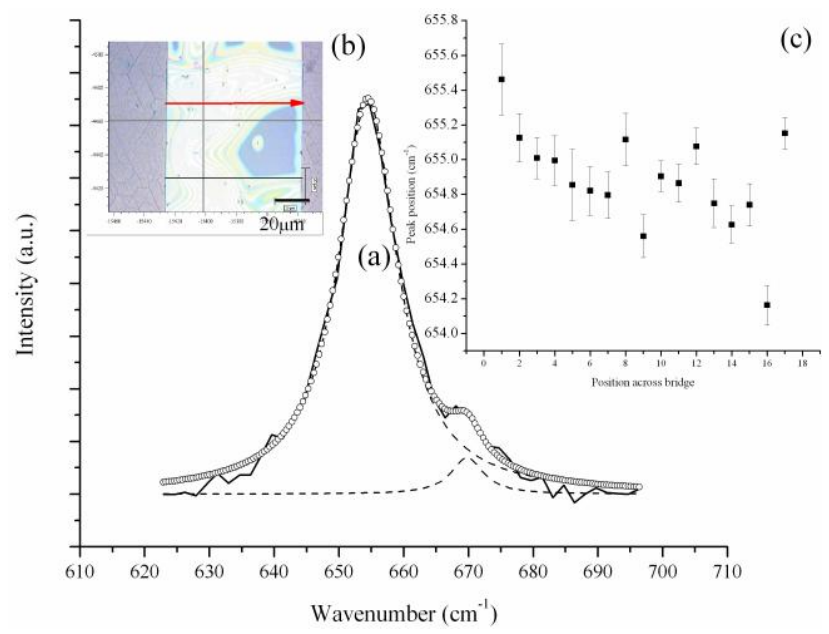

Figure 4.22: AlN air-bridge $80 \mu \mathrm{m}$ wide on GaN (a) Raman spectra after peak separation (b) Direction of line scan indicated by red arrow from left to right (c) peak position of E2 (high) mode along the line scan.

It is has been experimentally shown that the Raman spectra of AlN crystals, obtained under polarized conditions, consisting of a relatively weak E1 (TO) mode and a dominant E2 (high) mode have the c-axis oriented preferentially along the axis perpendicular to the surface [84]. Based on the observations from the deconvoluted Raman spectra in Figure 4.22(a), it can be concluded that the AIN air-bridges are preferentially c-axis oriented. Also the quasi modes corresponding to misoriented AlN domains were not detected in the Raman spectra of the air-bridges during the peak fit process. The E2 (low) mode of the AlN films grown on GaN and Si (111) templates could not be observed due to thickness limitations effects and, naturally was not detected in the Raman spectra of the AlN air- 
bridges. The E2 (high) peak position for bulk AlN was reported to be around $655 \mathrm{~cm}^{-1}$ [84] and the stress free peak position can vary due to the presence of impurities such as oxygen [85], [86]. Based on the peak positions across the width shown in Figure 4.22(c) it can be concluded that the AIN air-bridges after the removal of the sacrificial layer possess a low residual stress due to stress relaxation. AlN air-bridges of widths $90 \mu \mathrm{m}$, $100 \mu \mathrm{m}$ and $110 \mu \mathrm{m}$ fabricated and characterized using similar techniques also exhibited a preferentially c-axis orientation and low residual stress characteristics.

Similarly Raman spectra were collected from AlN air-bridges fabricated on $\mathrm{Si}$ (111) substrates. As shown in Figure 4.23(b) the deconvoluted spectrum revealed a dominant E2 (high) peak and a very weak peak at $669 \mathrm{~cm}^{-1}$ thereby implying that the films were also preferentially c-axis oriented. Contributions from the silicon substrate to the intensity of the AlN E1 (TO) mode cannot be ruled out as the Raman modes obtained from a bare Silicon substrate were found to exist around $670 \mathrm{~cm}^{-1}$. Raman modes from Si substrate also occur between $600 \mathrm{~cm}^{-1}$ and $620 \mathrm{~cm}^{-1}$. Also the peak positions shown in Figure 4.23 (c) indicate that the AlN air-bridges after the removal of the sacrificial layer possess a low residual stress. AlN air-bridges having widths $90 \mu \mathrm{m}, 100 \mu \mathrm{m}$, and 110 $\mu \mathrm{m}$ were found to exhibit similar characteristics such as preferential c-axis orientation and stress relaxation.

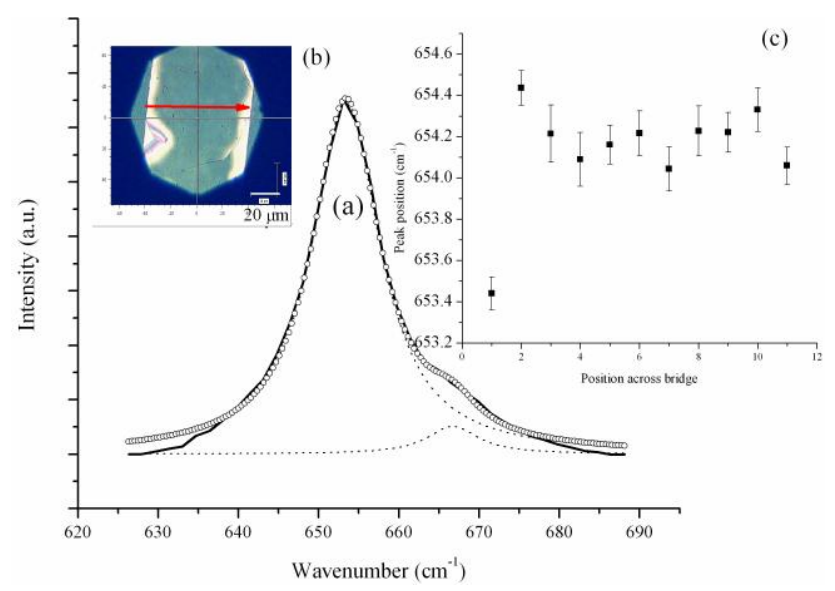

Figure 4.23: AlN air-bridge $80 \mu \mathrm{m}$ wide on Si (111) (a) Raman spectra after peak separation (b) Direction of line scan indicated by red arrow from left to right (c) peak position of E2 (high) mode along the line scan. 
Typically the full width at half maximum (FWHM) value of the E2 (high) mode is an indication of the crystal quality of the films [86]. The lowest FWHM reported for bulk AlN is $3 \mathrm{~cm}^{-1}$ [87] and for amorphous AlN $50 \mathrm{~cm}^{-1}$ [88]. A comparison of the average FWHM values obtained from AlN air-bridges grown on different substrates, for the purposes of this work, is shown in Table 4.2. These intermediate values indicate that the AlN films still contain non negligible crystal defect concentrations.

Table 4.2: FWHM values of E2 (high) peaks of AlN air-bridges.

\begin{tabular}{cc}
\hline Sample & FWHM of AlN E2(high) $\mathrm{cm}^{-1}$ \\
\hline AlN air-bridge on GaN & 12.20 \\
AlN air-bridge on Si(111) & 9.86
\end{tabular}

However, a variation exists in the FWHM values of the AlN air-bridges fabricated on GaN/Sapphire and Si (111) templates even though the growth conditions were similar. The FWHM of AlN air-bridges on GaN is higher when compared to the AlN air-bridges fabricated on Si (111). The difference in the FWHM could be due to the variation in the crystal quality of the as-grown AlN films on $\mathrm{SiO}_{\mathrm{x}}$. The $\mathrm{SEM}$ surface images of $\mathrm{AlN} / \mathrm{SiO}_{\mathrm{x}}$ shown in Figure 4.15 clearly indicate a difference in the surface texture of the AlN films on $\mathrm{SiO}_{\mathrm{x}}$ grown on $\mathrm{GaN}$ and $\mathrm{Si}$ templates, pointing to crystal quality variation. This implies a substrate induced effect on the crystal quality despite the sacrificial layer being amorphous. However, due to the limitations of the micro-Raman instrument setup used for the experiments this effect could not be quantified for the as-grown samples. Another factor contributing to the observed difference in the FWHM values could be due the wet etching process that changed the material properties of the AlN air-bridges.

An important question arises as to why the AlN air-bridges are preferentially caxis oriented despite the amorphous structure of the underlying sacrificial layer. These results can be explained through the texture development of polycrystalline films on amorphous surfaces [89] as shown in Figure 4.24. 


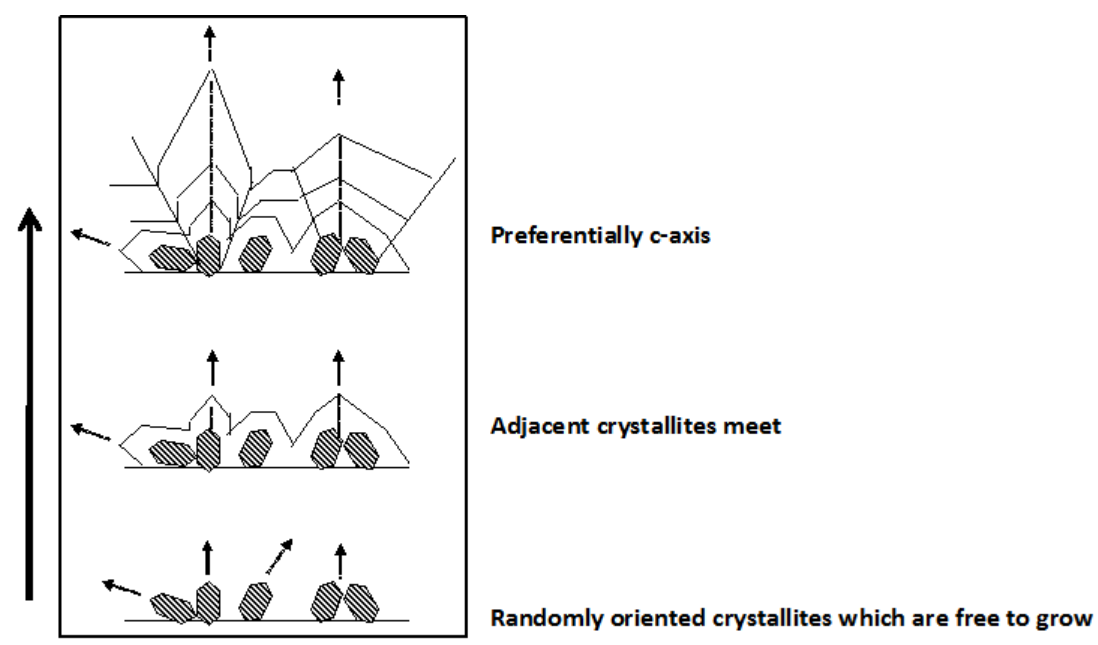

Figure 4.24: Texture growth model for thin films on amorphous substrates [89]

The growth process starts with formation of randomly oriented crystallites which are free to grow. In the later stages adjacent crystallites meet and form intercrystalline impingement boundaries. In the subsequent steps because the growth rate depends on the crystalline directions, it appears that c-axis grows preferentially over other crystallographic orientations. Therefore, on an average the c-axis oriented crystallites dominate the non c-axis oriented domains. During the wet etching process the observed discoloration of the AIN air-bridges could be due to the removal of mixed interphases coexisting with preferentially c-axis oriented crystals. After etching, the non c-axis oriented domains are mostly etched away and the Raman spectra resemble those of wurtzite AlN originating from the remaining crystallites in the film. This process could lead to the appearance of Raman peaks corresponding to wurtzite AlN.

\subsubsection{Hydrolysis of AIN in aqueous solutions:}

It is known that AIN undergoes hydrolysis in water base solutions [90] .

$$
\mathrm{AlN}+\mathrm{H}_{2} \mathrm{O} \rightarrow \mathrm{AlOOH}+\mathrm{NH}_{3}
$$

Prolonged exposure to humid conditions results in the formation of aluminum hydroxide

$$
\mathrm{AlOOH}+\mathrm{H}_{2} \mathrm{O} \rightarrow \mathrm{Al}(\mathrm{OH})_{3}
$$


Aluminum hydroxide is amphoteric in nature. An amphoteric substance reacts with both acids and bases. In the presence of a base the substance acts as an acid and in the presence of an acid the substance acts as a base [91].

Polycrystalline AlN films undergo significant hydrolysis due to the presence of grain boundaries. Depending on the crystal structure the hydrolysis can extend to a significant depth from the surface as shown in Figure 4.25. As the AlN films on $\mathrm{SiO}_{\mathrm{x}}$ exhibit polycrystalline growth the films are expected to undergo significant hydrolysis.

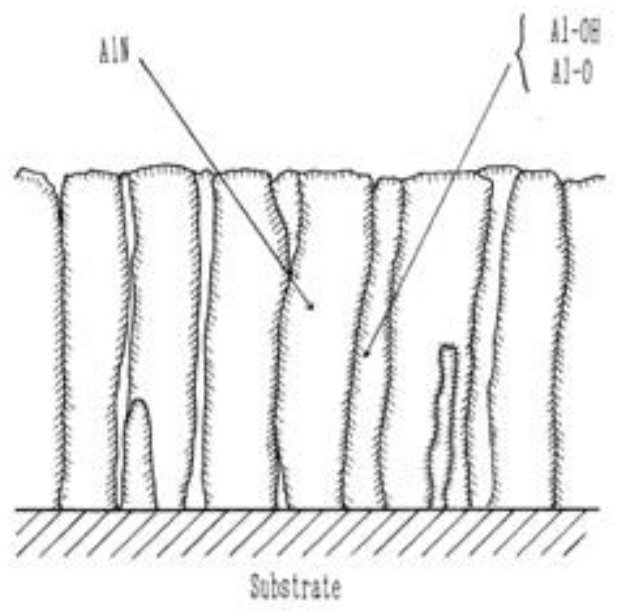

Figure 4.25: Schematic diagram of oxidation state of aluminum nitride thin film [92]

AlN were etched in buffered HF (10:1) solution, which is an aqueous solution. In the presence of water the films undergo hydrolysis forming aluminum hydroxide. But buffered HF is an acidic solution and aluminum hydroxide acts as base in its presence. The reaction between an acid and base results in the formation of salt as a by product, which dissolves in water. The reaction proceeds progressively dissolving AlN in the process and changing the material properties. The observed discoloration of the AlN films is a result of a change in the material properties of the AlN films. The etching process appears to create alternate path ways from the AlN surface, which reach out to the underlying $\mathrm{SiO}_{\mathrm{x}}$ layer. This process aids in the etching of the $\mathrm{SiO}_{\mathrm{x}}$ layer thereby creating suspended AlN structures in a short time. Shown in Figure 4.26 are the optical images of AlN air-bridges fabricated on $\mathrm{Si}$ (111) and GaN/sapphire templates. After wet 
etching the AlN air-bridges exhibit severe cracking as show in Figure 4.26. But the airbridges fabricated on $\mathrm{GaN}$ apparently do not exhibit cracks but display a creep pattern.
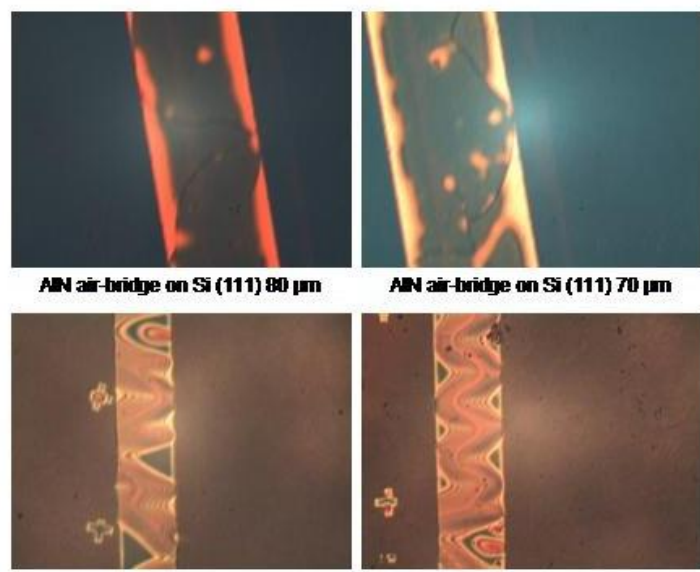

AN air-biutge on Si (111) $70 \mathrm{~m}$

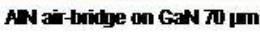

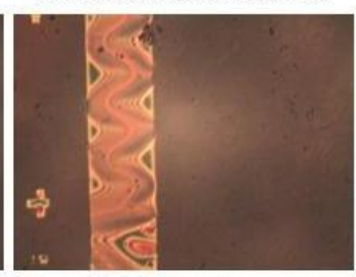

AN ai-huidge on GaN 80 pum

Figure 4.26: Optical images of full eteched AlN air bridges on Si (111) and GaN/Sapphire template

\subsection{Residual stress in AIN air-bridges due to thermal mismatch:}

Residual stress in a thin film is primarily composed of intrinsic stress and extrinsic stresses. Intrinsic stresses develop during the thin film growth while extrinsic stresses are imposed on the thin film by external factors such as temperature. Common forms of extrinsic stresses include thermal stress. Thermal stress originates due to the difference in the coefficient of thermal expansion between the thin film and substrate. Intrinsic stresses include stress due to misfit dislocations, inclusion of impurities, difference in lattice mismatch between the thin film and substrate as shown in Figure 4.27. 

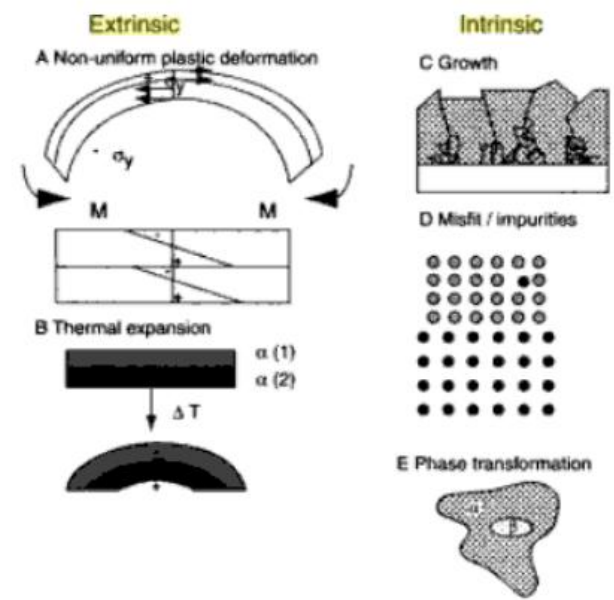

Figure 4.27: Examples of intrinsic and extrinsic stress in thin films [93]

For the $\mathrm{SiO}_{\mathrm{x}}$ films deposited on $\mathrm{Si}$ substrate thermal stress is expected to dominate the residual stress in the $\mathrm{SiO}_{\mathrm{x}}$ films, due the non crystalline nature of $\mathrm{SiO}_{\mathrm{x}}$. Thermal stress arises because the deposition temperature of thin films is usually above room temperature in a CVD processes. As the sample cools down to the room temperature from the deposition temperature, the film experiences a residual compressive or tensile stress, due to the difference in the thermal expansion coefficient of the film and the substrate.

$$
\begin{aligned}
& \alpha_{\mathrm{f}} \text { : Thermal expansion coefficient of the film } \\
& \alpha_{\mathrm{s}} \text { : Thermal expansion coefficient of the substrate }
\end{aligned}
$$

Considering a film which is thin compared to the thickness of the substrate, as an approximation the thin film is expected to contract by the same amount as the substrate. A thin film attached rigidly to a substrate experiences an in-plane stress. Stresses normal to the film surface are equal to zero. If the in-plane stress components are equal then the in-plane stress is called biaxial stress. Assuming the linear thermal expansion coefficient of the film and substrate does not vary with temperature, the average in-plane thermal mismatch strain in the film is given by [94]:

$$
\left.\varepsilon_{f, \text { mismatch }}=\alpha_{f}-\alpha_{s}\right\rangle T
$$

Where , $\Delta T$ is the difference between the temperature of deposition of the film $\mathrm{T}_{\mathrm{dep}}$ and room temperature $T_{r}$ 
The biaxial stress in the thin film is given by :

$$
\alpha_{f, \text { mismtach }}=\left(\frac{E}{1-v}\right)_{f} \varepsilon_{f, \text { mismatch }}
$$

E- Young's modulus of the film

$v$ - Poisson's ratio of the thin film

If the thermal expansion coefficient of the film is smaller than that of the substrate the film is in compressive stress. If the thermal expansion coefficient of the film is more than the substrate the film after cooling down to the room temperature is in tensile stress as shown in Figure 4.28.

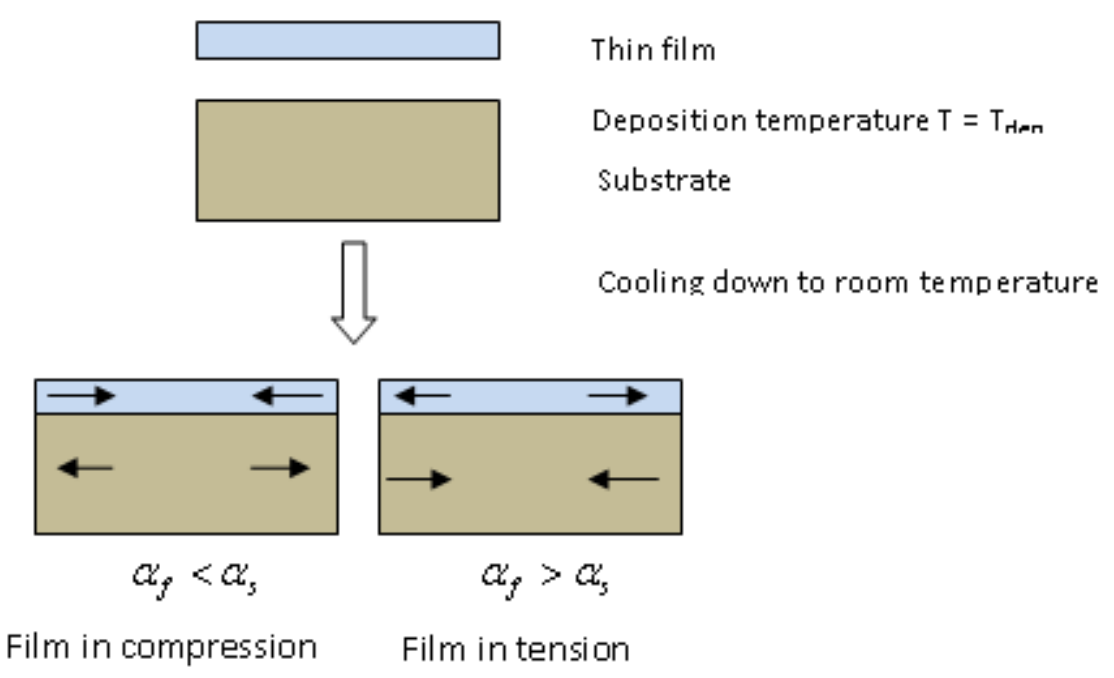

Figure 4.28: Residual stress in thin film due to mismatch of the coefficient of thermal expansion between the film and the substrate

Table 4.3 lists the mean coefficient thermal expansion values of the materials that were used to fabricate the AlN air-bridges. 
Table 4.3: Mean thermal expansion coefficients of materials used to fabricate AlN airbridges

\begin{tabular}{|l|l|}
\hline Material & $\begin{array}{l}\text { Thermal expansion coefficient } \\
\text { (in-plane) } \times 10^{-6}{ }^{\circ} \mathrm{C}\end{array}$ \\
\hline Silicon & $3.57[95]$ \\
\hline Sapphire & $7.28[95]$ \\
\hline Silicon oxide & 0.5 \\
\hline GaN & $4.997[96]$ \\
\hline AlN & $5.27[95]$ \\
\hline
\end{tabular}

Table 4.4 lists the residual stress due to thermal mismatch in thin films deposited on GaN/sapphire and silicon substrates based on the values given in Table 4.2.

Table 4.4: Residual stress in $\mathrm{SiO}_{\mathrm{x}}$ on various substrates at room temperature

\begin{tabular}{|l|l|}
\hline Multilayers & $\begin{array}{l}\text { Residual stress in } \mathrm{SiO}_{\mathrm{x}} \text { due } \\
\text { to thermal mismatch }\end{array}$ \\
\hline $\mathrm{SiO}_{\mathrm{x}}(130 \mathrm{~nm}) / \mathrm{Si}(350 \mu \mathrm{m})$ & Compressive \\
\hline $\mathrm{SiO}_{\mathrm{x}}(130 \mathrm{~nm}) / \mathrm{Sapphire}(450 \mu \mathrm{m})$ & Compressive \\
\hline $\mathrm{GaN}(2 \mu \mathrm{m}) / \mathrm{Sapphire}(450 \mu \mathrm{m})$ & Compressive \\
\hline $\mathrm{SiO}_{\mathrm{x}}(130 \mathrm{~nm}) / \mathrm{GaN}(2 \mu \mathrm{m}) / \mathrm{Sapphire}(450 \mu \mathrm{m})$ & Compressive \\
\hline
\end{tabular}

The thermal expansion coefficient of $\mathrm{SiO}_{\mathrm{x}}$ is lower than the thermal expansion coefficient of $\mathrm{Si}$ and sapphire. This leads to a negative value in the biaxial stress in $\mathrm{SiO}_{\mathrm{x}}$, therefore the film is under compressive stress on both $\mathrm{Si}$ and sapphire substrates. The GaN films grown on the sapphire substrate had an intermediate low quality AlN buffer layer of 30 $\mathrm{nm}$ thickness. Due to the non-crystalline AlN buffer layer there is no residual stress due to lattice mismatch between $\mathrm{GaN}$ and sapphire. Further, the low crystal quality of the buffer layer ensures that there no residual stress due to lattice mismatch between AIN and $\mathrm{GaN}$. The residual stress in the GaN film when cooled down to the room temperature from the growth temperature is therefore compressive.

The thicknesses of the substrates considered in this work are much higher than the thin films thicknesses. Therefore in a two and three layer heterostructure consisting of 
AlN $(300 \mathrm{~nm}) / \mathrm{SiO}_{\mathrm{x}}(130 \mathrm{~nm}) / \mathrm{Si}(350 \mu \mathrm{m})$ and $\mathrm{AlN}(300 \mathrm{~nm}) / \mathrm{SiO}_{\mathrm{x}}(130 \mathrm{~nm}) / \mathrm{GaN}(2$ $\mu \mathrm{m}) /$ Sapphire $(450 \mu \mathrm{m})$ respectively the individual layers are expected to contract by the same amount as the substrate. Due to the amorphous nature of $\mathrm{SiO}_{\mathrm{x}}$ no residual stress due to lattice mismatch exists between $\mathrm{AlN}$ and $\mathrm{SiO}_{\mathrm{x}}$.

\subsubsection{Residual stress in $\mathrm{AIN} / \mathrm{SiO}_{\mathrm{x}} / \mathrm{Si}$}

Table 4.3 shows that the thermal expansion coefficient of $\mathrm{AlN}$ is higher than that of Si. Due this difference in the thermal expansion coefficients the AlN films on $\mathrm{SiO}_{\mathrm{x}}$ are expected to have a tensile residual stress at room temperature. Figure 4.29 shows the E1 (TO) peak position of AlN as a function of etching time. The position of the E1 (TO) mode indicates the type of residual stress present in AlN, with a stress free value for AlN at $670 \mathrm{~cm}^{-1}$ [97]. Figure 4.29 shows that the E1 (TO) peak position for the as-grown AlN film on $\mathrm{SiO}_{\mathrm{x}}$ is at $667 \mathrm{~cm}^{-1}$. This value indicates that the AlN films possess tensile residual stress as predicted 4.6. When the sacrificial layer is etched,with an increase in the etching time Figure 4.29 shows that the E1 (TO) peak moves towards the stress free reaching at value $669.8 \mathrm{~cm}^{-1}$ at 3 hours. However the AlN air-bridges appear fractured after etching for 3 hours as shown in Figure 4.26. The fracture of the AlN air-bridges occurs when the tensile stress exceeds the fracture limit of the thin film.

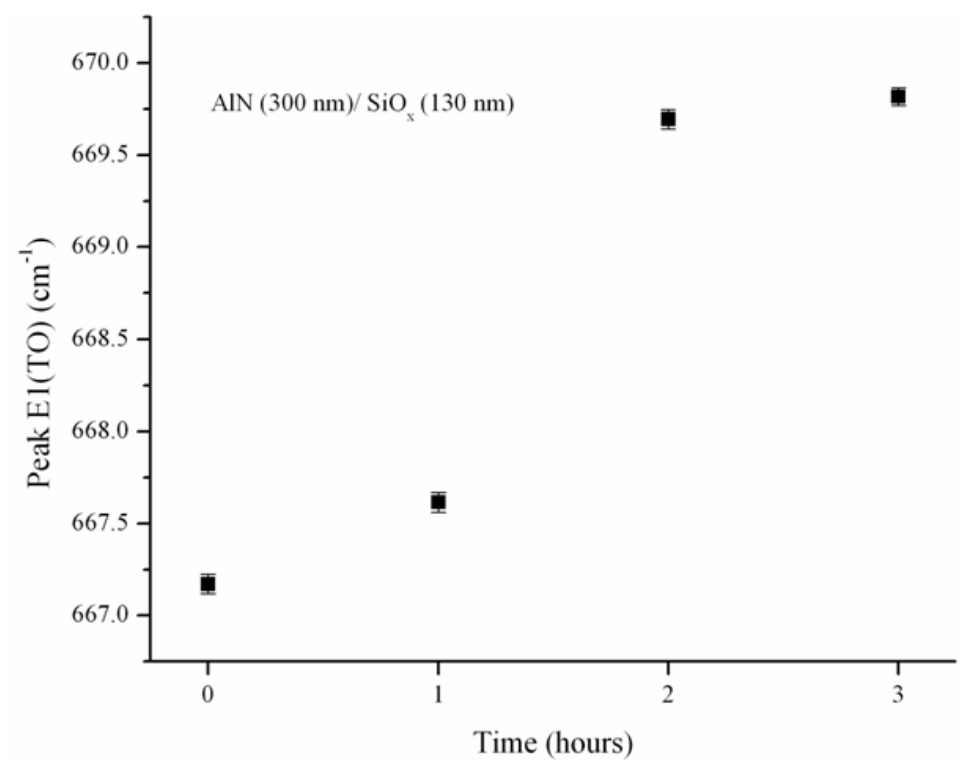

Figure 4.29: Peak position of the E1 (TO) mode of AlN films on $\mathrm{SiO}_{\mathrm{x}}$ as a function of etching time 


\subsubsection{Residual stress in $\mathrm{AlN} / \mathrm{SiO} \mathrm{x}_{\mathrm{x}} / \mathrm{GaN} / \mathrm{Sapphire}$}

Table 4.2 shows that the thermal expansion coefficient of AlN is lower than that of the sapphire substrate. Due this difference in the thermal expansion coefficients the AlN films on $\mathrm{SiO}_{x}$ are expected to have a compressive residual stress at room temperature. Infrared transmission measurements could not be extracted for the AlN $(300 \mathrm{~nm}) / \mathrm{SiO}_{\mathrm{x}}(130 \mathrm{~nm}) / \mathrm{GaN}(2 \mu \mathrm{m}) / \mathrm{Sapphire}(450 \mu \mathrm{m})$ heterostructure. This is because of the high reflectivity of the sapphire substrate in the mid-infrared region which prevents the infrared wavelengths from reaching the $\mathrm{AlN}$ film on $\mathrm{SiO}_{\mathrm{x}}$. In addition the information regarding $\mathrm{AlN}$ films on $\mathrm{SiO}_{\mathrm{x}}$ could not be obtained in the reflection mode as well, this is due to the overlap of the infrared active phonon modes from the underlying $\mathrm{GaN}$ and sapphire layers with the infrared active phonon modes from the AlN films. The overlap of the modes makes it difficult to identify the infrared modes corresponding to the AlN films.

The optical images of the AIN air-bridges fabricated on the GaN/Sapphire substrate show a telephone chord pattern during the removal of the sacrificial layer. This pattern can be related to thin films which are under high compressive stress. These type of films delaminate from the surface in a periodic pattern and relieving in the residual stress in the process as shown in Figure 4.30. Based on the telephone chord pattern observed through the optical microscope it can be concluded that the as-grown AlN films $\mathrm{SiO}_{\mathrm{x}}(130 \mathrm{~nm}) / \mathrm{GaN}(2 \mu \mathrm{m}) /$ Sapphire $(450 \mu \mathrm{m})$ are in a compressive stress at room temperature. Table 4.5 summarizes the residual stress of AlN films for the structures considered in section 4.6.1 and 4.6.2.

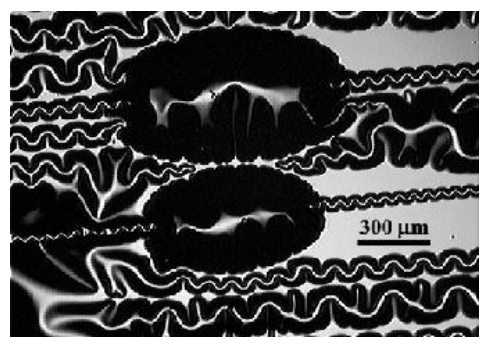

Figure 4.30: Phone chord buckling delamination pattern [98] 
Table 4.5: Residual stress in AlN films on $\mathrm{SiO}_{\mathrm{x}}$ at room temperature

\begin{tabular}{|l|l|}
\hline Multilayers & $\begin{array}{l}\text { Residual stress in AlN } \\
\text { due to thermal mismatch }\end{array}$ \\
\hline $\mathrm{AlN}(300 \mathrm{~nm}) / \mathrm{SiO}_{\mathrm{x}}(130 \mathrm{~nm}) / \mathrm{Si}(350 \mu \mathrm{m})$ & Tensile \\
\hline $\begin{array}{l}\mathrm{AlN}(300 \mathrm{~nm}) / \mathrm{SiO}_{\mathrm{x}}(130 \mathrm{~nm}) / \mathrm{GaN}(2 \mu \mathrm{m}) / \mathrm{Sapphire} \\
(450 \mu \mathrm{m})\end{array}$ & Compressive \\
\hline
\end{tabular}




\section{Chapter 5. Piezoelectric characterization of AIN films on $\mathrm{SiO}_{\mathrm{x}}$}

\subsection{Converse piezoelectric properties of AIN films on $\mathrm{SiO}_{\mathrm{x}}$ :}

The piezoelectric constitutive equations in section 2.2.2 for a wurtzite crystal, describing the electromechanical coupling effect are shown below:

$$
\begin{aligned}
& {\left[\begin{array}{l}
S_{1} \\
S_{2} \\
S_{3} \\
S_{4} \\
S_{5} \\
S_{6}
\end{array}\right]=\left[\begin{array}{cccccc}
s_{11} & s_{12} & s_{13} & 0 & 0 & 0 \\
s_{12} & s_{11} & s_{13} & 0 & 0 & 0 \\
s_{13} & s_{13} & s_{33} & 0 & 0 & 0 \\
0 & 0 & 0 & s_{44} & 0 & 0 \\
0 & 0 & 0 & 0 & s_{44} & 0 \\
0 & 0 & 0 & 0 & 0 & 2\left(s_{11}-s_{12}\right)
\end{array}\right] \cdot\left[\begin{array}{l}
T_{1} \\
T_{2} \\
T_{3} \\
T_{4} \\
T_{5} \\
T_{6}
\end{array}\right]+\left[\begin{array}{ccc}
0 & 0 & d_{31} \\
0 & 0 & d_{31} \\
0 & 0 & d_{33} \\
0 & d_{15} & 0 \\
d_{15} & 0 & 0 \\
0 & 0 & 0
\end{array}\right] \cdot\left[\begin{array}{l}
E_{1} \\
E_{2} \\
E_{3}
\end{array}\right]} \\
& {\left[\begin{array}{l}
D_{1} \\
D_{2} \\
D_{3}
\end{array}\right]=\left[\begin{array}{cccccc}
0 & 0 & 0 & 0 & d_{15} & 0 \\
0 & 0 & 0 & d_{15} & 0 & 0 \\
d_{31} & d_{31} & d_{33} & 0 & 0 & 0
\end{array}\right] \cdot\left[\begin{array}{l}
T_{1} \\
T_{2} \\
T_{3} \\
T_{4} \\
T_{5} \\
T_{6}
\end{array}\right]+\left[\begin{array}{ccc}
\varepsilon_{11} & 0 & 0 \\
0 & \varepsilon_{11} & 0 \\
0 & 0 & \varepsilon_{33}
\end{array}\right] \cdot\left[\begin{array}{l}
E_{1} \\
E_{2} \\
E_{3}
\end{array}\right]}
\end{aligned}
$$

Considering the converse piezoelectric effect, the piezoelectric coefficients that are active are the $d_{33}, d_{31}$ and $d_{15}$ components. In the converse piezoelectric effect the strain ' $S$ ' generated in a piezoelectric material, in the absence of an external mechanical stress, depends on the direction along which the electric field is applied. In a wurtzite crystal the external electric field is typically applied either parallel to or perpendicular to the caxis. When an electric field is applied along the c-axis it is called the longitudinal field excitation and when an electric field is applied perpendicular to the c-axis it is called the lateral field excitation.

\subsubsection{Measurement of longitudinal piezoelectric coefficient of AIN films by laser Doppler vibrometer:}

When an external electric field is applied along the c-axis i.e $E_{3}$, in the absence of any external stress a strain is generated in the crystal purely due to the electric field. 
Shown in Figure 5.1 is the schematic of a wurtzite crystal with an electric field applied along the c-axis along with the modified constitutive equations:

(b)

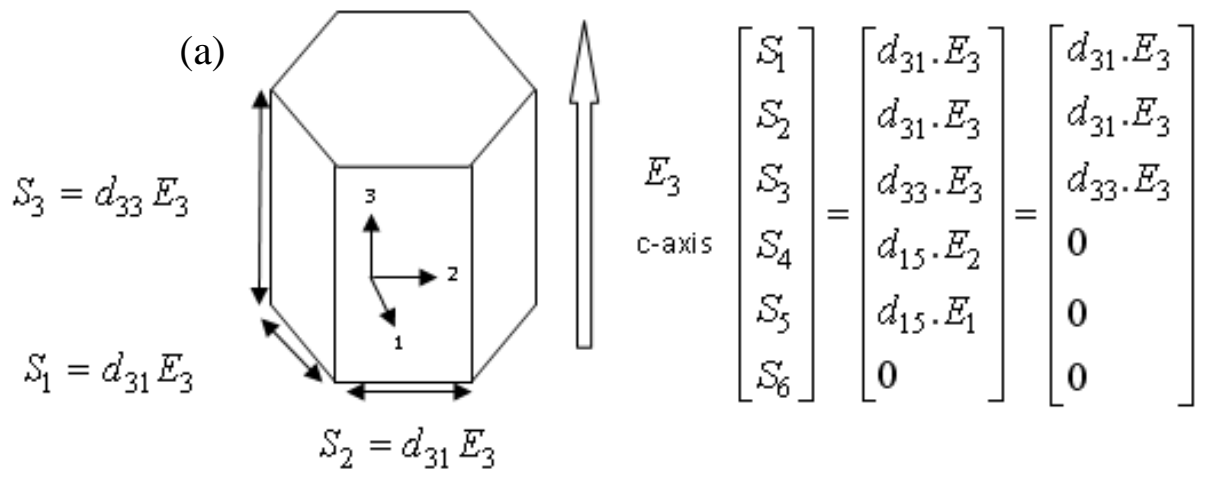

Figure 5.1: (a) Schematic of wurtzite crystal with electric field applied along the c-axis (b) Modified constitutive equations [100]

The equations describe that the electric field ' $E_{3}$ ' couples only with the $d_{33}$ and $d_{31}$ components thereby generating a strain along the c-axis and in the basal plane respectively. The AlN films on Si (111) substrate, grown by the MOVPE techniques are preferentially oriented along the c-axis. When an external electric field is applied across the film it results in a strain along the c-axis. The strain along the c-axis leads to a change in the thickness of the film. Typical values of $\mathrm{d}_{33}$ for AlN films are between 3.9 $\mathrm{pm} / \mathrm{V}-7 \mathrm{pm} / \mathrm{V}$ [1]. The change in the thickness of the film is usually in picometers for a voltage with an amplitude of tens of volts. This change in thickness value can be detected by a laser Doppler vibrometer.

In the laser Doppler vibrometry set up a laser beam is focused onto a vibrating sample. The vibrating sample induces a Doppler shift in the frequency of the incident laser beam. Based on the frequency shift of the reflected beam, the amplitude of the vibration and frequency of vibration of the sample can be extracted. Figure 5.2 shows the schematic of the setup for measuring the thickness change of a piezoelectric film grown on a conductive substrate. 

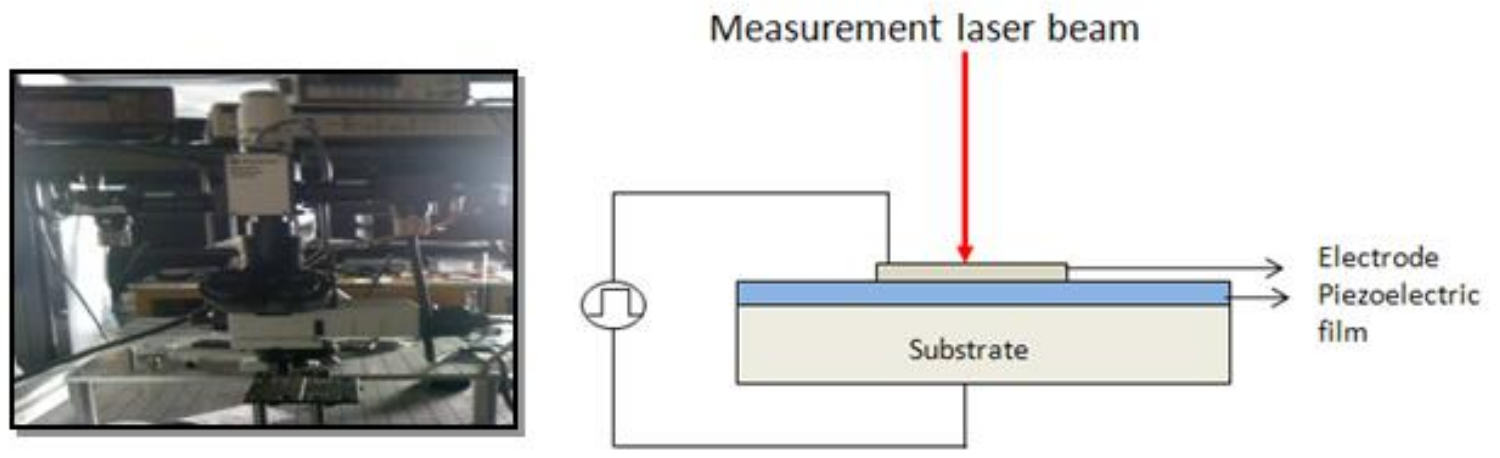

Figure 5.2: Measurement of the thickness change of a piezoelectric film by laser Doppler vibrometer

On applying an AC field across the thickness of the film the strain in the film changes periodically. The thickness of the film also changes periodically in response to periodic variation of the strain. The piezoelectric film is set into oscillations by applying an AC electric field across the thickness the substrate and the top electrode. The piezoelectric coefficient $d_{33}$ is given by:

$$
d_{33}=\frac{\text { Amplitude of vibration }}{\mathrm{V}_{\mathrm{ac}}}
$$

Where $\mathrm{V}_{\mathrm{ac}}$-Amplitude of the AC voltage

When the thickness of the film changes in response to the external electric field, the top electrode also moves by an equal distance in the vertical direction and Doppler shifts the incident laser beam. The $\mathrm{d}_{33}$ measurements were taken on a square platinum top electrode with an area of $100 \mu \mathrm{m} \times 100 \mu \mathrm{m}$. The measurements were taken on the AlN films grown on silicon substrate i,e in between the $\mathrm{SiO}_{x}$ stripes as shown in Figure 5.3. A platinum film of thickness $100 \mathrm{~nm}$ was sputtered on the bottom of the silicon substrate, which serves as the bottom electrode. An AC voltage was applied across the top and the bottom electrode 
(a)

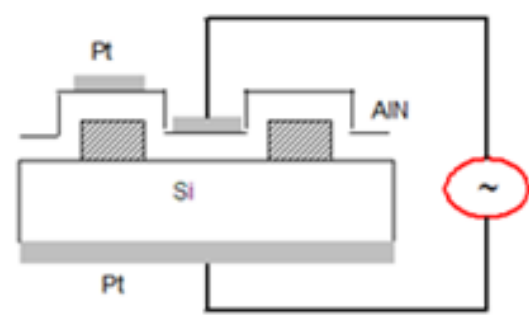

(b)

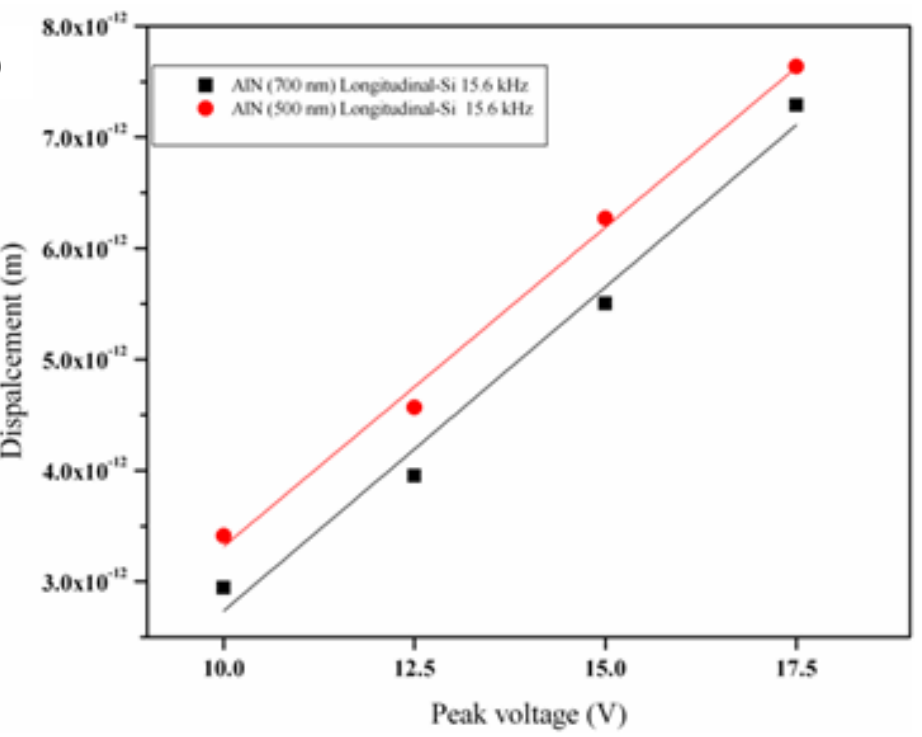

Figure 5.3: (a) Piezoelectric measurement of the AlN films between $\mathrm{SiO}_{x}$ stripes with platinum back and top contacts (b) Apparent piezoelectric coefficient of AlN films grown on silicon substrate.

Figure 5.3 (b) shows the measured amplitude of vibration or maximum displacement of the top electrode. The amplitude of vibration of the top electrode increases linearly with an increase in the amplitude of the AC voltage. The constitutive equations predict that the change in the thickness of the piezoelectric film increases linearly with an increase in the amplitude of the electric field. Therefore the top electrode maximum displacement also increases with increasing amplitude of the AC voltage. The $\mathrm{d}_{33}$ value can be estimated from the slope of the displacement vs voltage plot.

The apparent $d_{33}$ values for AIN films of different thicknesses as calculated from the slope of the plots in Figure 5.3 (b) are shown in Table 5.1

Table 5.1: Apparent $d_{33}$ value of the AlN film grown on silicon substrate

\begin{tabular}{|c|c|}
\hline & Slope $(\mathrm{m} / \mathrm{V})$ \\
\hline AlN $(700 \mathrm{~nm})$ & $0.574 * 10^{-12}$ \\
\hline AlN $(500 \mathrm{~nm})$ & $0.58 * 10^{-12}$ \\
\hline
\end{tabular}


Table 5.1 shows that the apparent $d_{33}$ values are much lower than the theoretical value of $3.98 \mathrm{pm} / \mathrm{V}$. The AlN film is clamped in-plane to the silicon substrate due to this expansion and contraction of the film is constrained. It has been determined that the for an in-plane clamped film the measured piezoelectric coefficient reduced and is given by:

$$
d_{33}^{f}=d_{33}-\frac{2 d_{31} c_{13}^{f}}{\mathbf{C}_{11}^{f}+c_{12}^{f}}-[101]
$$

Where, $d_{33}$ and $d_{31}$ are the piezoelectric coefficients of the free standing film. The second term leads to a reduced $\mathrm{d}_{33}$ measured value. And are the elastic compliances of the film.

However the electric field ' $\mathrm{E}_{3}$ ' field also contributes to the expansion of the basal plane by coupling through the $\mathrm{d}_{31}$ piezoelectric coefficient. As the AlN film is laterally clamped to the silicon substrate, the thin film and substrate together behave as a piezoelectric bimorph. Therefore the substrate bends as the AIN film expands in the basal plane as shown in Figure 5.4. The bending deflection is approximately given by [101]:

$$
b=\frac{3 h d_{31} E \gamma}{4}\left(\frac{L}{H}\right)^{2}
$$

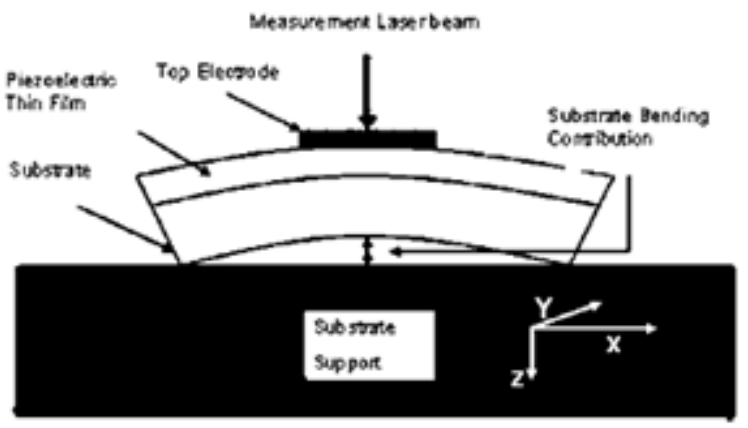

Figure 5.4: Typical thin film substrate configuration showing the influence of substrate on $\mathrm{d}_{33}$ measurement [102].

Where L: Length of the electrode 
H: Thickness of the substrate

$\mathrm{h}$ : Thickness of the piezoelectric film

E: Youngs modulus of the substrate

The bending of the substrate was minimized by attaching the silicon substrate to an aluminum metal block and choosing an electrode with a smaller length. However, the total displacement of the top electrode consists of contributions from thickness change of the film and deflection due to the substrate bending effect. Therefore the observed displacement does not give a true measure of the $\mathrm{d}_{33}$ piezoelectric coefficient.

$$
d_{33}^{f}=d_{33}-\frac{2 d_{31} c_{13}^{f}}{\mathbf{৫}_{11}^{f}+c_{12}^{f}-}+\frac{2 d_{31} c_{13}^{s} \gamma}{\boldsymbol{\bigotimes}_{11}^{s}+c_{12}^{s}}-[101]
$$

Where, the last term on the right represents the positive contribution from the substrate.

Taking into account the above factors it can be concluded that the measurement of the longitudinal piezoelectric strain coefficient by laser Doppler vibrometer gives only the apparent value. But this technique can be used as check to investigate the piezoelectric properties of thin films.

\subsubsection{Measurement of longitudinal piezoelectric coefficient of AIN films by laser Doppler vibrometer:}

For the case of an external electric field applied perpendicular to the c-axis the constitutive equations reduce to the following form as shown in Figure 5.5. The reduced constitutive equations show that the external electric field couples only to the $d_{15}$ coefficient generating a shear strain. 
(a)

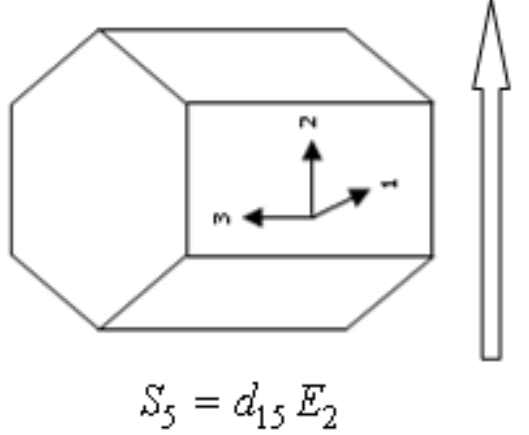

(b) $\left[\begin{array}{l}S_{1} \\ S_{2} \\ S_{3} \\ S_{4} \\ S_{5} \\ S_{6}\end{array}\right]=\left[\begin{array}{l}d_{31} \cdot E_{3} \\ d_{31} \cdot E_{3} \\ d_{33} \cdot E_{3} \\ d_{15} \cdot E_{2} \\ d_{15} \cdot E_{1} \\ 0\end{array}\right]=\left[\begin{array}{l}0 \\ 0 \\ 0 \\ d_{15} E_{2} \\ 0 \\ 0\end{array}\right]$

Figure 5.5: (a) Schematic of wurtzite crystal with electric field applied normal to the caxis (b) Modified constitutive equations [100].

The shear strain consists of two components as shown in Figure 5.6.

(a)

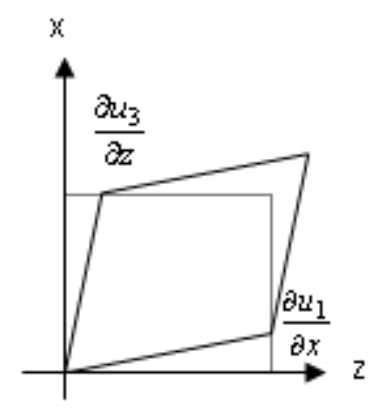

Figure 5.6: Components in a shear strain [102]

$$
\varepsilon_{x z}=\frac{1}{2}\left(\frac{\partial u_{1}}{\partial x}+\frac{\partial u_{3}}{\partial z}\right)
$$

The shear components $\frac{1}{2}\left(\frac{\partial u_{1}}{\partial x}\right)$ and $\frac{1}{2}\left(\frac{\partial u_{3}}{\partial z}\right)$ basically describe the deformation along the $\mathrm{x}$ and $\mathrm{z}$ directions. In case of a piezoelectric film depending on the dimensions of the film and the electrode configuration one of the two shear strains dominates as shown in Figure 5.7. The shaded areas are the electrodes and the c-axis is indicated by an arrow and labeled as P. 

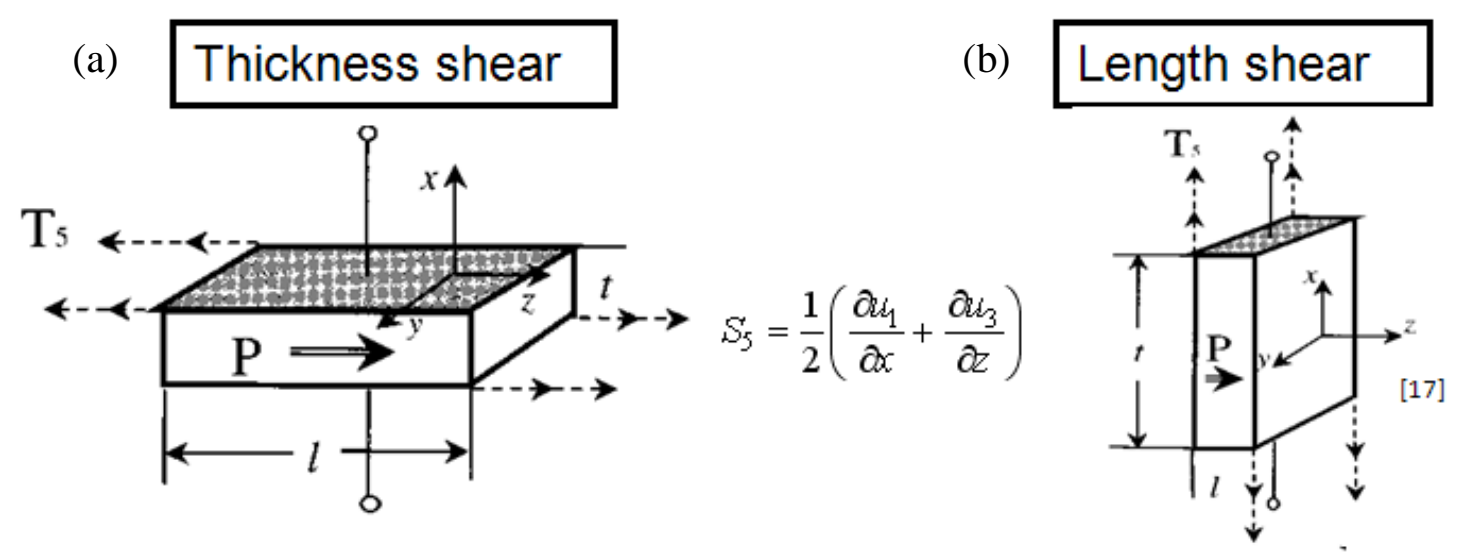

Figure 5.7: (a) Thickness shear strain (c) Length shear strain [103]

Accordingly the shear strain components are now classified as thickness shear strain and the length shear strain. When the spacing between the electrodes is less than the thickness of the film i.e $t<l$ as shown in Figure 5.7 (a) the term $\frac{\partial u_{1}}{\partial x}$ dominates the strain equation and the deformation of the film is primarily along the polarization axis or the caxis. When the spacing between the electrodes is greater than the thickness of the piezoelectric film i.e $t>l$ the term $\frac{\partial u_{3}}{\partial z}$ dominates the shear strain equation and the deformation is primarily along the axis perpendicular to the c- axis.

In a bulk piezoelectric crystal electrodes can be placed parallel to the c-axis as shown in Figure 5.7. This electrode configuration gives rise to an external electric field perpendicular to the c-axis. But in thin films due to their finite thickness the preferred method to generate an electric field perpendicular to the c-axis is to deposit a pair of electrodes on the surface of the film. This method of generating a shear strain is called the lateral field excitation. As shown in Figure 5.8, when the spacing between two electrodes increases the electric field in the piezoelectric film in-between the electrodes is essentially perpendicular to the c-axis . 

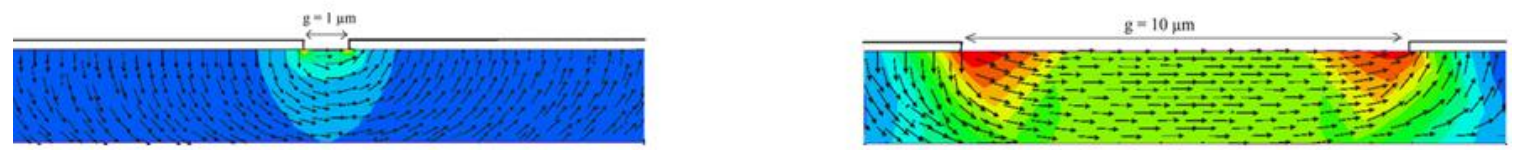

Figure 5.8: Electric field generated in a piezoelectric film with electrode gaps (a) $1 \mu \mathrm{m}$ (b) $10 \mu \mathrm{m}$. Modified from [104].

\section{Shear strain in AIN films grown on silicon}

In order to excite shear strain in the AIN film a pair of platinum electrodes separated by a gap of $70 \mu \mathrm{m}$ were deposited on the AlN films grown on silicon substrate as shown in Figure 5.9. The AlN films on crystalline silicon substrate exhibit a preferentially caxis orientation. In a c-axis oriented AIN thin film the polarization axis is perpendicular to the surface of the film. Given the gap of $70 \mu \mathrm{m}$ between the electrodes the pair of electrodes the electric field in the film is expected to be perpendicular to the c-axis in between the electrodes.
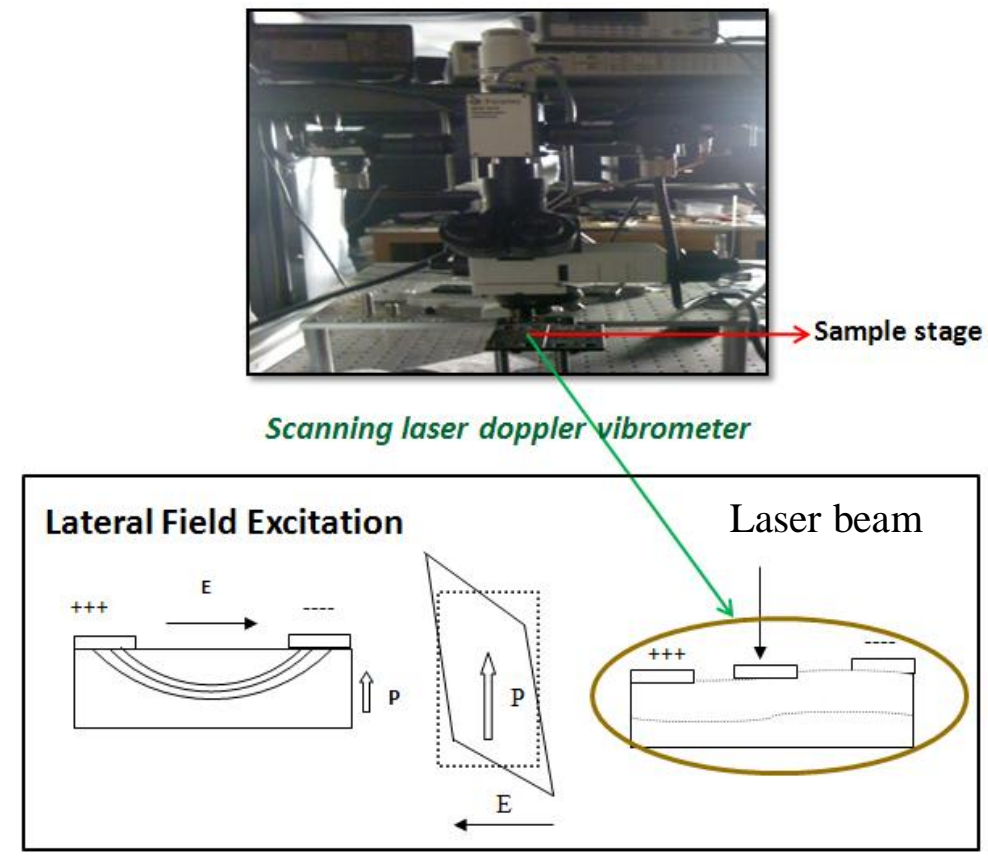

Figure 5.9: Activation of thickness shear strain in AlN films through lateral field excitation 
Under these conditions shear strains can be excited in the AlN films. As the spacing between the electrodes is much larger than the thickness of the AlN film, the length shear strain mode is expected to dominate. On applying an AC electric field across the platinum electrodes, periodic thickness shear and length shear modes are generated between the electrodes. The periodic displacement normal to the surface of the film was probed by laser Doppler vibrometry, by reflecting the laser of a metal contact deposited between the electrodes as shown in Figure 5.9.

AC voltages with different frequencies and amplitudes were applied across the platinum electrodes through a function generator. The plots in Figure 5.10 show an increase in the displacement of the top electrode with an increase in the applied electric field as predicted by the constitutive equations. However, as the thin film is clamped inplane to the silicon substrate the displacement detected by the vibrometer does not give the true shear strain.

Lateral Field Excitation
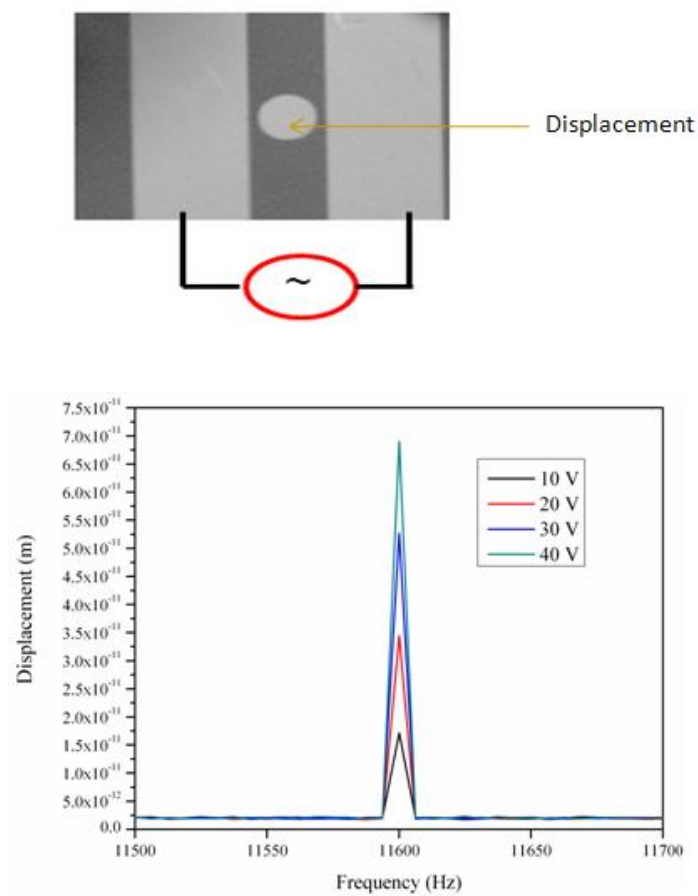
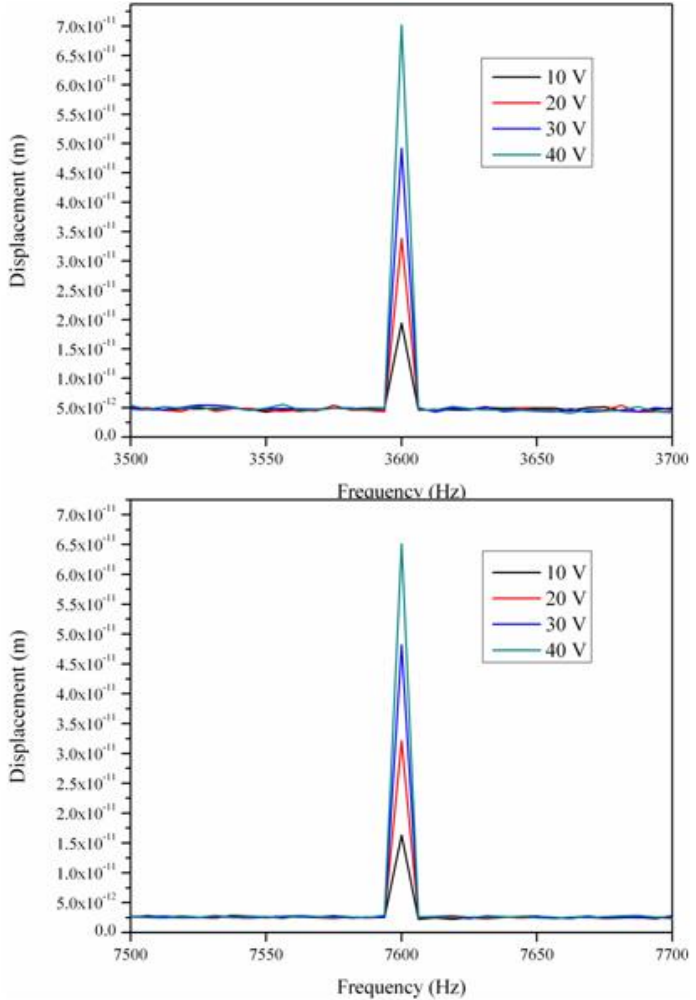

Figure 5.10: Piezoelectric response of AlN films actuated by lateral field excitation 
Figure 5.11 shows that the maximum displacement of the top electrode increases with an increase in the amplitude of the AC voltage.

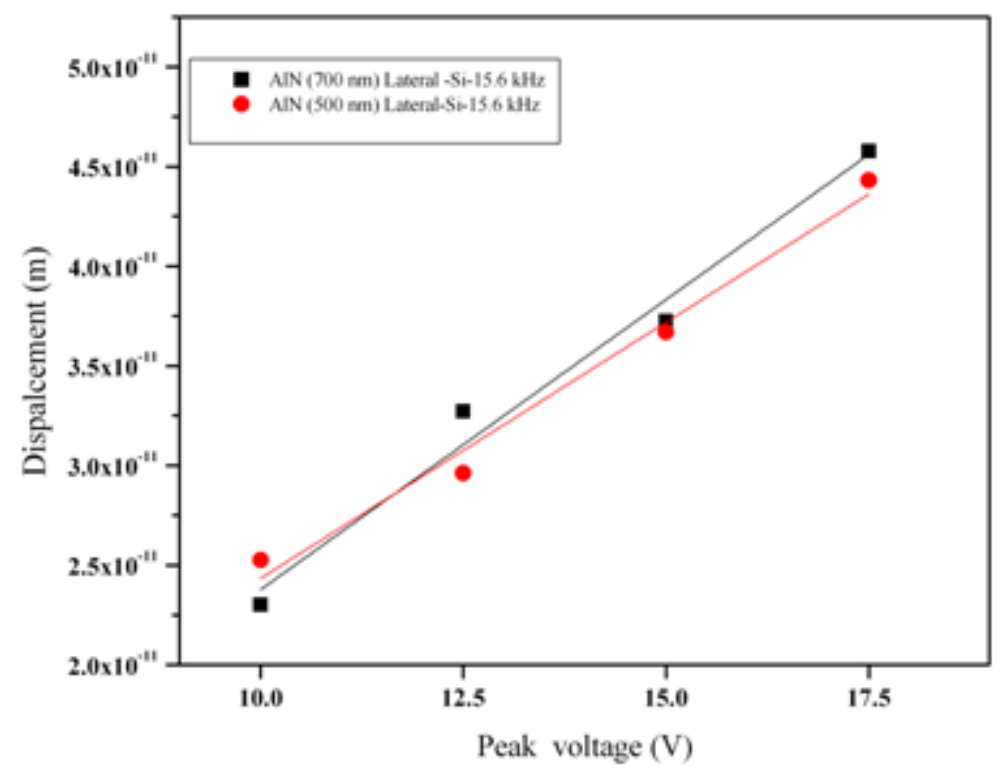

Figure 5.11: Displacement of top electrode for a laterally actuated AIN films grown on silicon.

Table 5.2 shows the slope extracted by linear fitting. A comparison of the slope values for laterally actuated and longitudinally actuated in-plane clamped AlN films shows that the displacement obtained by lateral field excitation is higher when compared to that of a longitudinally actuated film. Based on the above analysis it can be concluded that similar to the longitudinal field excitation techniques lateral field excitation can also be used identify piezoelectric films.

Table 5.2: Slope of displacement of top electrode actuated by lateral field techniques

\begin{tabular}{|c|c|}
\hline & Slope $(\mathrm{m} / \mathrm{V})$ \\
\hline AlN $(700 \mathrm{~nm})$ & $2.91084 * 10^{-12}$ \\
\hline AlN $(500 \mathrm{~nm})$ & $2.56952 * 10^{-12}$ \\
\hline
\end{tabular}




\subsubsection{Piezoelectric response of $\mathrm{AIN}$ films on $\mathrm{SiO}_{\mathrm{x}}$ measured by laser Doppler vibrometer:}

In order to test the piezoelectric response of the $\mathrm{AlN}$ films on $\mathrm{SiO}_{\mathrm{x}}$ the films were actuated in the longitudinal and lateral field excitation modes as shown in Figure 5.12
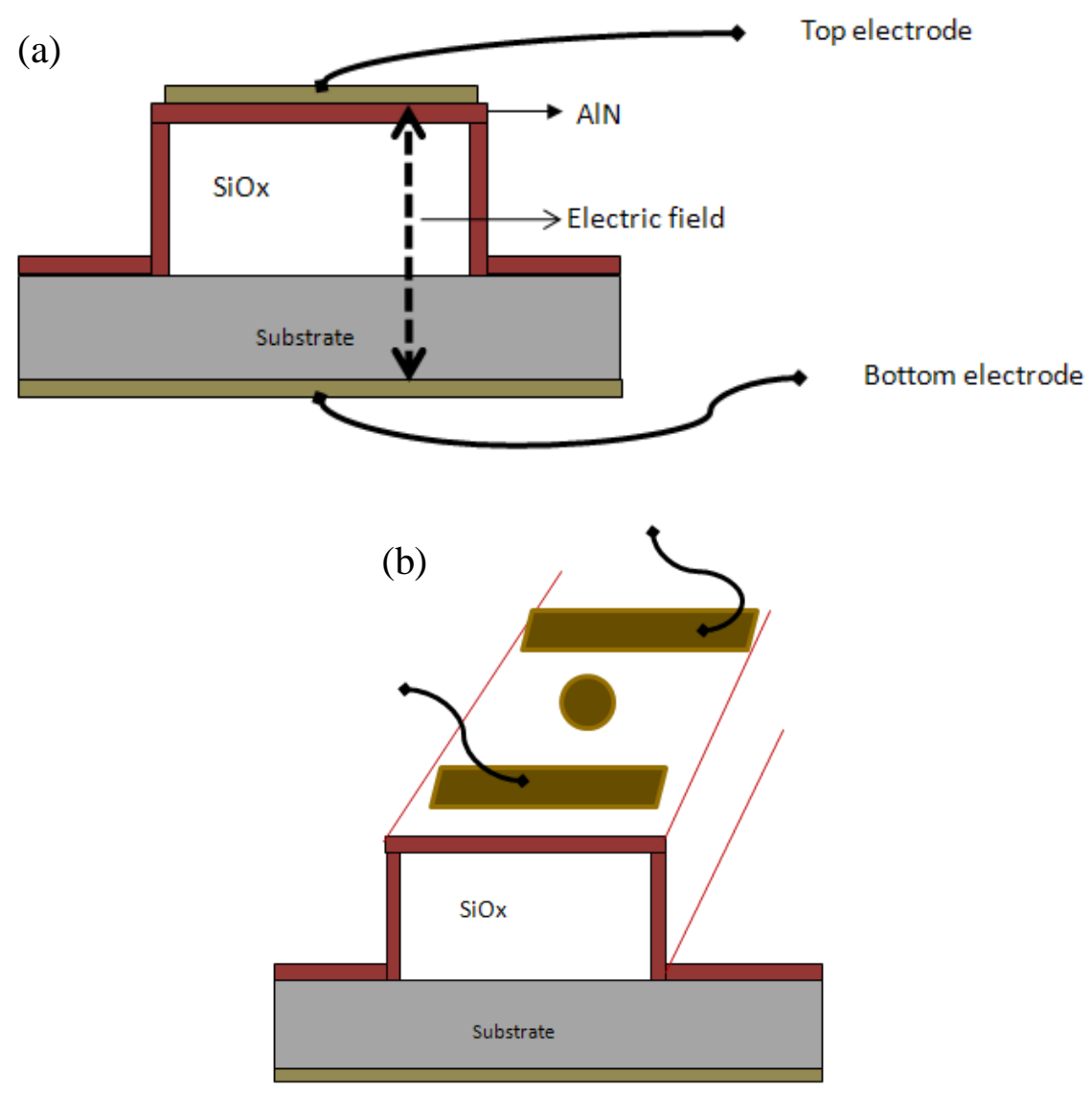

Figure 5.12: (a) Longitudinal field actuation (b) Lateral field actuation of $\mathrm{AlN} / \mathrm{SiO}_{\mathrm{x}}$ with a platinum back and top electrode.

In the longitudinal field excitation mode the applied external electric field is distributed across the layers between the top and bottom platinum electrodes. Pt back electrode forms an ohmic contact with the highly doped p-Si. Due to the high conductivity of the silicon substrate much of the voltage is distributed across the $\mathrm{AlN} / \mathrm{SiO}_{\mathrm{x}}$ bilayer. Ignoring the fringe field effects the voltage distribution across the $\mathrm{AlN} / \mathrm{SiO}_{\mathrm{x}}$ is given by: 


\author{
Where $\quad$ Voltage across the AlN film on $\mathrm{SiO}_{\mathrm{x}}$ \\ : Voltage across the $\mathrm{SiO}_{\mathrm{x}}$ film \\ : Thickness of the AlN film \\ : Thickness of the $\mathrm{SiO}_{\mathrm{x}}$ film
}

Eq 5.6 shows that the as the thickness of AlN film on $\mathrm{SiO}_{\mathrm{x}}$ increases the voltage drop across the AlN film increases. As a result, with an increase in the thickness of the AlN film the displacement of the top electrode should increase for a constant electric field. But the longitudinal excitation of AlN films with thicknesses of $300 \mathrm{~nm}, 500 \mathrm{~nm}$ and 700 nm did not yield any measureable displacements during the vibrometer measurements. Prior work [83] and the discussion in section 4.5 shows that the AlN films exhibit a preferentially c-axis orientation but have a lower crystal quality.

The piezoelectric response of the as-grown AlN films on $\mathrm{SiO}_{\mathrm{x}}$ was tested by the lateral field excitation techniques. In order to excite shear strain in the film a pair of platinum electrodes separated by a gap of $70 \mu \mathrm{m}$ were deposited on the AlN films grown on $\mathrm{SiO}_{\mathrm{x}}$ as shown in Figure 5.13 (b). The AlN films on $\mathrm{SiO}_{\mathrm{x}}$ were actuated by applying an AC electric field across the platinum electrodes. The laser Doppler vibrometer measurements show a displacement at the driving frequency of the applied voltage. This shows that the AlN films on $\mathrm{SiO}_{\mathrm{x}}$ exhibit piezoelectric properties. Figure 5.13 shows a comparison of plot of the top electrode displacement versus applied AC voltage for AIN films grown on silicon substrate and $\mathrm{SiO}_{\mathrm{x}}$. 

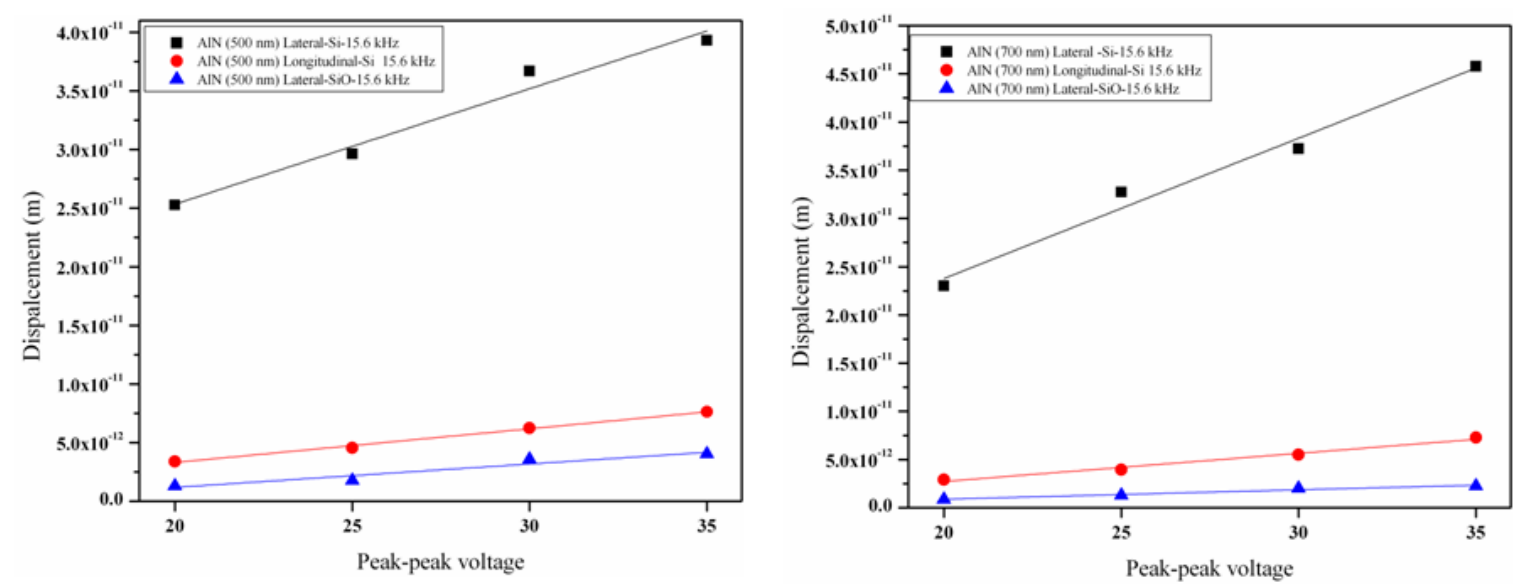

Figure 5.13: Comparison of piezoelectric response of AlN films grown on Silicon substrate and $\mathrm{SiO}_{\mathrm{x}}$ sacrificial layer

Figure 5.13 shows that the displacement of the AlN films grown on $\mathrm{SiO}_{x}$, obtained by lateral field excitation techniques, have a significantly lower displacement values when compared to the AlN films grown on silicon substrate. Figure 5.14 also shows that the displacements of $\mathrm{AlN}$ films on $\mathrm{SiO}_{\mathrm{x}}$ excited by lateral field are lower than the displacements of the AIN films on silicon excited by longitudinal excitation. This behavior can be attributed to the lower crystal quality of the AIN films on $\mathrm{SiO}_{\mathrm{x}}$. FTIR measurements show the AlN film structure consists of non c-axis oriented grains which indicates the presence grains of opposite polarity. Grains of opposite polarity are responsible for the reduced net piezoelectric response observed in $\mathrm{AlN}$ films on $\mathrm{SiO}_{\mathrm{x}}$ [105], [106].

\subsubsection{Piezoelectric actuation of AIN air-bridges}

For actuating AlN air-bridges two methods were adopted as shown in Figure 5.14. Figure 5.14(a) shows the piezoelectric actuation of the AlN air-bridge by applying electric field across the air-gap and the AIN film. The voltage distribution follows Eq 5.6 with the thickness of $\mathrm{SiO}_{\mathrm{x}}$ replaced by air-gap with a dielectric constant equal to 1 . This configuration results in the actuation of the air-bridge though a combination of piezoelectric action from the AIN film and electrostatic actuation due to the air-gap. The movement of the air-bridge results in a pumping action. 
(a)
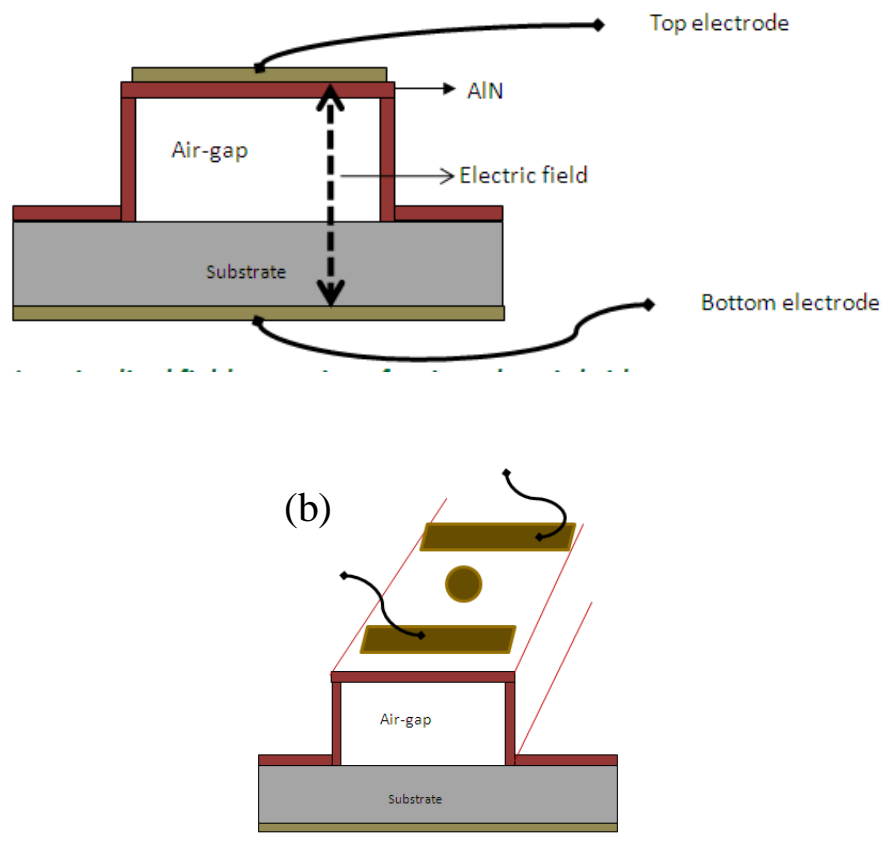

Figure 5.14: (a) Longitudinal field actuation (b) Lateral field actuation of AlN airbridge with a platinum back and top electrode.

Alternately the air-bridge can be actuated purely by piezoelectric means as shown in Figure 5.14 (b). This technique utilizes the lateral field actuation of AlN air-bridges. Shear strain can be generated in the AlN air-bridges by using a pair of electrodes on the air-bridges. The shear strain generated due to lateral field excitation can be applied to actuate a section of the piezoelectric film to as shown in Figure 5.15

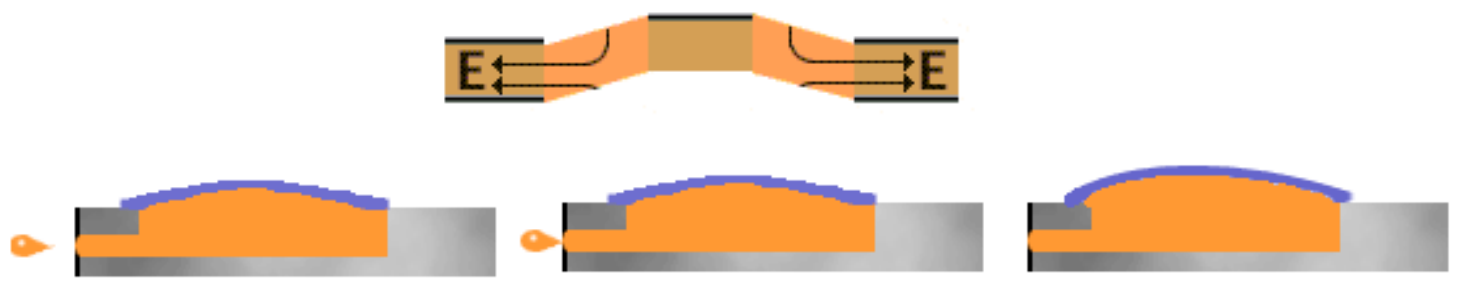

Figure 5.15: Shear mode actuated piezoelectric membrane [107]

This shear deformation leads to a pumping mechanism. The pumping mechanism can be used to move fluids through the channels as shown in Figure 5.15. 


\subsubsection{Fabrication of AIN air-bridges with electrodes}

AlN films on $\mathrm{SiO}_{x}$ of thicknesses $300 \mathrm{~nm}, 500 \mathrm{~nm}$ and $700 \mathrm{~nm}$ were etched in buffered HF solution. The thickness of $\mathrm{SiO}_{\mathrm{x}}$ was around $130 \mathrm{~nm}$. The as-grown samples with electrodes were etched in buffered HF solution to fabricate the AIN air-bridges. Due to the isotropic nature of the wet etching process and the corrosive nature of the AlN film on $\mathrm{SiO}_{\mathrm{x}}$, the sacrificial layer under the $\mathrm{AlN}$ film covered by the electrodes is expected to be etched as well. Shown in Figure 5.16 is the optical image of the AlN airbridge after etching in buffered HF solution. The image shows a delamination of the top electrode. Discussion in section 4.5 shows that the sacrificial layer etching mechanism is enhanced as the material properties of the AIN film changes with wet etching. The etching mechanism attacks the interface between the electrode and the AlN film. In this process the electrodes are released from the surface of the AlN films. Delamination of the electrodes was observed for AlN films of thicknesses $300 \mathrm{~nm}, 500 \mathrm{~nm}$ and $700 \mathrm{~nm}$.

(a)

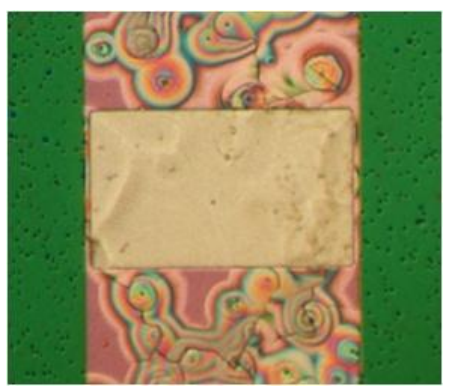

(b)

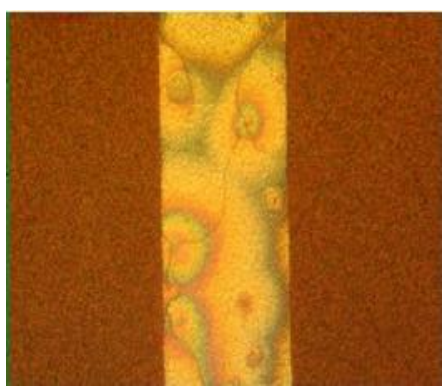

Figure 5.16: Optical images of AlN films (a) $500 \mathrm{~nm}$ (b) $700 \mathrm{~nm}$ grown on $\mathrm{SiO}_{\mathrm{x}}$ after etching for 6.5 hours

However after the removal of the sacrificial layer, the AlN film of thickness $300 \mathrm{~nm}$ exhibited excessive fractures due to a very high tensile stress. The high tensile stress also resulted in the delaminationt of the air-bridges fro the substrate making it difficult to fabricate air-bridges. 
AlN air-bridges of thicknesses $500 \mathrm{~nm}$ and $700 \mathrm{~nm}$ exhibited fractures on the surface as shown in Figure 5.16 (a) and (b) respectively. But the air-bridges were not delaminated from the substrate. This shows that thicker AIN films have a lower residual stress than the AlN films of thickness $300 \mathrm{~nm}$.

The variation in the color of the AlN films is due to the interference pattern generated due to the existence of an air-gap between the AlN film and the substrate. The non uniform etching observed in the optical images as discussed in section 4.5 shows that the quality of the AlN films on $\mathrm{SiO}_{\mathrm{x}}$ is not homogeneous and this results in a non uniform etching pattern on the surface of the AIN films. Figure 5.17 shows the displacement of the delaminated area of the top electrode of the AlN air-bridges of $500 \mathrm{~nm}$ thick actuated through longitudinal excitation. The displacement of the top electrode was measured on the delaminated area.
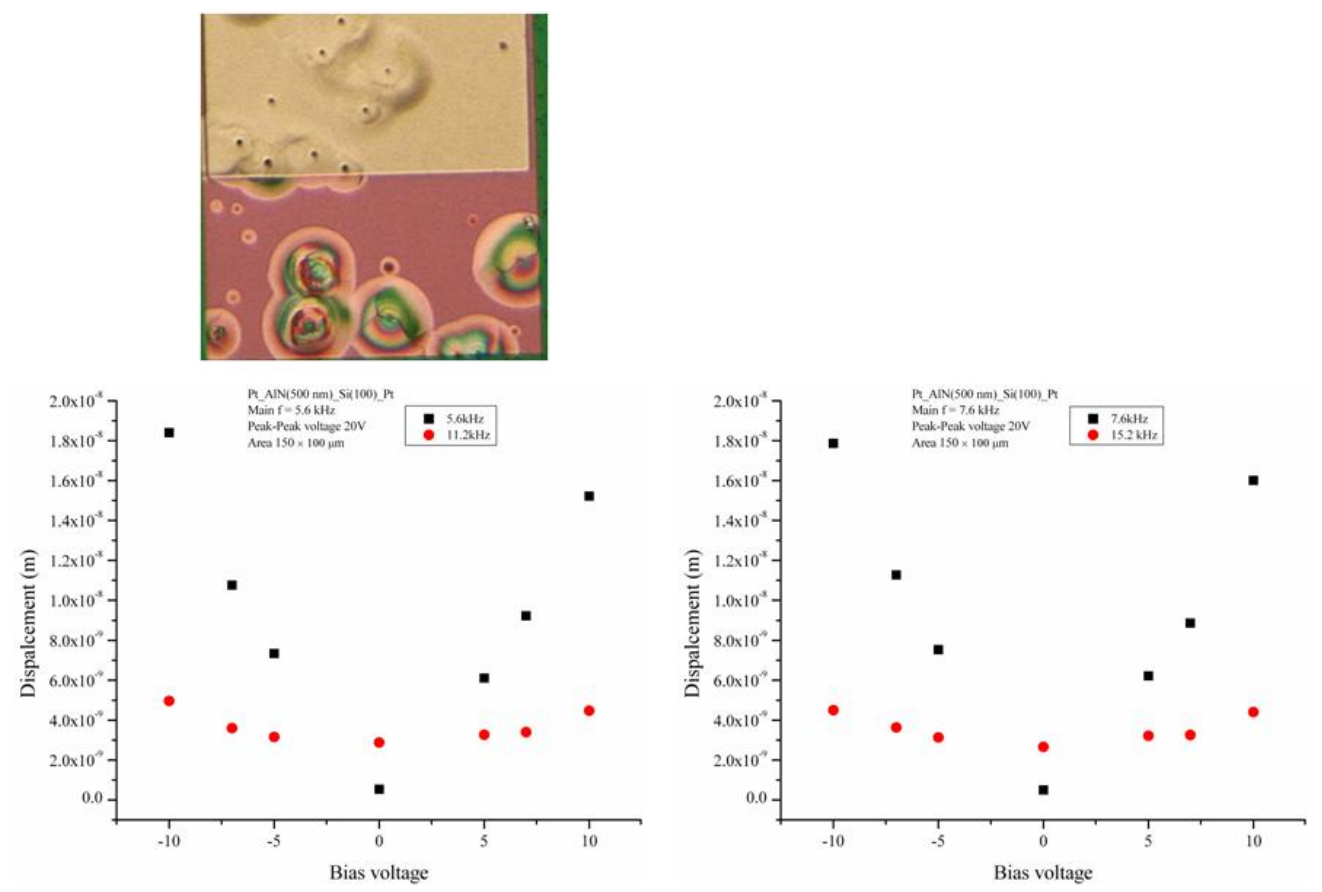

Figure 5.17: Displacement of the AlN air-bridges observed of delaminated contacts after actuated by longitudinal field excitation. 
The measured maximum displacement varies with the bias voltage. This indicates that the actuation mechanism is a combination of both the electrostatic and piezoelectric actuation. However, the contribution of the delaminated section of the electrode to the measured displacement is unclear at this point.

Platinum contacts were re-sputtered on the etched air-bridges. However due to the increased surface roughness of the AlN films with thickness $700 \mathrm{~nm}$ after the wet etching process, the platinum contacts did not have adhesion to the surface of the air-bridges. Platinum electrodes could only be sputtered on AlN air-bridges with thickness of $500 \mathrm{~nm}$. Figure 5.18 shows the optical images of the bridges after sputtering platinum electrode. The thickness of the platinum electrodes sputtered for as-grown AIN films and AIN airbridges was around $100 \mathrm{~nm}$.

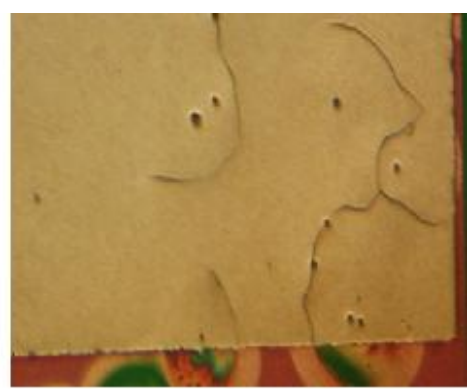

Figure 5.18: Optical images of the AlN air-bridge after re-sputtering platinum contacts on the bridges 

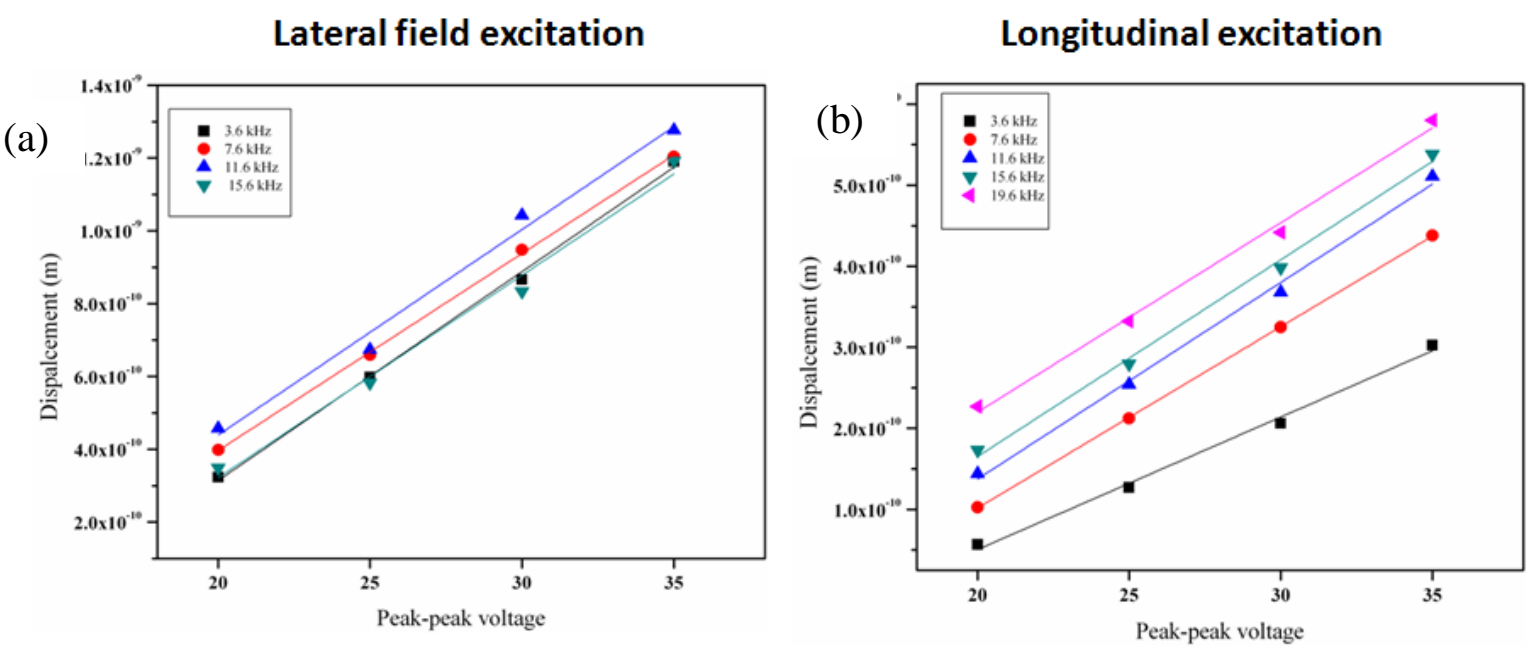

Figure 5.19: Displacement of the AlN air-bridge in the (a) lateral field actuation (b) Longitudinal field actuation configuration.

The optical images show visible cracks even after re-sputtering $100 \mathrm{~nm}$ platinum. Figure 5.19 shows the displacement of the re-sputtered electrode as function of the applied AC voltage in the lateral field and longitudinal field actuation configuration. The top electrode shows significant displacements upto $1 \mathrm{~nm}$. The increase in the displacement is due to etching of the sacrificial layer which removes the in-plane clamping, thereby allowing free movement of the AlN film. But the presence of the cracks complicates the understanding of the piezoelectric properties of the AlN airbridges. Figure 5.20 shows the slope obtained from the plot in Figure 5.19. A higher slope is an indication of an increase in the displacement with an increase in the amplitude of the voltage. While the slope obtained from lateral field excitation is essentially constant for different frequencies the slopes obtained from the longitudinal actuation shows a gradual increase and decrease with an increase in the frequency. This shows that the longitudinal excitation generates a resonance effect. The resonance effect is a combination both the piezoelectric and electrostatic effect. 
(a)

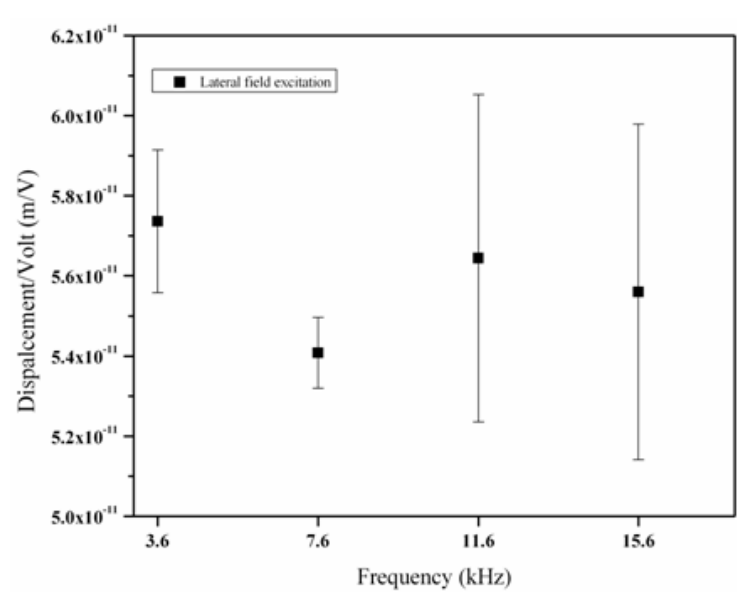

(b) Longitudinal field excitation

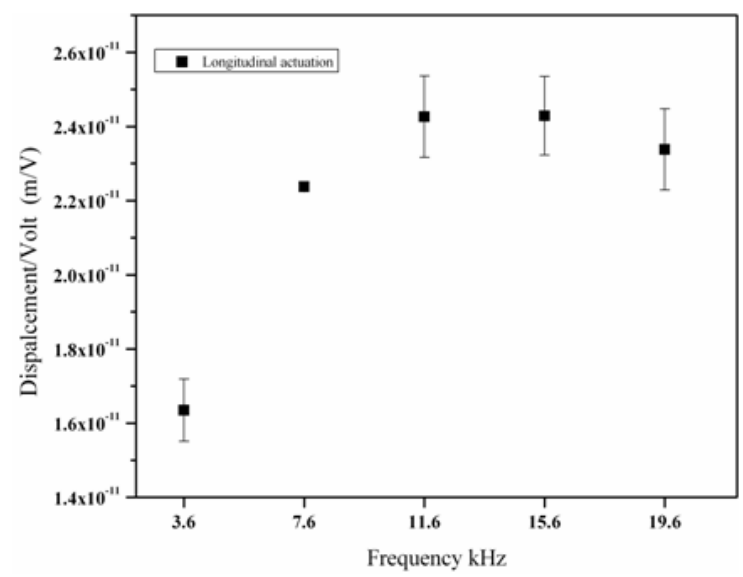

Figure 5.20: Slope of the (a) Lateral field actuated AlN air-bridges (b) Longitudinal field actuated AIN air-bridges. 


\section{Chapter 6. Conclusions and future work}

AlN air-bridges have been fabricated using silicon oxide sacrificial layers by metal organic techniques. Infrared transmissions techniques were developed to determine the crystallographic orientation of AlN thin films. The measurements show that the asgrown AlN films on $\mathrm{SiO}_{x}$ are in a predominantly wurtzite phase. Micro-Raman techniques show that AlN air-bridges are preferentially c-axis oriented. Lateral field excitation techniques were developed to determine the piezoelectric response of AlN films grown on $\mathrm{SiO}_{\mathrm{x}}$. The results show that the AlN films grown on $\mathrm{SiO}_{\mathrm{x}}$ exhibit weak piezoelectric properties. The high growth temperature resulted in significant residual stress leading to cracking of the AlN air-bridges grown on Silicon substrate.

Future work need to address the issues of residual stress in order to fabricate suspended piezoelectric AlN air-channels for micro nanofluidic channel applications. Alternate substrates such as sapphire can be used to fabricate the air-bridges. But the piezoelectric response, and device geometry, of the AlN air-bridges fabricated on sapphire substrates needs to be investigated to prove its usefulness. 


\section{Bibliography}

[1] V. Cimalla, J. Pezoldt, O. Ambacher, "Group III nitride and SiC based MEMS and NEMS: materials properties, technology and applications," J. Phys. D: Appl. Phys. , vol. 40, no. 20, pp. 6386-434, Oct. 2007.

[2] R.C. Turner, P.A. Fuierer, R.E. Newnham, T.R. Shrout, "Materials for high temperature acoustic and vibration sensors: A review," Appl. Acoust. , vol. 41, no. 4, pp. 299-324, Feb. 1994.

[3] O. Ambacher, M. Eickhoff, A. Link, M. Hermann, M. Stutzmann, F. Bernardini, V. Fiorentini, Y. Smorchkova, J. Speck, U. Mishra, W. Schaff, V. Tilak, L. F. Eastman, "Electronics and sensors based on pyroelectric AlGaN/GaN heterostructures," Phys. Status Solidi C, vol. 0, no. 6, pp. 1878-1907, Aug. 2003.

[4] K. Tsubouchi, K. Sugai, N. Mikoshiba, "AlN material constants evaluation and SAW properties on AlN/Al2O3and AlN/Si," in IEEE Ultrasonic Symp. Proc., San Diego, 1981, pp. 375-380.

[5] I.L. Guy S. Muensit, E.M. Goldys , Appl. Phys. Lett., vol. 75 , p. 4133, 1999.

[6] S. Muensit, E.M. Goldys, I.L. Guy , Appl. Phys. Lett., vol. 75 , p. 3965, 1999.

[7] B.J. Rodriguez, D.J. Kim, A.I. Kington, R. Nemanich, in Mater. Res. Soc. Symp. Proc., vol. 693, 2001, pp. I91-9.

[8] Iborra, J. Olivares, M. Clement, L. Vergara,A. Sanz-Hervas, J. Sangrador, "Sensors and Actuator A," vol. 115, pp. 501-507, 2004.

[9] J. Olivares, M. Clement, M. Iborra, E. Vergara, L. Sánchez-Rojas, J.L. Vázquez, P. Sanz, in Proc. SPIE Smart Sensors, Actuators, and MEMS II , Sevilla,Spain, 2005, pp. 16-26.

[10] D. Kapolnek, S. Keller, R. Vetury, R.D. Underwood, P. Kozodoy, S.P. DenBaars, U.K. Mishra, Appl.Phys. Lett, vol. 71 , pp. 1204-1206, 1997.

[11] Y. Kwaguchi, G. Sugahara, A. Mochida, T. Shimamoto, A. Ishibashi, Y. Yokogawa, Phys. Status Solidi C, vol. 0, pp. 2107-2110, 2003.

[12] T. Katona, P. Cantu, S. Keller, Y. Wu, J. Speck, S. DenBaars, Appl. Phys. Lett., vol. 84, pp. 5025-5027, 2004.

[13] S. Tanaka, Y. Kawaguchi, K. Yamada, N. Sawaki, M. Hibino, K. Hiramatsu, in Proc. International Workshop on Nitride Semiconductors, Nagoya, Japan, 2000, pp. 300-3.

[14] T. Asano, K. Yanashima, T. Asatsuma, T. Hino, T. Yamaguchi, S. Tomiya, K. Funato, T. Kobayashi, M. Ikeda, Phys. Status Solidi A, vol. 176, pp. 23-30, 1999.

[15] S. Heikman, S. Keller, S.P. Den Baars, U.K. Mishra, F. Bertram, J. Christen, Jpn. J.Appl. Phys. Part 1, vol. 42, pp. 6276-83, 2003.

[16] S. Hara, S. Motoaki, S. Kuypers Jan Abe, S. Takashi, S.E. Masayoshi, Sensors Actuators A , vol. 117 (2), p. 211-216, 2005.

[17] M. Sarzynski, M. Krysko, G. Targowski, G. Kamler, J. Domagalstroka, R. Czernecki, A. Libura A, P. Perlin, M. Leszczynski,, Appl. Phys. Lett., vol. 91, pp. 221103-1-3, 2007. 
[18] W. Changju, J. Zhonghe, W. HuiQuan, M. Huilian, W. Yuelin , J. Micromech. Microeng., vol. 17 , pp. 2393-2397, 2007.

[19] E. Saucedo-Flores, R. Ruelas,M. Flores, Jung-Chih Chiao, "Study of the pull-in voltage for MEMS parallel plate capacitor actuators," Mater. Res. Soc., vol. 782, pp. 355-61, 2004.

[20] G. M. Rebeiz, RF MEMS Theory, Design and Technology. 1st Edition, John Wiley \& Sons, Inc., 2003.

[21] R. Mahameed, N. Sinha, M.B. Pisani, G. Piazza, "Dual-beam actuation of piezoelectric AIN RF MEMS switches monolithically integrated with AlN contourmode resonators," Journal of Micromechanics and Microengineering, vol. 18, no. 10, p. 105011, 2008.

[22] G. T. Z. SKI. [Online]. http://www.ippt.gov.pl/ tzielins/doc/stec_tgzielinski_Piezo.paper.pdf

[23] J. D. Leo, "Engineering Analysis of Smart Material Systems," in Engineering Analysis of Smart Material Systems. Hoboken, New Jersey/ USA: John Wiley \& Sons, 2007, ch. 4, p. 136.

[24] R.C. Turner, P.A. Fuierer, R.E. Newnham, T.R. Shrout, Appl. Acoust. , vol. 41, p. 299, 1994.

[25] K. Tonisch, V. Cimalla, F. Niebelsch utz, H. Romanus, M. Eickhoff, O. Ambacher, in Mater. Res. Soc. Symp.Proc. , vol. 955, 2006, pp. I16-03.

[26] Y.T. Yang, K.L. Ekinci, X.M.H. Huang, L.M. Schiavone, M.L. Roukes , Appl. Phys. Lett., vol. 78 , p. 162.

[27] Y. Kuo, W. Lin, Jpn. J. Appl. Phys., vol. 41, p. 5557-5558, 2002.

[28] J.L. Weyher, P.D. Brown, J.L. Rouviere, T. Wosinski, A.R.A. Zauner, I. Grzegory, J. Cryst. Growth, vol. 210, p. 151, 2000.

[29] D. Zhuang, J.H. Edgar, J. Liu, B. Liu, L. Walker, MRS Internet J. Nitride Semicond. Res. , vol. 7, p. 4, 2002.

[30] I. Cimalla, Ch. Forster, V. Cimalla, V. Lebedev, D. Cengher, O. Ambacher, Phys. Status Solidi c, vol. 3, p. 1767, 2006.

[31] T.Y. Sheng, Z.Q. Yu, G.J. Collins, Appl. Phys. Lett., vol. 52, p. 576, 1988.

[32] H.M. Ng, W. Parz, N.G. Weimann, W. Chowdhury, Jpn. J. Appl. Phys, vol. Part 2 42, p. L1405, 2003.

[33] I.M. Tiginyanu, V. Popa, O. Volciuc, Appl. Phys. Lett., vol. 86, p. 174102, 2005.

[34] Gao Y 2004 PhD Thesis University of California, Santa Barbara.

[35] Ebbers A 2003 PhD Thesis Ruhr-Universit“at Bochum

[36] A.R. Stonas, N.C. MacDonald, K.L. TurnerL, S.P. DenBaars, E.1. Hu, J. Vac. Sci. Technol. B , vol. 19, p. 2838, 2001.

[37] R.P. Strittmatter, R.A. Beach, T.C. McGill, Appl. Phys.Lett., vol. 78 , p. 3226, 2001.

[38] M. S. Minsky, M. White, and E. L. Hu, Appl. Phys. Lett. , vol. 68, p. 1531, 1996.

[39] D. Marx, Z. Kawazu, T. Nakayama, Y. Mihashi,T. Takami, M. Nunoshita, T. 
Ozeki, Journal of Crystal Growth, vol. 180-190, pp. 87-91, 1998.

[40] T. Asano, K. Yanashima, T. Asatsuma, T. Hino, T. Yamaguchi,S. Tomiya, K. Funato2), T. Kobayashi, M. Ikeda, phys. stat. sol. (a), vol. 176, no. 23, pp. 23-29, 1999.

[41] H.Harima, "Properties of $\mathrm{GaN}$ and related compounds studied by means of Raman scattering," J. Phys.: Condens. Matter, vol. 14, p. R967-R993, 2002.

[42] R. Liu and F. A. Ponce, A. Dadgar and A. Krost, Appl.Phys, Lett., vol. 85, no. 5, pp. 860-862, 2003.

[43] V. Lebedev, B. Schr. oter, G. Kipshidze, Wo. Richter, J.Crystal Growth, vol. 207, p. 266, 1999.

[44] A.T. Schremer, J.A. Smart, Y. Wang, O. Ambacher, N.C. MacDonald, J.R. Shealy, Appl. Phys. Lett., vol. 76, p. 736, 2000.

[45] [Online]. www2.angstrom.uu.se/.../m raman.html

[46] Harris , Bertolucci, Symmetry and Spectroscopy. Dover Publications. , 1989.

[47] [Online]. http://newton.ex.ac.uk/research/biomedical/optics/images/Raman.jpg

[48] D. Willock, Molecular Symmetry. UK: John Wiley and Sons, 2009.

[49] [Online]. http://www.kosi.com/Raman_Spectroscopy/rtr-ramantutorial.php?ss=800

[50] D. W. Hahn. [Online]. http://plaza.ufl.edu/dwhahn/Raman\%20Scattering\%20Theory.pdf

[51] M. J. Pelletier, Analytical Applications of Raman Spectroscopy. Wiley-Blackwell, 1999.

[52] John R. Ferraro, Kazuo Nakamoto, Chris W. Brown, Introductory Raman spectroscopy. Academic Press, 2003.

[53] http://www.tutornext.com/system/files/u83/UNITED\%201.GIF.

[54] F. Campbell, Elements of metallurgy and engineering alloys. ASM International, 2008.

[55] K. Okamoto, Fundamentals of optical waveguides. Gulf Professional Publishing, 2000 .

[56] J. Singh. . [Online]. http://www.eecs.umich.edu/ singh/

[57] Ian R. Lewis, Howell G. M. Edwards, Handbook of Raman spectroscopy:from the research laboratory to the process line. CRC press, 2001.

[58] [Online]. http://www.scielo.br/img/revistas/ce/v50n315/a11fig01.gif

[59] M, Cardona;G G“untherodt, "Light Scattering in Solids II," Springer Topics in Applied Physics, vol. 50, p. 19-178, 1982.

[60] I. Gorczyca, N. E. Christensen, E. L. Peltzer y Blancá, and C. O. Rodriguez, "Optical phonon modes in GaN and AlN," Phys. Rev. B , vol. 51, p. 11936-11939, 1995.

[61] C. Kittel, Introduction to Solid State Physics, 7th ed. John Wiley and Sons, 1996.

[62] L.E. Rodak, Sridhar Kuchibhatla and D. Korakakis, Mater. Lett., vol. 63 , pp. 15711573, 2009.

[63] E.F. McCullen, J.S. Thakur, Y.V. Danylyuk, G.W. Auner, L.W. Rosenberger, J. 
Appl. Phys., vol. 103, pp. 063504-063504-4, 2008.

[64] J. R. Mileham, S. J. Pearton, C. R. Abernathy1, J. D. MacKenzie, R. J. Shul, and S. P. Kilcoyne, "Wet chemical etching AlN ," Apl.Phys.Lett, vol. 67, no. 8, pp. 11191121, 1995.

[65] K. Jagannadham, A. K. Sharma, Q. Wei, R. Kalyanraman, and J. Narayan, "Structural characteristics of AlN films deposited by pulsed laser deposition and reactive magnetron sputtering: A comparative study," J. Vac. Sci. Technol. A, vol. 16, p. 2804, 1998.

[66] L. Bergman, D. Alexson, R. J. Nemanich, M. Dutta, M. A. Stroscio, C.Balkas, and R. F. Davis, MRS Internet J. Nitride Semicond.Res., vol. 4S1, p. G665, 1999.

[67] M. Beshkova, Z. Zakhariev, M. V. Abrashev, E. Birch, A. Kakanakova and R. Yakimov, Vacuum, vol. 76, p. 143, 2004.

[68] M. Bickermann, B. M. Epelbaum, P. Heimann, Z. G. Herro, and A. Winnacker, Appl. Phys. Lett., vol. 86, p. 13190, 2005.

[69] T. Prokofyeva, M. Seon, J. Vanbuskirk, M. Holtz, S. A. Nikishin, N. N.Faleev, H. Temkin, and S. Zollner, Phys. Rev. B, vol. 63, p. 125313, 2001.

[70] A. Sanz-Hervás, E. Iborra, M. Clement, J. Sangrador, and M. Aguilar,, Diamond Relat. Mater. , vol. 12, p. 1186, 2003.

[71] M. F. MacMillan, R. P. Devaty, and W. J. Choyke, Appl. Phys. Lett., vol. 62, p. $750,1993$.

[72] J. Ibanez, S. Hernandez, E. Alarcon-Llado, R. Cusco, L. Artus, S.V. Novikov, C.T. Foxon, E. Calleja, J. Appl. Phys., vol. 104, p. 033544, 2008.

[73] M. Dean Sciacca, A. J. Mayur, Eunsoon Oh, A. K. Ramdas, S. Rodriguez,J. K. Furdyna,M. R. Melloch,C. P. Beetz and W. S. Yoo, "Phys. Rev. B ," vol. 51, p. 7744-7752, 1995.

[74] F. Kaneko et al., Spectrochimica Acta, vol. Part A 60 , p. 9-18, 2004.

[75] B. Aspar, R. Berjoan, B. Armas, D. Perarnau, J. Phy., vol. 3, p. 171, 1993.

[76] M. Kazan, R. Nader, E. Moussaed, P. Masri,, J. Cryst. Growth, vol. 290, p. 44, 2006.

[77] A.R. Chowdhuri, C.G. Takoudis, R.F. Klie, N.D. Browning, Appl. Phys. Lett, vol. 80, p. 4241, 2002.

[78] V. Lebedev, U. Kaiser, C. Foerster, J. Pezoldt, J. Biskupek, O. Ambacher, J. Appl. Phys., vol. 97, p. 114306.

[79] E. Iborra, M. Clement, L. Vergara, A. Sanz-Hervas, J. Olivares, J. Sangrador, Appl.Phys. Lett., vol. 88, p. 231901, 2006).

[80] T. Hanada, M. Kobayashi, S. Tanabe, N. Soga, J. Non-Cryst. Solids, vol. 135, p. 227, 1991.

[81] A. Jacquot, B. Lenoir, A. Dauscher, P. Verardi, F. Craciun, M. Stolzer, M. Gartner, M.Dinescu, Appl. Surf. Sci., vol. 186, p. 5071, 2002.

[82] X. Hao, M. Yu, D. Cui, X. Xu, Y. Bai, Q. Wang, M. Jiang, J. Cryst. Growth, vol. 242, p. 229, 2002.

[83] S. Kuchibhatla, L.E. Rodak, D. Korakakis , Thin Solid Films, vol. 519, no. 1, pp. 
117-121.

[84] L. Bergman, M. Dutta, C. Balkas, R .F. Davis, J. A. Christman, D. Alexson, R. J. Nemanich, " J. Appl. Phys. ," vol. 85 , p. 3535, 1999.

[85] M. Bickermann, B. M. Epelbaum, A. Winnacker, phys. stat. sol. (c) 0 , vol. 7, p. 1993-1996, 2003.

[86] Vanni Lughia, David R. Clarke, Apl.Phys.Lett, vol. 89, p. 241911, 2006.

[87] K. Kuball, J. M. Hayes, Ying Shi , J. H. Edgar, Appl. Phys. Lett., vol. 77, pp. 19582000.

[88] P. Perlin, A. Polian, and T. Suski, Phys. Rev.B , vol. 47, pp. 2874-1993.

[89] Alejandro B. Rodriguez-Navarro, Thin Solid Films, vol. 389, pp. 288-295, 2001.

[90] S. Fukumoto, T.Hookabe, H. Tsubakino, J. Mater. Sci, vol. 35, p. 2743-2748, 2000.

[91] [Online]. http://science.jrank.org/pages/271/Aluminum-Hydroxide-Properties.html

[92] M. Kishi, M. Suzuki, K. Ogawa, Jpn.J.Appl.Phys., vol. 31, no. 4, pp. 1153-1159, 1992.

[93] M. J. Madou, Fundamentals of microfabrication:the science of miniaturization. CRC Press, 2002.

[94] S. D. Senturia, Microsystem Design. Kluwer academic , 2001.

[95] W. M. Vim and R. J. Paff, J.Apl.Phys, vol. 45, no. 3, pp. 1456-1457, 1974.

[96] K. WANG and R. R. REEBER, in Mater. Res. Soc. Symp. Proc., vol. 482 , 1998, p. 863.

[97] J. M. Hayes, M. Kuball, Y. Shi, and J. H. Edgar, Jpn.J.Apl.Phys, vol. 39, p. L710, 2000.

[98] A. Alex .V olinskya, B.V ellaa, W.Gerberich, Thin Solid Films, vol. 429, p. 201$210,2003$.

[99] piezoelectric . [Online]. http://upload.wikimedia.org/math/4/8/0/4807f29209b29d3d30349f13f7f7a6f4.png

[100] G. S. MATHON, "PIEZOELECTRIC ALUMINUM NITRIDE," UNIVERSIDAD DE LA REPÚBLICA ORIENTAL DEL URUGUAY Dissertation, 2009.

[101] L. Chen, J-H.. Li, J. Slutsker, J. Ouyang, and A.L. Roytburda, J. Mater. Res., vol. 19, no. 10, 2004.

[102] R. Herdier, D. Jenkins, E. Dogheche, D. Remiens, M. Sulc , Rev. Sci. Instrum., vol. 77, no. 9, pp. 93905-1-5, 2006.

[103] W. Cao, S. Zhu, B. Jiang, J. Appl. Phys., vol. 83, no. 8, pp. 4415-20, 1998.

[104] D. Chen, J. Wang, D. Li, L. Zhang, X. Wang, Appl Phys A, vol. 100, p. 239-244, 2010.

[105] M. Clement, J. Olivares, E. Iborra, S. González-Castilla, N. Rimme, A. Rastogi, Thin Solid Films , vol. 517, p. 4673-4678, 2009.

[106] A. Artieda, C. Sandu, P. Muralt, J. Vac. Sci. Technol. A, vol. 28, no. 3, 2010.

[107] Shear mode actuated valve. [Online]. http://www.dimatix.com/technology/dimatixpiezoelectric.asp 
[108] J. R. Mileham, S. J. Pearton,a) C. R. Abernathy, J. D. MacKenzie, R. J. Shul and S. P. Kilcoyne, "Wet chemical etching of AlN," Appl. Phys. Lett., vol. 67, no. 8, 1995. [109] e-funda. [Online]. http://www.efunda.com/formulae/solid_mechanics/mat_mechanics/strain.cfm 


\section{Appendix}

Program to calculate the peak height from laser Doppler vibrometer data.

clear all;

clc;

$\mathrm{c}=$ input('Enter frequency of operation: ');

v=input('Enter Voltage of operation : ');

$\%$ Change the space after $\mathrm{kHz}$ just in case if the filename doesn't match.

textfilename $=$ ['img 05 200um 20V sine ' num2str(c) 'kHz ' num2str(v) 'V.txt']

data=dlmread(textfilename,'ไt',5,0);

freq=data $(:, 1)$;

displ=data $(:, 2)$;

$\mathrm{f} 1=\mathrm{c} * 1000$

$\mathrm{f} 2=2 * \mathrm{f} 1$

$[\mathrm{r} c]=\operatorname{size}($ data $)$

for $\mathrm{i}=1$ :length(freq)

if $(\operatorname{freq}(\mathrm{i})==\mathrm{f} 1)$

index $1=\mathrm{i}$;

end

if $($ freq $(i)==f 2)$

index $2=\mathrm{i}$

end

end

disp(['Peak height at ' num2str(f1) 'Hz, ' num2str(v) 'V']);

$\mathrm{y} 1=\operatorname{displ}($ index 1$)$-displ(index $1+4)$

disp(['Peak height at ' num2str(f2) 'Hz, ' num2str(v) 'V']);

$\mathrm{y} 2=\operatorname{displ}($ index 2$)-\operatorname{displ}($ index $2+4)$ 